\title{
Angst im Zusammenhang mit Schwangerschaft und Geburt
}

\author{
Wissenschaftliche Arbeit zur Erlangung \\ des Grades einer Diplom-Psychologin \\ im Fachbereich Psychologie \\ der Universität Konstanz
}

vorgelegt von

Katrin Oberndörfer

Matr.Nr. 01/406754

Erstgutachterin: Professorin Dr. Brigitte Rockstroh

Zweitgutachter: Professor Dr. Wilhelm Kempf

Kreuzlingen, im April 2003 


\section{Mein Dank...}

...gilt allen Personen, die mit ihrer fachlichen und persönlichen Unterstützung zum Gelingen dieser Arbeit beigetragen haben:

Prof. Dr. Brigitte Rockstroh für die Betreuung meiner Arbeit sowie für das Interesse und die Aufgeschlossenheit für ein „Schwangerschafts-/Geburtsthema“.

Dr. Willi Nagl für sein unendliches Engagement und die Geduld, die er mir bei der Hilfe meiner Statistik-Probleme entgegengebrachte.

Prof. Dr. Wilhelm Kempf, der sich bereit erklärte den Posten des Zweitbetreuers zu übernehmen sowie für seine Hinweise bei der Fertigstellung meiner Arbeit.

Den Hebammen Maria Canders, Sabine Friese-Berg, Petra Harder, Rita Mosel, Elke Schlupf, Christiane Schnur, Heidi Ullmann und Michaela Zunftmeister, die mir eine Untersuchung in ihren Praxen bzw. Kursen ermöglicht haben und deren Unterstützung bei der Durchführung.

Dr. med. A. Mosel, der mich in meinem Vorhaben unterstützte und mir die Durchführung der Untersuchung am Krankenhaus-Spitalfond Waldshut-Tiengen erleichterte.

Den Teilnehmerinnen dieser Untersuchung, die sich die Zeit nahmen, die Fragebögen auszufüllen.

Markus Läubli, der mich mit viel Geduld und Tatkraft bei "Computerangelegenheiten" unterstützte sowie für den emotionalen Beistand.

Birgit Räuber, Ann Rottmann, Marina Uhl und Uli Wolf, die meine Arbeit Korrektur lasen und mir wichtige Anregungen gaben.

Dem Retter in der Not, Johann Gessler, dem es gelang, mein „Word“ am 5. April 2003, nach völligem Zusammenbruch wieder funktionstüchtig zu machen.

Meinen Kolleginnen, insbesondere Gertrud Wolf, die mir immer den nötigen Freiraum für mein Studium gewährten.

Mein Dank gilt auch meinen Eltern, die mich während meines Studiums unterstützten und immer an mich glaubten. 


\section{Inhaltsverzeichnis}

1 EINLEITUNG.

\section{THEORIE.}

$2.1 \quad$ Angst 3

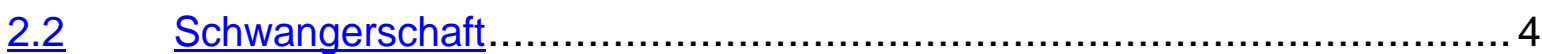

2.2.1 Schwangerschaft als Krise/kritisches Lebensereignis ........................ 6

2.2.2 Risikoschwangerschaft/Schwangerschaftskomplikationen ..................

2.2.3 Schwangerschaftskomplikationen und Angst.................................. 13

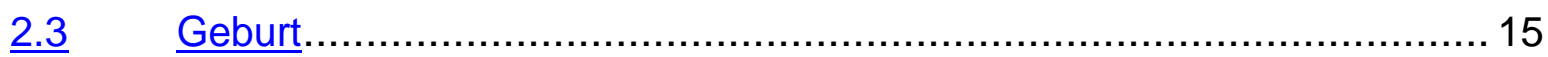

2.3.1 Physiologie der Geburt ....................................................... 16

2.3.2 Pathologie der Geburt/Geburtskomplikationen ............................. 18

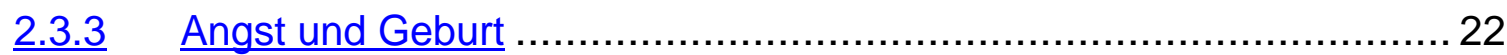

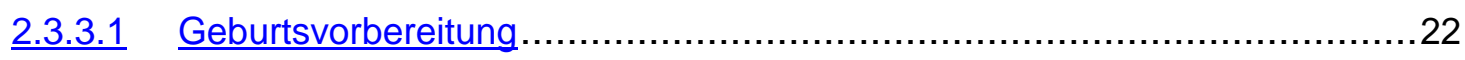

2.3.3.2 Psychische Korrelate des Geburtsverlaufs........................................26

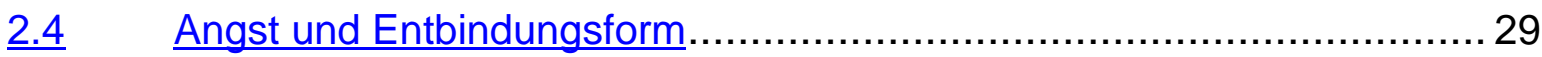

2.5 Erklärungsmechanismen für die Wirkung von Angst auf den

Schwangerschafts- und Geburtsverlauf .......................................... 34

2.6 Fragestellungen und Hypothesen ……........................................ 36

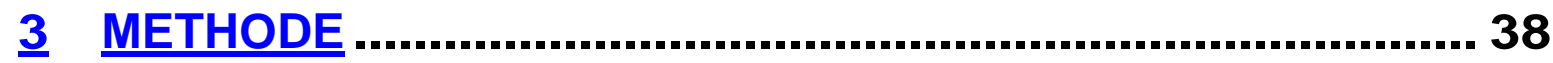

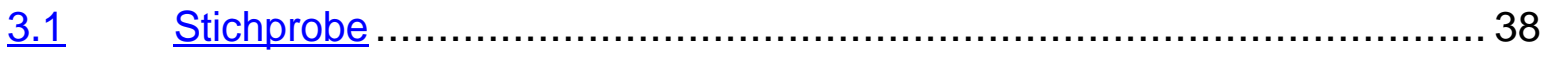

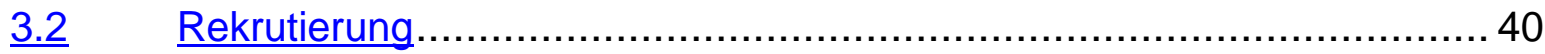

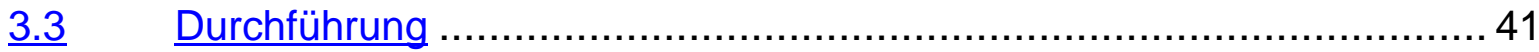


3.4 Verwendete Verfahren und Variablenbildung................................... 43

3.4.1 Soziodemographische und schwangerschaftsspezifische Fragen......... 43

3.4.2 Fragebogen zur Erfassung der Angst............................................ 43

3.4.3 Erfassung von Komplikationen .................................................. 45

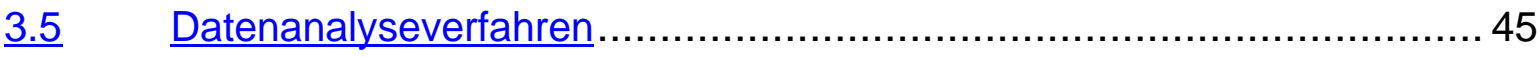

3.5.1 Berechnung von Gruppenunterschieden ……............................. 46

3.5.2 Weitere durchgeführte Untersuchungen (explorativer Art)................ 47

\section{ERGEBNISSE}

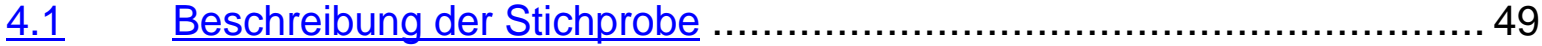

4.1.1 Soziodemographische und schwangerschaftsspezifische Daten .......... 49

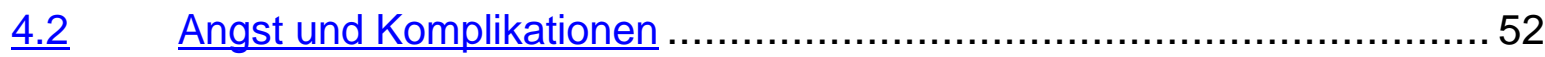

4.2.1 Zusammenhang von Angst und Schwangerschaftskomplikationen ...... 52

4.2.2 Zusammenhang von Angst und Geburtskomplikationen....................52

4.3 Zusammenhang zwischen Komplikationen und Entbindungsform...........55

4.4 Zusammenhang zwischen Angst und Entbindungsform...................... 57

4.5 Weitere durchgeführte Untersuchungen …....................................59

4.5.1 State- und Trait-Angst (unabhängig von Entbindungsform) ............... 59

4.5.1.1 State-Angst (abhängig und unabhängig von Parität)................................59

4.5.1.2 Trait-Angst (abhängig und unabhängig von Parität) …...........................61

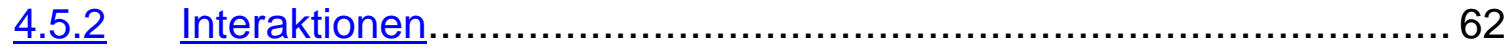

4.5.3 Bestimmung von Prädiktoren......................................................67

4.6 Zusammenfassung der Ergebnisse …......................................... 72

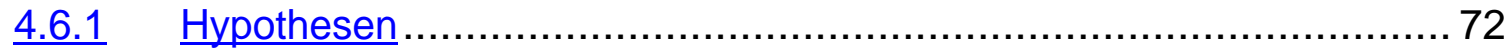

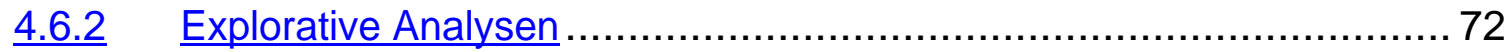

4.6.2.1 State- und Trait-Angst (unabhängig von der Entbindungsform) ................72

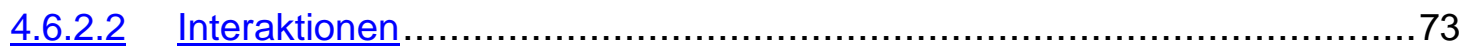

4.6.2.3 Bestimmung von Prädiktoren .......................................................73 
$\underline{5}$ DISKUSSION

5.1 Diskussion der Ergebnisse …............................................... 75

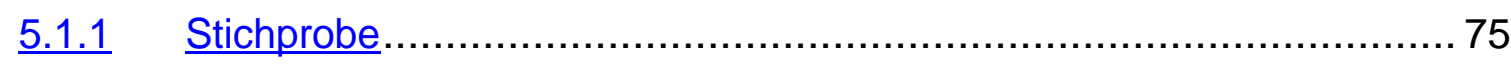

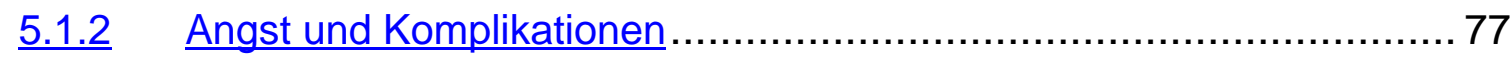

5.1.3 Komplikationen und Entbindungsform ……................................ 79

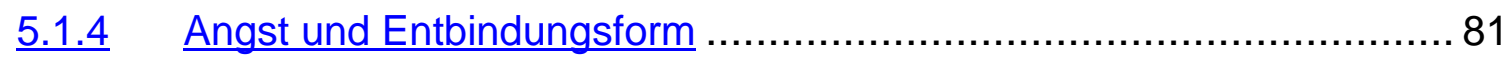

5.1.5 Weitere durchgeführte Untersuchungen ………......................... 82

5.1.5.1 State- und Trait-Angst unabhängig von der Gruppenzugehörigkeit nach Entbindungsform (abhängig und unabhängig von der Parität)....................82

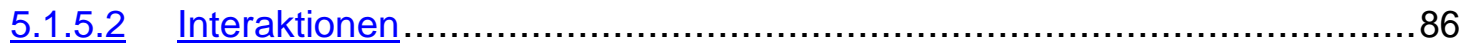

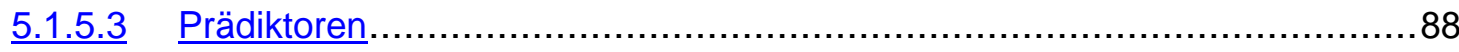

5.2 Stärken und Schwächen der Untersuchung ..................................... 90

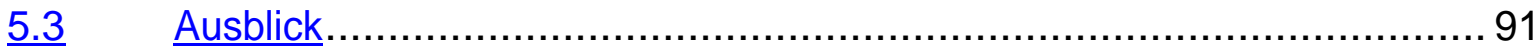

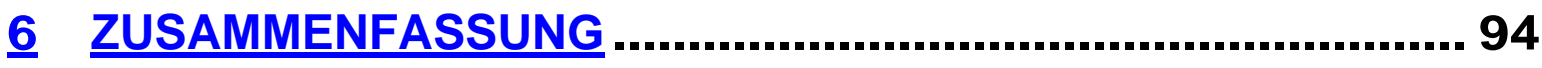

LITERATURVERZEICHNIS....................................................... 96

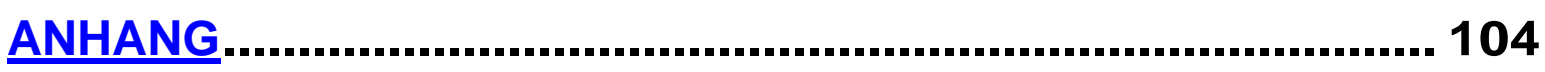

Anhang A: Explorative Analysen ...................................................... 105

Anhang B: $\quad$ Verwendeter Fragebogen ................................................. 110 


\section{Einleitung}

Untersuchungen zu dem Thema „Zusammenhang zwischen Angst und Komplikationen in der Schwangerschaft und während des Geburtsverlaufs" zeigten überwiegend, dass Frauen die eher ängstlich sind, zu komplikationsreicheren Schwangerschaften und Geburtsverläufen neigen, während dies bei Frauen, die weniger ängstlich sind, seltener der Fall ist. Bisher wurde jedoch meist nicht der Verlauf von Angst gemessen, sondern die Erhebung von Angst fand nur zu einem Zeitpunkt statt (Crandon, 1979). Des weiteren erhoben nur wenige Forscher Angst auch nach der Geburt (Rizzardo, Magni, Cremonese, Talamo Rossi \& Cosentino, 1988).

Der Zusammenhang zwischen Angst und Komplikationen in Abhängigkeit der Entbindungsform wurde bei bisherigen Forschungsarbeiten weitgehend vernachlässigt. Ergebnisse für Deutschland liegen nur von Windsor-Oettel (1992) vor.

Daher soll in der vorliegenden Arbeit der Zusammenhang zwischen Angst und Komplikationen während der Schwangerschaft und der Geburt prospektiv sowie der Verlauf von Angst (präpartal und postpartal) untersucht werden. Ausserdem wird die Entbindungsform berücksichtigt.

Bei der gemessenen Angst handelt es sich um allgemeine Angst (im Gegensatz zu situationsspezifischer Angst), gemäss dem Trait-State-Angstmodell von Spielberger (1972). Es werden solche Komplikationen erfasst, die in einschlägigen Fachbüchern beschrieben werden (z.B. Martius, 1990) und die gemäss der Gesellschaft für Qualität in der ausserklinischen Geburtshilfe e.V. (Stand 2001) erfasst werden sollten.

Die Untersuchung erfolgte bei Frauen, die an einem hebammengeleiteten Geburtsvorbereitungskurs teilnahmen und entweder eine Hausgeburt oder eine Klinikgeburt anstrebten. 
Ziel ist, den Zusammenhang zwischen Angst und Komplikationen zu präzisieren. Sollte sich ein Zusammenhang zeigen, könnte dies als ein weiterer Hinweis für aus der Literatur bekannte Befunde angesehen werden (McDonald,1968; Gorsuch \& Key, 1974, Rizzardo et al., 1988). Ausserdem würde dies die Wichtigkeit angstreduzierender Arbeit von Personen, die rund um die Geburt und Schwangerschaft tätig sind, unterstrichen werden.

Sollten sich beim Vergleich Unterschiede zwischen den Gruppen bezüglich der Angst darstellen, so könnte dies ein erster Hinweis dafür sein, dass sich Klinik- und Hausgeburtsfrauen bezüglich allgemeiner Ängstlichkeit unterscheiden. 


\section{Theorie}

Im Folgenden werden grundlegende theoretische Erkenntnisse von Angst, Schwangerschaft, Geburt und Entbindungsformen dargestellt sowie ein Überblick der bestehenden Forschung zu diesen Themen gegeben.

\subsection{Angst}

\section{Persönlichkeitsmerkmal und emotionaler Zustand}

Aktuelle Ansätze verstehen die Angstemotion „als ein mit bestimmten Situationsveränderungen intraindividuell (innerhalb eines Individuums) variierender affektiver Zustand (state) des Organismus, der durch spezifische Ausprägungen auf physiologischen, verhaltensmässig-expressiven und subjektiven Parametern gekennzeichnet ist“ (Krohne, 1996, S. 5). Nach Spielberger (1972) ist Angst ein Zustand, der durch eine Erhöhung der Aktivität des autonomen Nervensystems sowie durch selbstwahrgenommene Erregung, des Gefühls von Anspannung und Bedrohung sowie durch erhöhte Besorgnis charakterisiert wird.

Angst als habituelles Persönlichkeitsmerkmal wird für gewöhnlich mit dem Begriff "Ängstlichkeit“ gleichgesetzt. Ängstlichkeit benennt die intraindividuell verhältnismäßig dauerhafte, aber interindividuell abweichende Tendenz (trait), Situationen als Bedrohung wahrzunehmen und darauf mit einem höheren Angstzustand zu antworten. Folglich empfinden manche (ängstliche) Menschen bestimmte Situationen (z.B. bevorstehende Fahrprüfung) im allgemeinen als beunruhigender und reagieren dementsprechend mit einem höheren Anstieg der Angst als andere (nichtängstliche) Menschen (Krohne, 1996). Hochängstliche neigen auch dazu, mehr Situationen als Bedrohung anzusehen (Laux et al., 1981).

Angst lässt sich auf drei verschiedenen Ebenen operationalisieren:

B Subjektiv-verbale Selbstbeschreibung

B Physiologische Reaktionen

B Ängstliches Verhalten (z.B. Mimik) 
Zu den physiologisch messbaren Reaktionen zählen z.B. Erhöhung der Herzfrequenz, des Blutdrucks, der Schweissekretion und des Muskeltonus sowie hormonelle Änderungen wie z.B. der Anstieg von Adrenalin und Noradrenalin (Pinel, 2002; Sartory, 1997; Barlow, 1988; Larbig \& Birbaumer, 1986). Bei sehr starken Angstzuständen kann es infolge Atemproblemen zu einer respiratorischen Alkalose mit Abfall des $\mathrm{CO}_{2}$-Partialdrucks kommen (Barlow, 1988).

Auf der Ebene der Physiologie und des Verhaltens präsentiert Ängstlichkeit eine äusserst hohe Individualspezifität der Reaktionen (Asendorpf, 1996; Levitt, 1971).

\subsection{Schwangerschaft}

Zuvor soll ein Exkurs zur Familienentwicklung dem besseren Verständnis der anschliessend beleuchteten Aspekte von Schwangerschaft dienen.

\section{Historische Entwicklung der Familie in unserem Kulturkreis}

In allen Kulturen gilt die Fruchtbarkeit als ein hohes Gut und grundlegende Bedingung für die Gemeinschaft. Im Kinderwunsch treffen persönliche und gesellschaftliche Bedürfnisse zusammen.

Unser heutiges Wort "Familie“ als Benennung einer anverwandten Personengruppe, die in einem gemeinsamen Haushalt zusammenlebt, besteht erst seit dem 18. Jahrhundert. Der Name lässt sich aus dem lateinischen Wort „familia“ ableiten, welches die Bedeutung „Hausgenossenschaft" hat. Vom Mittelalter an bis zur Neuzeit war die Familie eine Gemeinschaft aller im Haus zusammenlebender Menschen inklusive des Gesindes. Kinder und Gesinde hatten einen ähnlichen Stellenwert. Das Zusammenleben war von wirtschaftlichen Überlegungen bestimmt. Der Nachwuchs war zum Überleben zwingend. Vertrautheit und Emotionalität hatten in dieser Familienform eine viel geringere Bedeutung als heutzutage (Brähler, 1990). Bis zum 17. Jahrhundert wurde Kindern von den Familien wenig Beachtung geschenkt. Mutterliebe hatte kein hohes Ansehen, und die Kindersterblichkeit war äusserst hoch. Das Aussetzen von Kindern diente der 
Regulation der Familiengrösse und wurde von der Gesellschaft nicht geächtet (Badinter, 1980).

Ab dem 17. Jahrhundert kam es aufgrund der Industrialisierung zu einem elementaren Wandel der Familienform. Da Wohn- und Arbeitsort getrennt wurden, verlor die Familie ihre Bestimmung als Produktionsgemeinschaft und so kam es zur Privatisierung der Familie. Die Beziehungen innerhalb der Familienmitglieder wurden tiefer und emotionaler (Brähler, 1990).

Ende des 18. Jahrhunderts kam es zu bahnbrechenden Einstellungsänderungen im Umgang mit Kindern. Das Kind wurde zum König und zum kostbarsten Vermögen der Familie, die elterliche Autorität wurde jetzt mit dem Wohl des Kindes gerechtfertigt (Badinter, 1980). Der Veränderungsprozess im Erziehungswesen führte zur Einführung der allgemeine Schulpflicht (Brähler, 1990). Aus der Familie war ein Hort der Privatheit und Verbundenheit geworden, der einen gefühlsmäßigen Ausgleich zur Arbeitswelt des Mannes bringen sollte. Die Mutterrolle wurde im Leben einer Frau zentral und ihr wurde Ehre und Hochachtung entgegengebracht (Badinter, 1980).

Diese Polarisierung von Wohn- und Produktionsbereich und die daraus folgende Arbeitsteilung zwischen Mann und Frau war die Basis zur Ausbildung der Geschlechtscharaktere: die Frau als Hüterin des Lebens, wobei die Hausarbeit und die Kindererziehung als Liebesdienst verstanden wurden, der Mann als Ernährer der Familie, sich im Berufsleben zu behaupten hatte (Berninghausen, 1980).

Die Untersuchungen zur gegenwärtigen Familiensituation lassen deutlich erkennen, dass es keine klare Begriffsbestimmung der Geschlechtsrollen mehr gibt. Die Familie kann nicht mehr in allgemeingültigen Termini beschrieben werden, da durch die Verknüpfung alter Geschlechtscharaktere mit neuen Verhaltensmöglichkeiten eine Vielzahl an Rollen entstehen. Als allgemeiner Tenor gilt die Angleichung der Geschlechterrollen. Dabei werden Männer tendenziell mütterlicher (Badinter, 1980; Beckmann, 1979). Durch den Aufgabenverlust der Väter werden sie aber auch entlastet, was die Chance auf neue 
Verhaltensmöglichkeiten mit sich bringt. Dieser Umstrukturierungsprozess innerhalb der Familie kann Verunsicherungen aufkommen lassen, welche diffuse Ängste auslösen können (Brähler, 1990).

\section{Beweggründe für eine Mutter-/Vaterschaft}

Berninghausen (1980) gibt einen Überblick über bewusste Motive der Frauen für Schwangerschaft, die sie aus verschiedenen Studien zusammengefasst hat:

B Wünsche nach dem Erleben der körperlichen Veränderung in der Schwangerschaft und Hoffen auf Selbstverwirklichung

B Konsolidierung der Partnerschaft durch die Vereinigung in einem Kind und durch die gemeinsame Aufgabe

B Verbesserung der Beziehung zu den eigenen Eltern und Beweis der eigenen Reife (Erwachsensein) erbringen

B Sinngebung und befriedigende Aufgabe

B Durch das Kind ein Stück vom Mann besitzen

B Altersversorgung durch das Kind und die Vorstellung im Kinde weiterzuleben

B Drang zur Erweiterung von Erfahrungsbereichen (durch das Leben mit dem Kind)

B Normative Gesichtspunkte

\subsubsection{Schwangerschaft als Krise/kritisches Lebensereignis}

In der Literatur wird Schwangerschaft schon sehr lange als Krisenzeit unter dem Gesichtspunkt vom Beziehungswechsel der Dyade zur Triade erörtert (Hill, 1949; LeMasters, 1957; Erikson, 1966; Wimmer-Puchinger, 1992). Auch Baltes (1990) sieht Schwangerschaft als Entwicklungskrise.

Ausgehend von einer die gesamte Lebensspanne umfassende Entwicklungspsychologie ist die Geburt eines Kindes, vor allem die des ersten, als ein wichtiger Entwicklungsprozess im frühen Erwachsenenalter zu betrachten, mit weitreichenden Auswirkungen auf den weiteren Lebenslauf und der Persönlichkeitsentwicklung (Baltes, 1990). 
Weniger negativ ist die alternative Bezeichnung „normative Krise“ (Rapaport 1963, zit. nach Wimmer-Puchinger, 1992). Schwangerschaft bedeutet für die werdenden Eltern eine Neuorientierung der Persönlichkeit und des bisherigen Alltagslebens. Jedoch unterscheidet sich dieser normative Übergang im menschlichen Lebenslauf von anderen kritischen Lebensereignissen wie z.B. Tod des Partners, schwere Krankheit. Elternschaft muss nicht immer einen belastenden Charakter annehmen, sie kann auch als positiver Entwicklungsimpuls erlebt werden (Wimmer-Puchinger, 1992).

Nach Guttman (1975) haben Krisen sowohl eine objektive als auch subjektive Dimension, das heisst, dass was für den einen Menschen eine Schicksalsschlag ist, für den anderen durchaus leicht zu bewältigen ist. Verschiedene Untersuchungen zum Thema Elternschaft unter dem Krisenaspekt haben gezeigt, dass das Erleben der Geburt als eine Krise von Müttern bzw. Paaren sehr unterschiedlich verteilt ist. Bei LeMasters (1957) sind es $13 \%$ der Paare, die keine Krise erlebten, bei Dyers (1963) und bei Hobbs (1965) sind es $47 \%$ bzw. 82,5\% der Mütter.

Filip (1981) sieht die Geburt des ersten Kindes als kritisches Lebensereignis an und erweitert die Perspektive auf sowohl "nicht gelingende“ und damit krisenhafte, als auch auf "gelingende" Bewältigung dieses Lebensabschnittes. Der Schwerpunkt bei Filip (1981) liegt auf der differenzierten Betrachtung der Art der Verarbeitung eines kritischen Lebensereignisses.

Eine andere Betrachtungsweise bietet Gloger-Tippelt (1985, 1988). Es handelt sich bei ihr um das offene und wertneutrale Konzept der Übergänge bei der Erforschung der Aneinanderreihung der Ereignisse von der Entscheidungsfindung und Planung eines Kindes, Schwangerschaft und Geburt, bis hin zum Beginn der Elternschaft. Die Gesamtheit der Veränderungen, die für Mutter und Vater als Individuum und für das Paar mit dem ersten Kind verbunden in Verbindung gebracht werden, bezeichnet Gloger-Tippelt (1985) als Übergang im Lebenslauf. Die Forscherin kennzeichnet die Übergänge als „zeitlich gedrängte, zum Teil als 
krisenhaft erlebte quantitative und qualitative Veränderungsprozesse“ (1985, S. $54)$.

Aufgrund ihrer Forschungsergebnisse beschreibt Gloger-Tippelt acht idealtypische Verarbeitungsphasen von vor der Geburt bis zum Ende des ersten Lebensjahres. Sie unterteilt vier vorgeburtliche Phasen, eine Geburtsphase und drei Phasen der Umstellung auf die neue Lebenssituation. Die vier vorgeburtlichen Phasen werden in Tabelle 1 dargestellt, wobei der zeitliche Rahmen nur als Orientierungshilfe zu sehen ist.

Tabelle 1: Schwangerschaftsphasen (Gloger-Tippelt, 1988)

\begin{tabular}{|l|l|l|}
\hline 1. Verunsicherungsphase & $\begin{array}{l}\text { bis ca. 12. } \\
\text { Schwangerschaftswoche }\end{array}$ & $\begin{array}{l}\text { Erleben relativ abhängig von } \\
\text { Geplantheit und Erwünschtheit } \\
\text { der Schwangerschaft }\end{array}$ \\
\hline 2. Anpassungsphase & $\begin{array}{l}\text { 12. bis 20. } \\
\text { Schwangerschaftswoche }\end{array}$ & $\begin{array}{l}\text { entgültige Entscheidung für } \\
\text { Schwangerschaft; andere Personen } \\
\text { werden über Schwangerschaft } \\
\text { informiert }\end{array}$ \\
\hline 3. Phase der Konkretisierung & $\begin{array}{l}\text { ca. 20. bis 32. } \\
\text { Schwangerschaftswoche }\end{array}$ & $\begin{array}{l}\text { relativ niedriges Angstniveau, } \\
\text { positive Grundhaltung für weiteren } \\
\text { Verlauf der Schwangerschaft und } \\
\text { Geburt, Sicherheitsgefühl bildet sich } \\
\text { aus }\end{array}$ \\
\hline 4. Phase der Antizipation & $\begin{array}{l}\text { ca. 32. } \\
\text { Schwangerschaftswoche bis } \\
\text { Geburt }\end{array}$ & $\begin{array}{l}\text { Ängste und Labilisierungen werden } \\
\text { wieder stärker; beide Partner leiden } \\
\text { in dieser Phase am stärksten unter } \\
\text { psychosomatischen Beschwerden }\end{array}$ \\
\hline
\end{tabular}

Auch bei Rauchfuss (1986) steht der Prozesscharakter der Schwangerschaft im Vordergrund. Sie sieht die Schwangerschaft als ein Veränderungs- und Adaptionsprozess an. Sie betont, dass der Adaptionsprozess sowohl auf der somatischen wie auch auf der psychosozialen Ebene von Bedeutung ist. 
Ihre Einteilung der Veränderungen im psychosozialen Bereich erfolgt in drei Bereiche:

B Veränderungen auf intrapersoneller Ebene: Schwangerschaft als „point of no return", mit schwerwiegenden Lebensveränderungen, die sowohl Gewinn als auch Verlust mit sich bringen

B Veränderungen auf interpersoneller Ebene: bei der Partnerbeziehung, bei der Beziehung zur Ursprungsfamilie und bei der beruflichen Neuorientierung

B Veränderungen bezüglich des Körperbild: neues Körperbild muss in das bisherige Körperschema integriert werden.

Rauchfuss (1986) sieht in einer nicht gelungenen Anpassung einen möglichen Auslöser für Schwangerschaftskomplikationen.

\subsubsection{Risikoschwangerschaft/Schwangerschaftskomplikationen}

Der Begriff der Risikoschwangerschaft ist breit gefächert. Im Mutterpass des Bundesausschusses der Ärzte und Krankenkassen (1997) werden 52 Schwangerschaftsrisiken genannt, die zu einer Risikoschwangerschaft führen (z.B. Zustand nach Sectio, Adipositas, Mehrlingsschwangerschaft, Diabetes mellitus, usw.). Laut Pschyrembel (1990) ist eine Risikoschwangerschaft vorliegend, wenn „eine Gefährdung des Feten oder der Mutter besteht; Häufigkeit etwas 30\%" (S. 1456).

Im Folgenden werden nur Risiken/Komplikationen aufgeführt, die als Negativkriterium für eine Hausgeburt gelten und/oder tatsächlich im empirischen Teil der vorliegenden Diplomarbeit auftraten. 
Hypertensive Schwangerschaftserkrankungen (HSE; früher auch als EPH-Gestose bezeichnt)

Zu den hypertensiven Schwangerschaftserkrankungen zählen (klinischsymptomatische Einteilung gemäss der Internationalen Gesellschaft für das Studium der Hypertension in der Schwangerschaft, 1986, zit. nach Pschyrembel \& Dudenhausen, 1991) :

1. Gestations-Hypertension

B diastolischer Blutdruck grösser/gleich $90 \mathrm{~mm} \mathrm{Hg}$

B keine Proteinurie

B vorher keine Proteinurie oder Hypertension

2. Gestations-Proteinurie

B Proteinurie grösser $0,5 \mathrm{~g} / \mathrm{im} 24 \mathrm{Std}$.-Urin

B Keine Hypertension

B Vorher keine Hypertension oder Proteinurie

3. Proteinurische Gestations-Hypertension = Präeklampsie

B Kombination von 1 und 2

4. Eklampsie

B Zu den unter 1.-3. genannten Symptomen treten Symptome der drohenden Eklampsie bzw. des eklamptischen Anfalls

Pschyrembel (1990) gibt eine Häufigkeit von ca. 10\% an und als Ätiologie ein multifaktorielles Geschehen mit bekannten prädisponierenden Faktoren (z.B. Mehrlingsschwangerschaft, Erstgebärende, höheres Lebensalter, usw.) an.

Eine seltene, sehr gefährliche Variante der EPH-Gestose ist das

5. HELLP-Syndrom

B Hämolyse

B Pathologisch erhöhte Transaminasen und Bilirubinwerte

B Niedrige Thrombozytenzahlen

(Pschyrembel, 1990). 
Alle Formen der hypertensiven Erkrankungen können zu einer Lebensbedrohung für Mutter und Kind führen (Minderdurchblutung der Plazenta, Blutgerinnungsstörung, Leberkoma). Bringen medikamentöse Behandlungen keine Besserung, muss eine rasche (Schnitt-)Entbindung erfolgen (Pschyrembel \& Dudenahusen, 1991).

\section{Diabetes (gravidarum)}

Der Gestationsdiabetes ist eine Form des latenten Diabetes mellitus:

Bei diesen Frauen sind die Blutzuckerwerte ohne und mit Glukose-Belastungstesten normal, nur unter der Stoffwechselbelastungssituation der Schwangerschaft fällt der Glukose-Belastungstest pathologisch aus. Ganz allgemein gesehen, ist ein Diabetes mit Risiken für Mutter und Kind verbunden, wenn er insulinpflichtig ist, unabhängig vom Auftretenszeitpunkt. Risiken sind z.B. Koma, Missbildungen und intrauteriner Fruchttod (Pschyrembel \& Dudenhausen, 1991; Bund Deutscher Hebammen e.V., 2000).

\section{$\underline{\text { Anämie }}$}

Entsprechend der Mutterschaftsrichtlinien werden drei Formen der Anämie unterschieden:

B Leichte Anämie: $\mathrm{Hb} 10 \mathrm{~g} \%-11 \mathrm{~g} \%$

B Mittelschwere Anämie: $\mathrm{Hb} 8 \mathrm{~g} \%-9,9 \mathrm{~g} \%$

B Schwere Anämie: $\mathrm{Hb}$ unter $8 \mathrm{~g} \%$

Mit Risiken ist zu rechnen (z.B. erhöhte Schockbereitschaft, erhöhtes Infektionsrisiko und Plazentainsuffizienz) wenn klinische Symptome wie Kopfschmerzen und Schwindelanfälle bestehen (Bund Deutscher Hebammen e.V. 2000).

\section{Makrosomie}

Geburtsgewicht über $4500 \mathrm{~g}$ (Pschyrembel \& Dudenhausen, 1991). Damit besteht ein erhöhtes Risiko für die Notwendikgeit eines geburtshilflich-operativen Eingriffs (Geburtsstillstand, Schulterdystokie) und postnatale kindliche Stoffwechselstörungen. 


\section{Wachstumsretardierung}

Eine Mangelgeburt wird durch das Geburtsgewicht in Abhängigkeit vom Schwangerschaftsalter definiert:

Ein Mangelgeborenes ist ein Neugeborenes, dessen Gewicht unterhalb der 10. Perzentile der Standardgewichtskurve liegt. Wachstumsretardierte Kinder sind sehr anfällig für die intrauterine Hypoxie, weshalb die operative Entbindungsrate erhöht ist. Der Verlauf kann sehr akut sein (Pschyrembel \& Dudenhausen, 1991).

\section{Fetale Fehlbildung}

Im weitesten Sinne fasst man unter dem Begriff „Fehlbildung“ jede Störung der pränatalen Entwicklung zusammen, die zu bleibenden morphologischen und/oder funktionellen Veränderungen führt (Pschyrembel \& Dudenhausen, 1991). Dies kann je nach Ausprägung zu einer Lebensbedrohung für das Un- bzw. Neugeborene führen und es bedarf einer Intensivüberwachung.

\section{Mehrlingsschwangerschaft}

„Zwei oder mehr Individuen, die einer synchronen intrauterinen Entwicklung unterliegen (Zwillinge, Drillinge, Vierlinge usw.), wobei sich das intrauterine Wachstum vorzeitig verlangsamt; perinatal besteht für diese Kinder ein überdurchschnittliches Risiko" (Pschyrembel, 1990, S. 1039). Wehenschwäche, vorzeitige Plazentalösung sowie Atonie des Uterus sind häufige und typische Komplikationen.

\section{Thrombose}

Unter einer Thrombose versteht man eine „Blutpfropfbildung; intravital erfolgende intravasale Blutgerinnung“ (Pschyrembel, 1990, S. 1669). Dadurch besteht eine erhöhte Emboliegefahr.

\section{Plazenta praevia}

Die atypische Lokalisation der Plazenta im unteren Uterinsegment nennt man Plazenta praevia. Dabei bedeckt ein mehr oder weniger grosser Teil der 
Plazentafläche die Innenwand des unteren Uterinsegments (Pschyrembel, 1990). Besonders unter Wehen besteht die Gefahr von lebensbedrohlichen Blutungen.

\section{Frühgeburt}

Unter einer Frühgeburt versteht man eine Geburt nach der 28. Schwangerschaftswoche und vor der vollendeten 37. Schwangerschaftswoche (Pschyrembel, 1990). Frühgeburtlichkeit ist eine der Hauptursachen für die neonatale Mortalität und Morbidität (Künzel, 1995). Eine postnatale Intensivüberwachung/-behandlung des Neugeborenen muss gewährleistet sein.

\section{Vorzeitiger Blasensprung}

Hier ist der Blasensprung vor der 37. Schwangerschaftswoche gemeint, da er mit den Risiken einer Infektion und/oder Frühgeburt einhergeht (Pschyrembel \& Dudenhausen, 1991).

\section{Terminüberschreitung}

Unter einer echten Terminüberschreitung versteht Martius (1990), wenn eine rechnerische Schwangerschaftsdauer von mehr als 42 vollendeten Schwangerschaftswochen überschritten wird. Infolge der abnehmenden Plazentafunktion besteht eine erhöhte Gefahr für das Kind. Eine Intensivüberwachung ist notwendig (Pschyrembel \& Dudenhausen, 1991).

\subsubsection{Schwangerschaftskomplikationen und Angst}

In bezug auf Schwangerschaftskomplikationen (z.B. Frühgeburtlichkeit, hypertensive Schwangerschaftserkrankung, usw.) ergab die Durchsicht der Literatur folgende Erkenntnisse, wobei hauptsächlich Komplikationen und Aspekte beleuchtet werden, die im Zusammenhang mit der vorliegenden Diplomarbeit stehen.

Eine Untersuchung von McDonald (1965) ergab, dass Schwangerschaftskomplikationen wie Präeklampsie und vorzeitiger Blasensprung 
(vor der vollendeten 37. Schwangerschaftswoche) mit höheren Hypochondrie- und Angstwerten sowie höheren Werten bei sozialer Introversion einhergehen.

Die Stichprobe beinhaltete nur sehr junge erstgebärende Frauen, die zusätzlich ein extrem niedriges Bildungsniveau aufwiesen (also keine repräsentative Stichprobe), was zu kritisieren ist.

Gorsuch \& Key (1974) konnten zeigen, dass Angst und "life stress" (wie z.B. Krankheiten, Tod des Ehemanns und Wohnortwechsel) unabhängig voneinander zur Entstehung von Schwangerschaftskomplikationen beitragen. Allerdings bestand dieser Zusammenhang nur im dritten und vierten Schwangerschaftsmonat. Im sechsten Schwangerschaftsmonat lag der Angstwert der Frauen mit normalverlaufenden Schwangerschaften sogar signifikant höher als bei den Frauen mit Schwangerschaftskomplikationen. Später gab es keine signifikanten Unterschiede mehr zwischen den normal und abnormal verlaufenden Schwangerschaften. Kritisch anzumerken ist, dass die Stichprobe von Gorsuch \& Key (1974) nicht repräsentativ war, da sie nur aus Frauen mit niedrigem Einkommen und Erstschwangerschaften bestand.

Auch Erickson (1975) fand einen Zusammenhang zwischen Schwangerschaftskomplikationen und psychologischen Variablen (z.B. Depression, Angst, usw.). Neuere Untersuchungen zeigten die gleichen Ergebnisse. So konnten Norbeck \& Tilden (1983) zeigen, dass kritische Lebensereignisse und Stress Einfluss auf den Verlauf der Schwangerschaft haben. Diese Studie wies methodische Mängel bezüglich der Stichprobenzusammensetzung auf, sie bestand aus fast ausschliesslich mehrgebärenden Frauen.

Methodisch bessere Studien gibt es aus den 90er Jahren.

Wimmer-Puchinger (1992) fand in ihrer Studie (grosse Zufallsstichprobe) heraus, dass Frauen mit einer hypertensiven Schwangerschaftserkrankung ein signifikant geringeres Selbstwertgefühl haben und ängstlicher sind. 
Zur Vorhersage eines pathologischen Schwangerschaftsverlaufs erwiesen sich vier Prädiktoren am geeignetsten:

B Allgemeine Ängstlichkeit

B Bewusste Planung der Schwangerschaft

B Geburtsangst

B Mutterbeziehung

Rauchfuss (1996) untersuchte in einer prospektiven Fragebogenuntersuchung den Einfluss der Paarbeziehung auf den Schwangerschaftsverlauf und stellte fest, dass Partnerschaftskonflikte mit einer verkürzten Schwangerschaftsdauer korrelieren.

Zu entgegengesetzten Befunden kamen McDonald \& Parham (1964) und Morcos \& Funke-Ferber (1979). Sie fanden keinen Zusammenhang zwischen Angst und Schwangerschaftskomplikationen. Die von McDonald \& Perham untersuchte Stichprobe war jedoch nicht repräsentativ. Nur sehr junge und unverheiratete Erstgebärende wurden untersucht. Bei Morcos \& Funke-Ferber (1979) fehlen Angaben über Alter, Gravidität und Parität, was für die Interpretation sehr ungünstig ist, da die Repräsentativität dieser Stichprobe in Frage gestellt werden muss.

\subsection{Geburt}

Richter \& Stauber (1990, S. 968) definieren die Geburt nicht nur als "das physiologische Ende der Schwangerschaft, sondern als ein psychosomatisches Ereignis - ein Erlebnis, dass die Frau mit Leib und Seele erfasst. Wohl kaum ein Ereignis im menschlichen Leben ist mit so vielen Geheimnissen umgeben und mit einer solchen Vielfalt an Bedeutungsgehalten versehen worden. So spielt auch der Geburtsvorgang im Denken und Fühlen der Völker eine bedeutsame Rolle, die wiederum je nach weltanschaulicher, rassischer, kultureller und soziologischer Struktur verschieden ist". 


\subsubsection{Physiologie der Geburt}

Die Geburt ist ein Teil des physiologischen Fortpflanzungsvorganges, bei dem es zur Eröffnung des Fruchthalters und der Geburtswege (= Geburtskanal) kommt sowie zur Ausstossung der Frucht, sprich des Kindes (= Geburtsobjekt) und der Nachgeburt. Die treibenden Kräfte sind die Wehen (= Geburtskräfte). Sie haben zur Folge, dass sich der Muttermund öffnet, dass das Kind durch den Geburtskanal tritt und dass sich Plazenta und Eihäute ablösen und ausgestossen werden (Martius, 1990).

Das reife Kind ist ca. 49-52 cm lang und wiegt ca. 3000-3500 g. Der Kopf ist der grösste und härteste Teil des Kindes und geht in 94\% der Geburten voran. Das Verhältnis seiner Grösse zum Becken ist geburtsmechanisch ausschlaggebend für den Geburtsablauf (Pschyrembel \& Dudenhausen, 1991).

\section{Geburtsbeginn}

Die Ursachen des Geburtsbeginns sind noch relativ unbekannt. Was man weiss, ist dass der Wehenbeginn nicht von einem einzelnen Faktor abhängt, sondern vom harmonischen Zusammenspiel einer ganzen Reihe von Faktoren.

Ein sinnvolles Zusammenspiel von

B Mechanische Veränderungen

B Nervale Veränderungen

B Endokrine, chemische Veränderungen

B Intrauterine Reifung des Kindes

bewirkt, dass Schwangerschaftswehen in Geburtswehen übergehen (Martius, 1990;

Pschyrembel \& Dudenhausen, 1991).

Direkt vor dem Geburtsbeginn kommt es zu einem Sensibilitätsanstieg des Myometriums für Prostaglandine und Oxytozin. Der deutliche Anstieg der Oxytozinrezeptoren-Konzentration im Myometrium wird als Ursache der drastischen Zunahme der Oxytozinempfindlichkeit angesehen. Des weiteren soll es zu einer Zunahme der Oxytozinrezeptoren in der Dezidua kommen, die möglicherweise die 
Prostaglandinsynthese stimuliert. Ausserdem ist es gesichert, dass die fetale Hypophyse vor dem Wehenbeginn zunehmend Oxytozin freisetzt, gleichzeitig regt das Oxytozin die Prostaglandinsynthese an. Es wäre denkbar, dass die Reifung des Zusammenspiels zwischen fetalem Hypothalamus und fetaler Hypophyse ein Signal zur Geburtsauslösung ist. So würde der Fetus selbst den Anstoss zu seiner Geburt geben.

Nach Wehenbeginn wird die Wehentätigkeit hauptsächlich über den sogenannten Fergusonreflex (bzw. Wehenreflex) gesteuert. Der Druck des tiefertretenden Geburtsobjekts wirkt auf die Oxytozinsezernierung der Hypophyse anregend, und das Myometrium wird über einen direkten Reflexbogen über das Rückenmark zur Kontraktion angeregt (Pschyrembel \& Dudenhausen, 1991; Martius, 1990).

\section{Geburtsverlauf}

Die folgenden Beschreibungen beruhen auf Pschyrembel \& Dudenhausen (1991), Martius (1990) und Stadelmann (1994).

Der Geburtsverlauf wird in der Regel in vier Phasen eingeteilt:

B Eröffnungsperiode: Wehenbeginn bis ca. 7-8 cm Muttermunsderöffnung

B Übergangsperiode: $7-8 \mathrm{~cm}$ bis zur vollständigen Muttermundseröffnung $(10 \mathrm{~cm})$

B Austreibungsperiode: beginnt mit der vollständigen Muttermundseröffnung und endet mit der Geburt des Kindes

B Nachgeburtsperiode: Plazenta löst sich von ihrer Haftstelle und wird ausgestossen

Die Geburtsdauer wird von den verschiedenen Autoren sehr unterschiedlich angegeben. Durchschnittszeiten für Erstgebärende variieren zwischen 6-24 Stunden und bei Mehrgebärenden zwischen 3-9 Stunden.

Beim regelrechten Geburtsmechanismus verläuft die Geburt des Kindes in Längslage, in Schädellage und als vordere Hinterhauptslage.

Beim Eintritt in den Beckeneingang (= Eintrittsmechanismus) stellt sich der Kopf so ein, dass die Pfeilnaht quer oder leicht schräg verläuft. So passt sich der Kopf dem querovalen Beckeneingang an (Gesetz des kleinsten Zwanges von C.F. Gauss). 
Beim Durchtritt durch die Beckenhöhle (= Durchtrittsmechanismus), tritt der Kopf tiefer und beugt sich, um sich der runden Beckenhöhle besser anzupassen. Nun tritt der Kopf mit führender kleiner Fontanelle, d.h. in stärkster Beugung durch das Weichteilrohr hindurch.

Zum Austritt aus dem Geburtskanal (= Austrittsmechanismus) stemmt sich der Kopf sich mit dem Nacken am unteren Symphysenrand an, um dann aus der tiefen Beugehaltung heraus eine Streckbewegung zu machen. Der Kopf ändert also lediglich seine Haltung, um aus dem Geburtskanal austreten zu können.

Die Schultern treten im Verlauf des Kopfaustrittes im hohen Schulterquerstand in das kleine Becken ein. Die Schulterbreite dreht sich anschliessend im kleinen Becken aus dem hohen Querstand in den tiefen Geradstand, was auch durch die äussere Drehung des Kopfes erkennbar ist. Der Austritt der Schulterbreite erfolgt dann im tiefen Schultergeradstand, also mit gerade stehender Schulterbreite. Abschliessend erfolgt die Geburt des Rumpfes einschliesslich der Hüftbreite zwanglos (bei normal grossen Kindern), da die Hüften mit einem Umfang von ca. $24 \mathrm{~cm}$ eine Formanpassung unnötig machen.

Der Lösungsmechanismus der Plazenta erfolgt durch die Kontraktion und Retraktion des Uterus. Nach Ablauf der ersten oder zweiten kräftigen Nachwehe ist im Normalfall die Plazenta vollständig gelöst. Jedoch sollte der Mutterkuchen sich nach spätestens 30 min gelöst haben. Mit jeder Nachwehe wird die Plazentahaftfläche auf der Uteruswand kleiner und somit wird auch eine sofortige Blutstillung der uteroplazentaren Gefässe sichergestellt. Zusätzlich wird durch die Kreislaufumstellung im uterinen Gefässsystem die Bildung von Gerinnungsthromben eingeleitet. Der normale Blutverlust liegt bei ca. $300 \mathrm{ml}$.

\subsubsection{Pathologie der Geburt/Geburtskomplikationen}

Aufgrund der vielen möglichen Geburtskomplikationen, werden im Folgenden nur die wichtigsten Komplikationen und/oder die im empirischen Teil der vorliegenden Diplomarbeit auch wirklich auftraten, aufgeführt. 


\section{Intrauterine Asphyxie}

Unter der intrauterinen Asphyxie ist eine fetale Hypoxie, einem Erstickungszustand des Fetus mit Sauerstoffmangel und Kohlensäureüberladung zu verstehen.

Die Ursachen sind sehr vielfältig, z.B. mütterliche Anämie, protrahierter Geburtsverlauf, Nabelschnurkomplikationen usw. (Martius, 1990).

Zur Therapie stehen im wesentlichen zwei Möglichkeiten zur Verfügung:

B Intrauterine Reanimation, d.h. Gabe von wehenhemmenden Medikamenten, zur besseren uteroplazentaren Durchblutung

B Operative Geburtsbeendigung

\section{Regelwidrige Geburtsdauer}

Der protrahierte Geburtsverlauf ist wie folgt definiert:

B bei Erstgebärenden über 12 Stunden

B bei Mehrgebärenden über 8 Stunden

Es ist bekannt, dass nach diesen Grenzwerten gehäuft fetale Hypoxien und Azidosen auftreten (Martius, 1990).

Die Ursachen können vielfältig sein, z.B. Wehenschwäche, grosses Kind, Haltungsund Einstellungsanomalien usw.

Die Therapie sollte nicht erst eingeleitet werden, wenn die Geburtsdauer überschritten wird. Je nach Ursache sind die Gabe von Wehenmittel, Schmerz- und Entspannungsmittel usw. indiziert. Ist eine Geburtsbeendigung angezeigt, so erfolgt diese vaginal-operativ (Vacuum- oder Zangenextraktion) oder per Kaiserschnitt.

\section{Pathologie der Wehen}

Generell versteht man unter einer Wehenschwäche Wehen, die entweder zu

B schwach

B kurz

B selten

sind.

Man unterscheidet zwischen einer primären und sekundären Wehenschwäche. Bei der primären Wehenschwäche ist mangelnde Wehentätigkeit von Geburtsbeginn an 
vorhanden. Deshalb kommt die Geburt erst gar nicht recht in Gang. Bei der sekundären Wehenschwäche handelt es sich hauptsächlich um eine Ermüdung der Uterus- und Bauchmuskulatur.

Beide Formen können mit der Gabe von Wehenmitteln behandelt werden, sofern die Schwäche nicht durch ein unüberwindbares Geburtshindernis (z.B. enges Becken usw.) bedingt ist (Pschyrembel \& Dudenhausen, 1991).

\section{Regelwidriger Geburtsmechanismus}

Die Gesichtslage (Haltungsanomalie) ist eine Deflexionslage. Sie führt in der Regel zu einem protrahierten Geburtsverlauf bzw. macht eine Spontangeburt unmöglich. Häufig werden diese Geburten vaginal-operativ bzw. per Kaiserschnitt beendet.

Beim hohen Geradstand (Einstellungsanomalie) bleibt der Kopf über oder im Becken mit der Pfeilnaht im geraden Durchmesser. Normalerweise sollte er sie im queren oder schrägen Durchmesser passieren. Es kommt zu einem Geburtsstillstand in der Eröffnungsperiode und zur Geburtsbeendigung ist eine Schnittentbindung indiziert.

\section{Missverhältnis zwischen Geburtsobjekt und Geburtskanal}

Mass- und Formanomalien des Beckens führen zu protrahierten Geburtsverläufen und je nach Ausprägung der Anomalie auch zum Geburtsstillstand in der Eröffnungs- oder Austreibungsperiode oder machen eine vaginale Geburt unmöglich. Es besteht also ein Kopf-Becken-Missverhältnis.

Um die Geburt zu beendigen (bei protrahierten Geburtsverläufen nicht zwingend notwendig), ist eine vaginal-operative Entbindung oder eine Schnittentbindung nötig.

Abnorme Grösse und Form des Kindes (z.B. Riesenkind) können auch zu einem (Kopf-)Becken-Missverhältnis führen. Die Folgen und die Therapie sind mit denen der Beckenanomalien identisch. 
Regelwidrigkeiten der Plazenta und in der Nachgeburtsperiode

Vorzeitige Plazentalösung

Pschyrembel \& Dudenhausen (1991) definieren die vorzeitige Plazentalösung als teilweise oder vollständige Ablösung der normal sitzenden Plazenta von ihrer Haftfläche vor der Geburt des Kindes. Es kommt zu Blutungen aus den mütterlichen und oft auch aus den kindlichen Gefässen im Bereich der Haftfläche. Es besteht Lebensgefahr für Mutter und Kind. Die Ursache kann in den meisten Fällen nicht geklärt werden.

Behandelt wird die vorzeitige Plazentalösung mit der sofortigen Geburtsbeendigung. Je nach Geburtsfortschritt per vaginal-operativer Entbindung oder Schnittentbindung.

\section{Lösungsstörungen der Plazenta}

Bei einer Plazenta accreta, increta und percreta handelt es sich um eine anatomische Lösungsstörung, bei der die Zotten über die Dezidua basalis hinaus in die Gebärmutterwand vorgewachsen sind.

Zur Behandlung dieser Störung wird eine manuelle Plazentalösung in Narkose vorgenommen (Martius, 1990).

\section{Verstärkte Blutungen in der Nachgeburtsperiode}

Unter einer atonischen Nachblutung ist eine verstärkte Blutung aus der Gebärmutter zu verstehen, die durch die fehlende Kontraktion des Uterus verursacht wird.

Therapeutisch muss eine intravenöse Wehenmittelgabe erfolgen, wenn die Blutung danach nicht sistiert, muss die Gebärmutter operativ entfernt werden (abdominelle Hysterektomie).

Eine andere Variante der verstärkten Nachblutung ist die Blutung aus der Plazentahaftstelle bei unvollständig gelöster Plazenta.

Hier muss eine Curettage erfolgen. 
Geburtsverletzungen der Mutter

Uterusruptur

Eine Gebärmutterzerreissung kann infolge Wandschädigung (z.B. narbiger Uterus durch vorausgegangene Operation) oder Überdehnung (z.B. Querlage, Missverhältnis zwischen Kopf und Becken) entstehen.

Um die Lebensgefahr von Mutter und Kind abzuwenden muss die Geburt sofort operativ beendet werden und eine abdominelle Hysterektomie vorgenommen werden (Martius, 1990; Pschyrembel \& Dudenhausen, 1991).

\subsubsection{Angst und Geburt}

\subsubsection{Geburtsvorbereitung}

Die Geschichte der Geburtsvorbereitung hat ihre Anfänge in den 30er Jahren. Unterschieden werden muss aber zwischen einer familiären und institutionellen Geburtsvorbereitung. Erstere gab es schon seit je her. Doch durch die zunehmende Urbanisierung, die Entwicklung von der Grossfamilie zur Kleinfamilie und dem Trend weg von der Hausgeburt zur Klinikgeburt verloren die Frauen diese Lernmöglichkeiten. Die ersten institutionellen Angebote waren sogenannte Mütterkurse, beeinflusst durch den Geburtshelfer Dick-Read (Hauffe, 1987). Bekannt wurde er durch sein „Angst-Verspannungs-Schmerz-Syndrom“, einem Circulus vitiosus:

Angst führt zu Verkrampfungen, Verkrampfungen zu Schmerz, der wiederum zu Angst führt, was insgesamt dem natürlichen Geburtsverlauf entgegenwirkt (DickRead, 1972).

Dies entspricht dem allgemeinen Prinzip, wie es in der Angstforschung ebenso wie in der Psychophysiologie (Pinel, 2002; Barlow, 1988; Larbig \& Birbaumer, 1986) beschrieben wird. 
Seine geburtsvorbereitender Unterricht basierte auf vier Schwerpunkten:

B Wissen über die Vorgänge bei Schwangerschaft und Geburt

B Erlernen von richtigem Atmen (tiefe Bauchatmung)

B Entspannung während Schwangerschaft und Geburt

B Leichte Turnübungen zur Erhöhung der Leistungsfähigkeit (für die Geburtsarbeit notwendig)

In den 70er Jahren wurde in Deutschland die Lamaze-Methode bekannt (in Europa in den 50er Jahren). Seine Methode wird als Erziehung zur Geburt bezeichnet. Sie gründet darauf, dass Schwangere lernen, geburtsbezogene negative und schmerzauslösende Reflexe abzubauen und sie durch positive auszutauschen. Das heisst, dass die zu erwartenden Schmerzen schon in der Schwangerschaft eliminiert werden können. Die Frauen lernten die Geburt als ein positives Ereignis zu verstehen und den Wehen mit gezieltem Atem- und Entspannungsverhalten zu begegnen. So wurde den Frauen ermöglicht, eine Geburt mit geringeren Schmerzen zu erleben. Bei dieser Methode wird im Gegensatz zu Dick-Read, auch der Partner in die Geburtsvorbereitung involviert (Hauffe, 1987).

Die psychosexuelle Geburtsvorbereitung von Sheila Kitzinger wurde in der zweiten Hälfte der 70er Jahre in Deutschland publik.

,... es ist eine Vorbereitung nicht nur für die schwangere Frau, sondern auch für den Mann, und sie ist nicht nur auf den Akt der Geburt bezogen, sondern auf Menschen in einer Beziehung, die von Liebe und wechselseitiger Abhängigkeit getragen ist. Die subjektive Erfahrung des Gebärens ist in Wirklichkeit nicht nur an die Gegenwart, sondern auch an die Vergangenheit gebunden; sie ist die Auswirkung der Erziehung und der Kindheitserfahrungen der Frau, sie ist nicht einfach eine Sache des intellektuellen Wissens und der Vorstellungen von Gebären, die mehr oder weniger zutreffend sind, sondern auch des inneren Körperbildes, das in der Kindheit angelegt wurde und sich später im Berühren und Berührtwerden, im Beieinanderliegen und in der Beziehung der Körper zueinander ausgestaltet" (Kitzinger, 1980, zitiert nach Hauffe, 1987, S. 316). 
Laut Kitzinger beteiligt sich die Frau bewusst bei der Geburt, so dass Selbstkontrolle, Selbstbestimmung und Entscheidungen auf freiwilliger Basis zu einer aktiven Zusammenarbeit mit den Geburtshelfern führen. Sie ist nicht mehr passives leidendes Werkzeug. Die Neuorientierung der Geburtsvorbereitung in Deutschland ist grösstenteils Sheila Kitzinger zu verdanken.

Hauffe (1987) beschreibt wie die heutige Geburtsvorbereitung aussehen sollte: „Geburtsvorbereitung ist heute der Ort, sich Wissen über Geburt zu verschaffen. Mit Wissen ist hier nicht allein geistiges Wissen gemeint, sondern auch körperliches und emotionales" (S. 317).

In psychologischer Fachsprache ausgedrückt, ist Geburt ein Problemlöse- und Entscheidungsprozess. Die Persönlichkeit der Gebärenden, ihr im Verlauf ihres Lebens entwickelter Charakter, im täglichen Leben Entscheidungen zu fällen, lebt auch während der Geburt. Die schwangeren Frauen sind in der Lage ihre Körperund Gefühlssignale zu erkennen und zu deuten, um sie in für sich und das Kind positives Verhalten umzusetzen, was im Geburtsvorbereitungskurs massgeblich unterstützt werden kann.

Hauffe (1987) postulierte drei Schwerpunkte in der Geburtsvorbereitung:

B Körperarbeit (Übungen zum Bewusstwerden körperlicher Vorgänge, Entspannung, Atmung, Massage usw.)

B Informationsarbeit (Physiologie von Schwangerschaft, Geburt und Wochenbett, Ernährung usw.)

B Gespräche innerhalb der Gruppe (für das Verständnis zwischen den werdenden Eltern)

Körperarbeitsmethoden waren bisher strikt vorgegeben, vor allen im Bereich der Atmung. Vorgegebene Techniken brachten keine langfristigen Erfolge. Oft hatten die Frauen das Gefühl versagt $z u$ haben oder empfanden den Geburtsvorbereitungskurs als nutzlos. Diese Gefahr wurde auch Neumann (1987) genant, wenn es in der Geburtsvorbereitung nur um Schmerzlinderung geht (Grundidee der geburtsvorbereitenden Arbeit). 
Atemarbeit in der modernen Geburtsvorbereitung unterliegt keiner Technik. Jede Teilnehmerin, jeder Teilnehmer lernt seinen persönlichen Atemfluss kennen, nicht ein Zählmuster, sondern Erlangen ein körperliches Wissen über ihre Atemvorgänge, Erfahrung der energetische Kraft des eigenen Atems.

Für den Themenkreis Entspannung gilt Ähnliches. Der Schwerpunkt sollte auf dem Weg zur Entspannung gelegt werden und nicht nur auf das Ziel, die Entspannung. Die Schwangerschaftsgymnastik hat nicht mehr die Intension, körperliche Fitness zu trainieren (würde auch nicht mehr in den zeitlichen Rahmen passen), sondern hat die Funktion, dass körperliche Vorgänge und Zusammenhänge kennengelernt werden (Neumann, 1987).

Diese so gewonnene Sicherheit in die eigenen körperlichen, geistigen und seelischen Fähigkeiten macht ein befriedigendes Erleben von Schwangerschaft, Geburt und Elternschaft möglich. Unter „befriedigendem Erleben“ versteht Hauffe (1987) einen subjektiven Begriff. "Wir dürfen nicht auch hier wieder nur normativ denken (Beispiel: Hauptsache, eine Geburt ist kurz.). Befriedigend kann auch eine Geburt sein, die nicht den persönlichen Vorstellungen und Werten einer Hebamme oder Arztes entspricht. Auch hier neigen wir dazu, für andere mitzuentscheiden“ (S. 319).

Hauptsächlich scheint der Vorteil geburtsvorbereitender Massnahmen der zu sein, dass Frauen besser lernen, sich aus der passiven Rolle der Gebärenden zu lösen, sich Unsicherheiten und Konflikte reduzieren, die mit der Schwangerschaft in Verbindung stehen. So wird die Fähigkeit, bei der Geburt selbst aktiver eingebunden zu werden, eigene Erwartungen und Wünsche zu äussern und die Geburt aktiv zu bewältigen, in geburtsvorbereitenden Kursen unterstützt (DaviesOsterkamp und Beckmann 1982).

Dass eine ganzheitliche Geburtsvorbereitung einen positiven Einfluss auf Schwangerschaft, Geburt und Elternschaft hat, scheint auch objektivierbar zu sein: 1979 wurde am Aachener Luisenhospital festgestellt, dass die Anzahl operativer Entbindungszahlen bei ganzheitlich vorbereiteten Paaren auffallend niedrig war 
(7,5\%), im Gegensatz zu anderen (13,8\%), die dort geboren haben (Hauffe, 1987). Stähler \& Stähler (1982) konnten in einer Perinatalstudie von 1963 bis 1971 beweisen, dass bei Frauen, die „psychoprophylaktisch“ vorbereitet waren, die perinatale Mortalität, die Auftretensrate von EPH-Gestosen und die Frühgeburtsrate signifikant tiefer lagen.

\subsubsection{Psychische Korrelate des Geburtsverlaufs}

Nicht nur viele biologische Faktoren beeinflussen den Geburtsverlauf, sondern auch psychische Faktoren (Hüter, 1966). Diese Faktoren dienen vor allem dann zur Erklärung von Regelwidrigkeiten, wenn die physiologisch-anatomischen Konstellationen den pathologischen Geburtsverlauf nicht erklären können (Lukesch, 1982).

Über den Einfluss psychologischer Faktoren auf die Geburt wird schon sehr lange berichtet. Bereits im Alten und Neuen Testament wurde der Einfluss solcher Faktoren erwähnt, wie auch von frühen griechischen Ärzten und europäischen Gelehrten des Mittelalters (Istvan, 1986).

Im folgenden Abschnitt wird ein Überblick der Literatur zu diesem Thema gegeben. Schwerpunktmässig geht es um Angst, welches für die vorliegende Diplomarbeit relevant ist und in der Literatur auch am häufigsten diskutiert wird.

Bis in die 60er Jahre sind die Ergebnisse der damals aktuellen Forschungsarbeiten mit Vorsicht zu geniessen, da sie schwere methodische Mängel aufweisen. Die Interpretationen dieser retrospektiven Untersuchungen sind sehr fragwürdig. So z.B. die Studie von Kapp, Hornstein \& Graham (1963). Es wurde ein Zusammenhang von unkoordinierter Wehentätigkeit und Angst festgestellt, wobei es sich hier wie gesagt um ein retrospektives Untersuchungsdesign handelt.

Davids \& DeVault (1961) sowie Davids, DeVault \& Talmadge (1962) waren unter den ersten Forscher, die mit methodisch besseren prospektiven Designs arbeiteten. Die Ergebnisse ihrer Untersuchungen sprachen dafür, dass Angst ein Prädiktor für geburtshilfliche Probleme ist. Ein weiterer Vorteil dieser Studien war die Angsterfassung durch mehrere Messinstrumente. Methodische Mängel weisen 
diese Studien aber trotzdem auf, da für die Interpretation wichtige Angaben, wie der Parität fehlen und die Stichprobe nur mehrgebärende Frauen enthält.

Auf diesem Gebiet wurde in den 60er Jahren am meisten von McDonald und diversen Co-Autoren geforscht. Es konnte eine Verbindung zwischen Angst und Geburtskomplikationen gezeigt werden (McDonald \& Christakos, 1963; McDonald, Gynther \& Christakos, 1963). McDonald (1968) stellte wiederum fest, dass eher ängstliche und auch depressive Frauen komplikationsreichere Geburtsverläufe haben. Zu kritisieren ist bei oben genannten Studien, dass jeweils Angaben über die Parität fehlen, weshalb die Interpretation eingeschränkt ist.

Erhöhte Angstwerte im ersten Trimenon der Schwangerschaft und Geburtskomplikationen wurden von Gorsuch \& Key (1974) (methodische Mängel dieser Studie siehe oben) in Zusammenhang gebracht. Die Studienergebnisse von Rizzardo et al. (1988), welche als aussagekräftig anzusehen sind (repräsentative Stichprobe), unterstützten diesen Zusammenhang ebenfalls. Diese Verbindung konnte auch für den letzten Trimenon von Crandon (1979) festgestellt werden. Die Interpretation letzterer Studie ist durch das Fehlen von Angaben über Gravidität und Parität begrenzt.

Eine Verbindung zwischen protrahierten Geburtsverläufen und hoher Ängstlichkeit konnte in vielen Studien gezeigt werden (z.B. Kartchner, 1950, zit. nach Lukesch, 1981; Falorni, Fornasarig \& Stefanile, 1979; Beck, Siegel, Davidson, Kormeier, Breitenstein \& Hall, 1980). Anzumerken ist auch hier, dass die Interpretationen mit Vorsicht zu betrachten sind, da alle Studien methodische Mängel, wie z.B. retrospektives Design, kleine Stichprobengrösse und Ausserachtlassen von soziodemographischen Daten, aufweisen.

Lukesch \& Lukesch (1976) konnten dies auch für die Beziehung Geburtsangst und Geburtsdauer bestätigen. Nach der Theorie des Angst-Spannungs-SchmerzKreislaufs von Dick-Read (1972) haben Ängste einen wesentlichen Einfluss auf die verlängerte Geburtszeit (siehe oben). 
Eine allgemein erhöhte Ängstlichkeit während der Schwangerschaft wurde häufig durch eine psychosoziale ungünstige Lebenssituation (z.B. Arbeitslosigkeit, alleinerziehend, finanzielle Probleme) der Schwangeren oder durch bewusste oder unbewusste Mutterschaftskonflikte erklärt (Davies-Osterkamp \& Beckmann, 1982). Hauptsächlich bei unsicherer Zukunft, bei einer erschwerenden psychosozialen Situation, beim Vorhandensein einschneidender Lebensveränderungen und bei der Ablehnung des Kindes traten ebenso gehäuft (Schwangerschafts- und) Geburtskomplikationen auf (z.B. Gorsuch \& Key, 1974; Zuckerman et al. 1963, Hetzel et al. 1961, zit. nach Lukesch, 1981).

Andere Autoren brachten komplikationsreiche Geburten mit erhöhten Neurotizismuswerten, Depressionsneigung (emotionale Labilität), erhöhte vegetative Labilität, und niedrigeres Selbstwertgefühl in Zusammenhang (Pajntar, 1972, zit. nach Lukesch, 1982; McDonald \& Christakos, 1963).

Die empirischen Evidenzen lassen sich wie folgt zusammenfassen. Auffälligkeiten im Gebärverhalten sind vor allem verbunden mit (Lukesch, 1981, 1982):

B Allgemeinem Angstniveau

B Aspekten der psychischen Instabilität

B Informationsgrad über Schwangerschaft/Geburt (bei Erstgebärenden)

B Erfahrungen bei vorausgegangenen Geburten

B Beziehung zur Sexualität, Sexualbiographie, einschliesslich Menstruationsvorgeschichte

Neuere und methodisch bessere Untersuchungen sind sehr rar:

Ein erhöhtes Risiko für einen Notfallkaiserschnitt wurde bei Frauen mit erhöhter Geburtsangst und allgemeiner Ängstlichkeit von Ryding, Wijma, Wijma \& Rydhstrom (1998) bei einer sehr grossen Stichprobe $(n=1981)$ gefunden. In dieser Studie wurden mögliche konfundierende Variablen (wie z.B. Alter, Parität, usw.) kontrolliert und es wurde sowohl Geburtsangst als auch allgemeine Angst erfasst. Für beide Variablen konnte der Zusammenhang bestätigt werden. 
Auf der anderen Seite gibt es Autoren, die oben genannten Zusammenhänge zwischen Angst und Geburtskomplikationen bzw. -dauer nicht bestätigen konnten. So McDonald \& Parham (1964), die keine signifikanten Unterschiede bezüglich Angst zwischen den Gruppen mit und ohne Komplikationen bei ihrer Untersuchung fanden. Es bestand auch kein Zusammenhang zwischen Angstwerten und Geburtsdauer (kritische Anmerkung zur Studie siehe oben). In der Studie von Edwards \& Jones (1970) zeigte sich kein Zusammenhang zwischen Angst und Komplikationen. Allerdings wurden nur unverheiratete Frauen mit Erstschwangerschaften (ohne Altersangaben) untersucht, was die Aussagekraft dieser Studie schwächt.

Neuere und methodisch bessere Untersuchungen wie von Beck et al. (1980) und Molfese, Bricker, Manion, Beadnell, Yaple \& Moires (1987) konnten die Verbindung zwischen Angst und Komplikationen ebenfalls nicht bestätigen. In diesen Studien wurde der Einfluss möglicher konfundierenden Variablen kontrolliert, und es wurden repräsentative Stichproben untersucht. Als aussagekräftig ist auch die Untersuchung von Perkin, Bland, Peacock \& Anderson (1993) anzusehen, da sie keine gravierenden methodischen Mängel (z.B. Kontrolle von Drittvariablen, grosse repräsentative Stichprobe) aufweist. Diese Studie konnte kein Zusammenhang zwischen Angst und der Auftretenswahrscheinlichkeit von Komplikationen beweisen.

\subsection{Angst und Entbindungsform}

Eine Geburt kann zugleich als ein psychobiologischer Vorgang und als eine psychosoziale Situation betrachtet werden. Die Wirkung der Situation durch psychosomatische Formen der Anpassung, Angstbewältigung oder auch Symptombildung auf den biologischen Vorgang sind meist noch ungeklärte Zusammenhänge.

Bei der Mehrzahl der Geburten kann man annehmen, dass die biologischen Vorgänge auch die psychosozialen Situationen mitbeeinflussen, da Geburten 
Situationen der entspannten Ruhe und die Hilfe anderer verlangen. „Formen der Geburtshilfe sind in allen Kulturen bekannt, so dass die natürliche Geburt ein abstraktes Konzept bleibt; jede Kultur stellt für die Gebärende Situationen bereit, die den biologischen Vorgang modifizieren und interpretieren" (Mead \& Newton 1965, zit. nach Davies-Osterkamp und Beckmann 1982, S. 493).

In unserem Kulturkreis gibt es hauptsächlich drei typische Entbindungsformen:

B Klinikgeburt

B Ambulante Klinikgeburt

B Hausgeburt (bzw. andere Formen der ausserklinischen Geburt)

Eine psychosoziale Situation wird hauptsächlich durch die formellen und informellen Rollen der Beteiligten erklärt. Die Geburt in der Klinik bedeutet für die Gebärende, dass sie mit vielen verschiedenen Personen bzw. Berufsgruppen in Kontakt tritt: Hebammen, Geburtshelfer, Narkosearzt, Säuglingsschwestern, Stationsschwestern und die hierarchische Verteilung dieser und anderer wie Chefarzt, Oberarzt, Oberschwester usw. Das Personal in grossen Kliniken ist oft stark belastet, so dass ihre Rollen hauptsächlich betriebsorientiert und nicht patientenorientiert sind. Die Gebärende muss sich im Extremfall der Terminierung des Geburtstermins, der Einleitung und automatischen Aufzeichnung der Wehen und Herztöne, der Vorbereitung und Legen eines Periduralkatheters usw. unterwerfen. Im Bereich der informellem Rollen ist die Gebärende in einer ängstlich abhängigen Stellung gegenüber einer omnipotent fantasierten Klinik. Dies kann auf der einen Seite als Verlust an Selbstbestimmung und emotionaler Geborgenheit erlebt werden oder andererseits als Gewinn an Sicherheit betrachtet werden.

Ängstlich abhängige Frauen mit zu erwartenden Geburtskomplikationen empfanden die Überwachung durch Geräte zu meist als Sicherheit und Geborgenheit. Doch wurden diese Geräte auch als "mechanische Monster" angesehen und haben die Geburtsängste eher verstärkt als abgeschwächt (DaviesOsterkamp und Beckmann, 1982). 
Die bei uns bis in die 50er Jahre übliche Hausgeburt entspricht dem anderen Extrem einer psychosozialen Situation. Eine Hausgeburt verläuft im Kreise vertrauter Menschen, einer Hebamme und eventuell eines(r) Arztes/Ärztin und der Familie (auch Freunde), welche so sie die psychosoziale Situation definieren. Die Eltern können sofort mit dem Neugeborenen in Kontakt treten. Die Situation wird mehr von der Gebärenden selbst bestimmt und sie kann sich besser auf die Bedürfnisse ihres Kindes einstellen. In der häuslichen Umgebung kann der Mutter mehr emotionale Zuwendung und Hilfe angeboten werden (Davies-Osterkamp und Beckmann, 1982).

In Deutschland finden Geburten zu ca. 99\% in Krankenhäusern statt (Gesellschaft für Qualität in der ausserklinischen Geburtshilfe e.V., 2001). Entbindungen werden immer mehr wie gefährliche akute Krankheiten betrachtet und dem entsprechend behandelt. Macfarlane (1978) ist der Meinung, dass somit fast alle Geburten „stillschweigend zu Risikogeburten deklariert werden“. Die Mutter wird stillschweigend erpresst, indem man ihr vorwirft, durch eine Hausgeburt ihre eigene Gesundheit und die des Neugeborenen zu gefährden (zit. nach DaviesOsterkamp und Beckmann, 1982, S. 495).

Alle im Folgenden erwähnten Forschungsarbeiten konnten miteinander verglichen werden, da es sich um ähnlich kontrollierte Studien handelt. Es wurden immer nur geplante, risikoarme Geburten miteinander verglichen. Folgende Studien sind methodisch durchweg gut (grosse Stichproben, Kontrolle von Störvariablen usw.).

In Holland beträgt die Rate an Hausgeburten ca. 35\%. Bei der Durchsicht verschiedener Forschungsartikel konnte kein Zusammenhang zwischen einer höheren perinatalen Morbidität und Hausgeburt in Holland gefunden werden (Treffers \& Laan, 1986; Treffers, 1990). Ganz im Gegenteil, die Hausgeburt scheint sogar sicherer als die Klinikgeburt zu sein, auf keinen Fall gefährlicher (Kraker zit. nach Zimmermann, 1998; Wiegers, Keirse, van der Zee \& Berghs, 1996). 
Einheitlich sind die Ergebnisse empirischer Studien, die die Sicherheit von Hausgeburten untersuchten. Es wurde gezeigt, dass Hausgeburten genauso sicher sind wie Klinikgeburten.

Viele neuere internationale Studien belegen dies. Methodisch gute Untersuchungen aus der Schweiz, Neuseeland, Kanada und England kamen zu dem Ergebnis, dass eine geplante Hausgeburt nicht mit einem höheren Risiken für Mutter und Kind verbunden ist (Ackermann-Liebrich, Voegeli, Gunter-Witt, Kunz, Zullig, Schindler \& Maurer, 1997; Gulbransen, Hilton, McKay \& Cox, 1997; Janssen, Lee, Ryan, Etches, Farquharson, Peacock \& Klein, 2002; Macfarlane, McCandlish \& Campell, 2000). Bei den genannten Studien wurde darauf geachtet, dass die Untersuchungsgruppen vergleichbar waren. Es wurden grosse Stichproben untersucht, die bezüglich soziodemographischer und schwangerschaftsspezifischer Merkmale keine Unterschiede aufwiesen („Matching-Prinzip“).

Laut einer offiziellen Verlautbarung zum Thema Geburtshilfe der Weltgesundheitsorganisation (WHO, 1987, zit. nach Zimmermann, 1988) gibt es keinen wissenschaftlichen Anhaltspunkt, der belegt, dass eine geplante Hausgeburt für eine Frau, die einen normalen Schwangerschaftsverlauf hat, mehr Gefahren mit sich bringt als eine Geburt in der Klinik.

Eine Hausgeburt scheint gegenüber einer Klinikgeburt gewisse Vorteile aufzuweisen, um nur einige aufzuzählen (Kelm-Kahl, 1990):

B Keine massive Beeinflussung der Frau, durch z.B. Wehenmittelgabe, Gabe von Schmerzmitteln, welche Morphin-Abkömmlinge sind (wirken atemdepressiv, negativer Einfluss auf Mutter-Kind-Bindung etc.)

B Gewohnte Umgebung, vertraute Menschen: Hebamme ist bekannt und erwünscht (in der Regel kein Schichtwechsel), keine Gerätschaften , die an einen Operationssaal erinnern. So wird Stress minimiert, es wird weniger Adrenalin freigesetzt, was u.a. wehenhemmend wirken kann

B Häusliche Umgebung bietet geringeres Infektionsrisiko, zumal nur eine Person vaginal untersucht (in der Klinik häufig zwei und mehr Personen) 
B 1:1 Betreuung, in Kliniken oft Personalmangel, laut Ärzte Zeitung (31.8.1989) besteht zu ungünstigen Zeiten ein um 50\% höheres Geburtsrisiko

B Keine Dauerüberwachung mit der Cardiotokographie (CTG), sondern intervallmässige Kontrolle mit Stethoskop. Laut WHO gibt es keine Vorteile bei einer Dauerüberwachung, wenn Mütter und Kinder gesund sind. Nur stieg die Kaiserschnittrate um mindestens das Doppelte bei CTG-Überwachung, und der Kaiserschnitt bringt für die Mutter eine viel höhere Mortalitätsrate, ca. zehnfach so hoch als bei allen anderen Geburten.

In einer Stellungnahme der Deutschen Gesellschaft für Gynäkologie und Geburtshilfe zur Hausgeburtshilfe (veröffentlicht in Linder, 1994, S. 228) lehnt jene „die Hausgeburtshilfe, wie auch die Praxisgeburtshilfe wegen ihrer typischen und immanenten Gefahren für das Kind ab.“

Gestützt wird diese Aussage durch diverse Forschungsarbeiten, die zeigten, dass die perinatale Mortalität in Holland grösser als in Deutschland ist und die perinatale Mortalität in Holland in den Regionen am höchsten ist, in denen auch der Anteil an Hausgeburten am grössten ist (Doornbos et al., 1987; Hoogendoorn, 1978; zit. nach Hickl \& Berg, aus Briefwechsel in Linder, 1994).

Kritisch anzumerken ist, dass der Vergleich der perinatalen Mortalität äusserst gewagt ist, da unterschiedliche Definitionen in europäischen Ländern bestehen und auch ungeplante Hausgeburten mit in die Analyse einflossen. Des weiteren wurden von der Gesellschaft keine anderen Autoren genannt, die widerlegen, dass Regionen mit einer hohen Rate an Hausgeburten eine höhere perinatale Mortalität aufweisen, so z.B. Treffers \& Laan (1986) und Treffers (1990).

Studien, die sich mit Angst in Abhängigkeit der Entbindungsform beschäftigen, sind sehr spärlich. Windsor-Oettel (1994) untersuchte diesen Sachverhalt an einer grossen Population in einer deutsch-holländischen Vergleichsstudie. Es konnte kein signifikanter Unterschied bezüglich Angst in Abhängigkeit der Entbindungsform festgestellt werden. Jedoch war dieser Unterschied nur sehr knapp nicht signifikant $(p=0,06)$, was zeigte, dass zumindest eine Tendenz dahingehend besteht. 


\subsection{Erklärungsmechanismen für die Wirkung von Angst auf den Schwangerschafts- und Geburtsverlauf}

Molinski (1975) lieferte einen Erklärungsansatz für die Wirkung von Angst auf der Verhaltensebene beim Schwangerschafts- und Geburtsverlauf. Aufgrund seiner Untersuchungen kam er zum dem Schluss, dass der psychische Zustand, wie Angst der schwangeren bzw. gebärenden Frau direkt in Verhaltensabläufe umgesetzt wird, die im ungünstigen Fall in einen gestörten Geburtsverlauf münden können. So brachte Molinski (1975) z.B. angsterfülltes Gebärverhalten und Muskulaturverkrampfung, Schreien, Strampeln oder retentives Gebärverhalten und Trotz, Widerspenstigkeit, Beckenbodenanspannung in Zusammenhang. Ausser Angst kamen auch andere Affekte bei der Geburt zum Tragen. Vor allem auch Ärger führte zu einem gestörten Gebärverhalten (Molinski, 1976, 1970, 1968 zit. nach Lukesch, 1981). Als Erklärungsmodell wurde bei Molinski (1976, 1970, 1968 zit. nach Lukesch, 1981) eine direkte Umwandlung des psychischen Zustands der Frau in „offen zutage tretende Verhaltensweisen angenommen, die in bezug auf den Geburtsverlauf als dysfunktional zu werten sind“ (Lukesch, 1981, S. 76).

Weitere Erklärungsmuster konnten aus dem Angst-Spannungs-Schmerz-Kreislauf (nach Dick-Read, siehe oben) abgeleitet werden. Wehen führen zur Auslösung von Angst. Die Angstreaktionen siedeln sich auf drei Ebenen an, bestehend aus „affektiven, vegetativen (physiologischen) und muskulären Spannungszuständen“ (Lukesch, 1981, S. 76) mit jeweils typischen Wirkungen. Diese führen zur Verengung der Gefässe sowie zu Muskelverkrampfung, gefolgt von einer erschwerten Blutversorgung und einem Sauerstoffmangel (Hypoxie) in den Geweben, die vor allem unter der Geburt besonders belastet werden. Die Sauerstoffmangelversorgung der Gebärmutter bewirkt selbst schon gesteigerte Schmerzempfindungen. Zudem ist durch die Muskelverkrampfung die Erweiterung des Muttermundes erschwert. Alles in allem wird dadurch die Schmerzintensität aufgrund reaktiver und produktiver Abläufe bei der gebärenden Frau höher. Folglich kommt es zu einem verzögerten Geburtsverlauf und zu vermehrten Schmerzen. Dies wiederum ist die Ursache für eine erhöhte Angstempfindung. Der so entstandene Angst-Spannungs-Schmerz-Kreislauf stellt folglich einen Circulus 
vitiosus dar und hat eine aufschaukelnde Wirkung der negativen Empfindungen zufolge (Lukesch, 1981). Dieser Zusammenhang wurde empirisch nachgewiesen (z.B. Lukesch \& Lukesch, 1976). Siehe dazu Abbildung 1.

Abbildung 1: Angst-Spannungs-Schmerrz-Syndrom (Lukas, 1959; aus Lukesch, 1981)

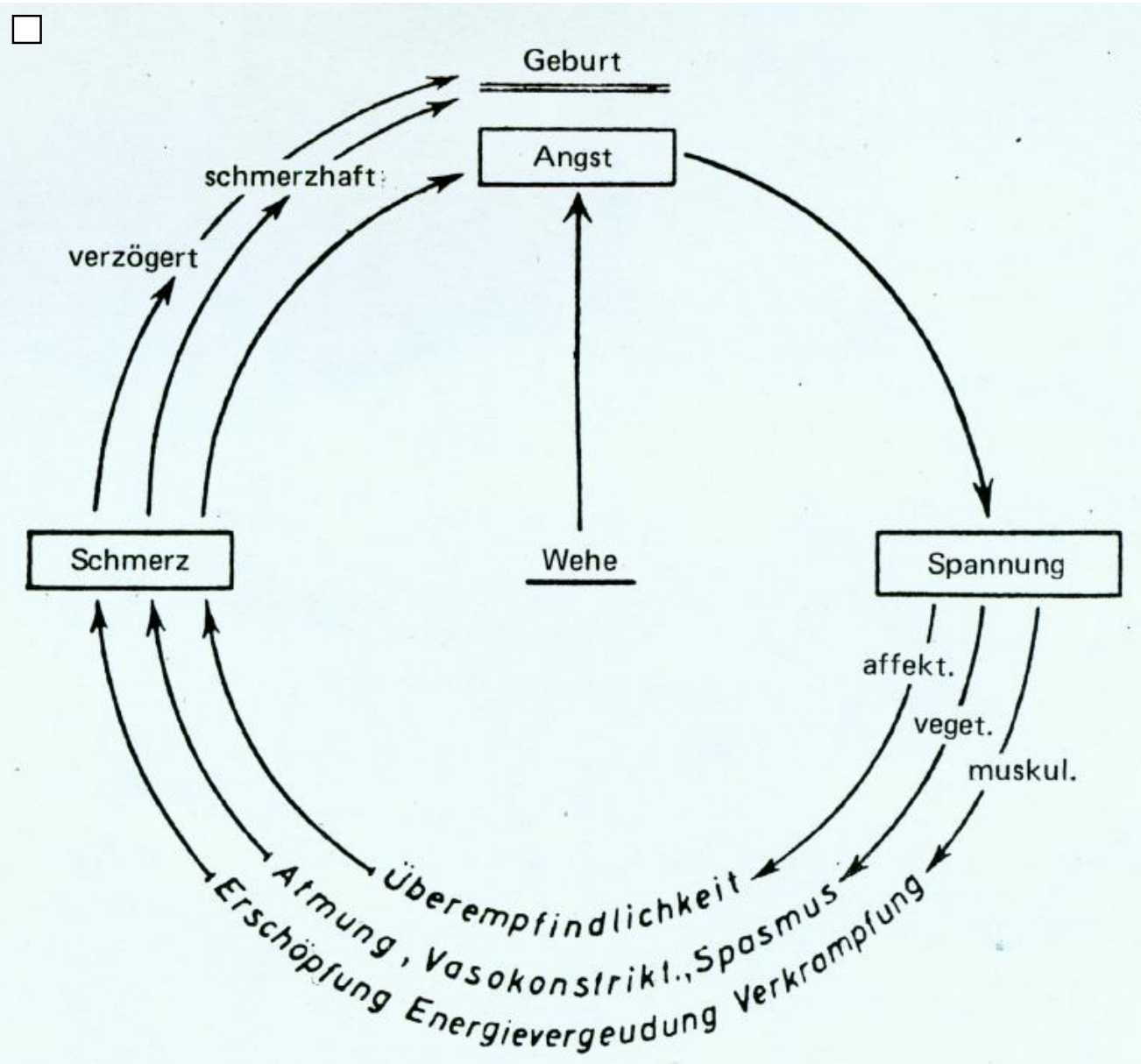

Bayer und Hoff (1959) konnten aufgrund ihrer Untersuchungen zeigen, dass durch Angst und Schmerz stärkemässig vorherrschende Isthmuskontraktionen ausgelöst werden, in Folge dessen es direkt zu einer Verzögerung des Geburtsablaufs und ausserdem zur Hemmung der Wehen kommt.

Kelly (1962) untersuchte den Einfluss von Angst auf die Gebärmutter auf hormoneller Ebene. Er fand heraus, dass Stress-Situationen wie Angst zu einer Veränderung der Gebärmuttermotilität führen, die auf Veränderungen im Adrenalinspiegel zurückzuführen sind. 
Wenn es infolge von Angst zu einem Blutdruckanstieg kommt (Pinel, 2002), der nicht nur kurzfristig ist und den Wert von $135 / 85$ mmHg übersteigt, kann dies zu einer Minderdurchblutung der feto-plazentaren Einheit führen, was eine Minderversorgung des Kindes zu Folge hat (Pschyrembel \& Dudenhausen,1991).

Die oben genannten Befunde lassen sich auch auf die Schwangerschaft übertragen (Lukesch, 1981). Nur dass die zusätzliche angstauslösende Wirkung der Wehen fehlt.

\subsection{Fragestellungen und Hypothesen}

Die vorliegende Diplomarbeit soll den aus der Literatur (siehe oben) bekannten Zusammenhang zwischen Angst und Komplikationen stützen und ergänzen.

Verglichen werden Angstwerte von Frauen vor und nach der Geburt sowie die Rate an Komplikationen in der Schwangerschaft und während der Geburt. Dabei werden zwei Gruppen von Frauen verglichen: Die eine Gruppe setzt sich aus Frauen zusammen, die eine Hausgeburt planen, die andere setzt sich aus Frauen zusammen, die bewusst in einer Klinik entbinden.

Folgende Hypothesen werden überprüft:

Hypothese 1 prüft den Zusammenhang von Angst und Komplikationen. Hypothese 1a

Schwangere, bei denen Schwangerschaftskomplikationen auftreten, haben höhere Angstwerte als Schwangere mit komplikationsfreien Schwangerschaftsverläufen. Hypothese 1b

Frauen, bei denen Geburtskomplikationen auftreten, haben höhere Angstwerte als Frauen mit komplikationslosen Geburtsverläufen. 
Die Befunde in Abschnitt 2.4 zeigen, dass eine Hausgeburt nicht gefährlicher für Mutter und Kind ist als eine Geburt in einer Klinik, sogar sicherer ist, d.h. weniger Komplikationen unter der Geburt auftreten. Die vorliegende Diplomarbeit soll diese Ergebnisse bestätigen und somit als weitere empirische Evidenz für die Sicherheit der ausserklinischen Geburtshilfe dienen.

Diese Befunde werden mit folgender Hypothese überprüft:

\section{Hypothese 2}

Hypothese 2 prüft den Zusammenhang von Angst und geplanter Haus-/ Klinikgeburt.

H 0: Die Komplikationsrate bei Haus- und Klinikgeburten unterscheidet sich nicht

H 1: Eine geplante Hausgeburt ist mit weniger Komplikationen einhergehend als eine geplante Klinikgeburt.

Um die bestehende Literatur im Bereich „Angst in Abhängigkeit von der Entbindungsform" zu erweitern, greift die vorliegende Diplomarbeit dieses Thema auf und überprüft folgende Hypothese:

\section{Hypothese 3}

Hypothese 3 prüft den Zusammenhang von Angst und Endbindungsform.

Das allgemeine Angstniveau ist bei Frauen während der Schwangerschaft, vor und nach der Geburt, die eine Hausgeburt planen geringer als bei Frauen, die sich bewusst für eine Klinikgeburt entschieden haben. 


\section{Methode}

\subsection{Stichprobe}

Die Gesamtstichprobe der vorliegenden Arbeit umfasst $n=74$ Frauen. Davon hatten n=37 Frauen eine Hausgeburt geplant. Diese Frauen werden im Folgenden als Hausgeburtsfrauen bezeichnet. Diese Gruppe enthielt Frauen, die zwischen 22 und 44 Jahre alt $(M=31,73$ Jahre; $S D=4,84)$ waren.

$\mathrm{N}=37$ Frauen, die sich bewusst für eine Klinikgeburt entschieden hatten, werden im Folgenden als Klinikgeburtsfrauen bezeichnet. Die Frauen dieser Gruppe waren zwischen 24 und 39 Jahre alt ( $M=30,81$ Jahre; $S D=4,08)$. Alle Klinkgeburtsfrauen erfüllten auch die Kriterien, die für eine Hausgeburt notwendig sind (wie im Theorieteil ausgeführt und folgend). Das bedeutet, dass auch für sie eine Hausgeburt möglich gewesen wäre. Die Tabelle 2 zeigt einen Überblick der Stichprobe.

Tabelle 2: Stichprobe (Gruppenvergleich)

\begin{tabular}{|c|c|c|}
\hline & Klinikgeburtsfrauen & Hausgeburtsfrauen \\
\hline & $\begin{array}{l}\text { Frauen, die sich für eine } \\
\text { Klinikgeburt entschieden haben } \\
\qquad(\mathrm{n}=37)\end{array}$ & $\begin{array}{l}\text { Frauen, die eine Hausgeburt } \\
\text { geplant haben } \\
\qquad(\mathrm{n}=37)\end{array}$ \\
\hline Zeitpunkt der Erhebung & \multicolumn{2}{|c|}{$\begin{array}{l}\text { 1. Beginn Geburtsvorbereitungskurs } \\
\text { 2. Ende Geburtsvorbereitungskurs } \\
\text { 3. Geburtsbeginn } \\
\text { 4. Vier Wochen post partum (nach der Geburt) }\end{array}$} \\
\hline Ort der Erhebung & $\begin{array}{c}\text { Geburtsvorbereitungskurse } \\
\text { durch Hebammen in Waldshut- } \\
\text { Tiengen, Konstanz, Nagold und } \\
\text { Bietigheim }\end{array}$ & $\begin{array}{c}\text { Geburtsvorbereitungskurse durch } \\
\text { Hebammen in Konstanz, Rielasingen } \\
\text { und Taunusstein }\end{array}$ \\
\hline
\end{tabular}


Insgesamt wurden 152 Fragebogenpakete an Schwangere ausgegeben. Zwei Frauen lehnten eine Teilnahme ab (jeweils eine pro Gruppe).

Von den 47 verteilten Fragebögen in der Hausgeburtsgruppe kamen 42 zurück. Bei zwei Rückantworten war die namentliche Zuordnung nicht möglich und drei Exemplare waren unvollständig (es fehlte mehr als ein Fragebogen). Zwei Frauen konnten nicht mit in die Stichprobe aufgenommen werden, da sie vor Beendigung des Geburtsvorbereitungskurses geboren hatten (am Geburtstermin, keine Frühgeburten). Es gab damit 37 komplette Datensätze.

Von den 105 ausgegebenen Fragebogenpakete in der Klinikgeburtsgruppe kamen 39 zurück. Davon war ein Exemplar namentlich nicht zuzuordnen, eine Frau gebar in einem anderen Krankenhaus. 52 Frauen fielen heraus, da sie vor Beendigung des Geburtsvorbereitungskurses geboren hatten (am Geburtstermin, keine Frühgeburten). Somit wurden 37 komplette Datensätze erhalten.

Es gab keine Dropouts.

Zur Berechnung der Rücklauf-Quote wurde ein neuer Gesamtwert (ausgeteilter Bögen) berechnet. Frauen, die aufgrund einer Geburt vor Beendigung des Kurses, nicht teilnehmen konnten, gingen nicht mit in den Gesamtwert ein, um so bei der Rücklaufquote Unterschiede zwischen den Gruppen genauer beurteilen zu können. Siehe dazu Tabelle 3.

Tabelle 3: Rücklauf der Fragebögen

\begin{tabular}{l|c|c} 
Gruppe & Anzahl ausgeteilter Fragebögen & Rücklauf absolut/in Prozent \\
\hline Hausgeburt & 45 & $42 / 93,3 \%$ \\
\hline Klinikgeburt & 53 & $39 / 73,6 \%$ \\
\hline Gesamt & 98 & $81 / 82,7 \%$ \\
\hline
\end{tabular}




\subsection{Rekrutierung}

Um an der Untersuchung teilzunehmen, mussten die Frauen folgende Kriterien erfüllen:

B Teilnahme an einem Geburtsvorbereitungskurs

B Gesunde Frau

B Gesundes Kind

B Normaler Schwangerschaftsverlauf (zumindest bis Beginn der ersten Datenerhebung)

Ausgeschlossen wurden Frauen, die aufgrund der vom Bund Deutscher Hebammen e.V., dem Bund freiberuflicher Hebammen Deutschlands e.V. und dem Netzwerk zur Förderung der Idee Geburtshäuser in Deutschland e.V. (2000) empfohlenen und veröffentlichten Auswahlkriterien, nicht für eine Hausgeburt geeignet waren. Das bedeutet, dass alle Frauen, die an der Studie teilnahmen, eine Hausgeburt hätten anstreben können.

Die einzelnen Negativkriterien, die gegen eine Hausgeburt sprechen, werden im Folgenden detailliert aufgelistet (gemäss der Empfehlungen und Auswahlkriterien für die Wahl des Geburtsortes oben genannter Berufsverbände, 2000):

B Allgemeine Erkrankungen

- Epilepsie mit Dauermedikation

- Insulinpflichtiger Diabetes mellitus

- HIV

B Besonderheiten in der geburtshilflichen Anamnese

- Zustand nach Uterusruptur

- Zustand nach Re-Sectio ohne vaginale Geburt

- Zustand nach vorzeitiger Plazentalösung

- Zustand nach hohem postpartalem Blutverlust 
B Krankheiten und Besonderheiten in der jetzigen Schwangerschaft

Anämie mit klinischen Symptomen

- Gerinnungsstörungen

- Heroin- bzw. Methadonabhängigkeit

- E-P-H-Gestose, HELLP-Syndrom

- Gemini in ungünstiger Lage und Drillinge

- Vorzeitiger Blasensprung vor der 37. Schwangerschaftswoche (SSW)

B Besonderheiten während der Geburt

- Verdacht auf Amnioninfektionssyndrom

- Geburt vor der 37. SSW

- Anzeichen für fetale Asphyxie in der Eröffnungsperiode (EP), ggf. auch Austreibungsperiode (AP)

- Mehr als mensesstarke Blutung in der EP

- Verdacht auf vorzeitige Plazentalösung

- Beckenendlage und protrahierter Verlauf

- Vorzeitiger Blasensprung ohne Wehentätigkeit mit deutlichem Farbwechsel des Fruchtwassers

- Blutungen in der Plazentar- und/oder Nachgeburtsperiode, die mit den Mitteln der Hebamme nicht zu beheben sind

- Unvollständige Plazenta

Für beide Gruppen mussten obige Kriterien gelten, damit die Gruppen bezüglich Gesundheit (von Mutter und Kind) und Geburtsvorbereitung homogen sind und somit vergleichbar sind.

\subsection{Durchführung}

Die Datenerhebung fand von Januar 2001 bis Oktober 2002 statt.

Die Daten der Klinikgeburtsfrauen wurden von mir selbst und in Waldshut-Tiengen zusätzlich auch von den kursleitenden Hebammen erhoben. Die Daten der 
Hausgeburtsfrauen wurden ebenfalls von mir selbst und in den Konstanzer Kursen ausserdem von den kursleitenden Hebammen erhoben. Die Datenerhebung fand am ersten und letzten Abend des Geburtsvorbereitungskurses, bei Geburtsbeginn (Wehen und/oder Blasensprung) und vier Wochen nach der Geburt statt.

Die Teilnahme an der Studie erfolgte freiwillig und unentgeltlich. Vorab bekamen die Teilnehmerinnen ein Informationsblatt über die Untersuchung. Die ersten Fragebögen wurden in meiner Anwesenheit oder der kursleitenden Hebamme ausgefüllt, so dass die Möglichkeit zu Rückfragen bestand. Dies war aber auch immer telefonisch bei mir möglich. Die Anleitung zum Ausfüllen der Fragebögen war in schriftlicher Form standardisiert und wurde bei der ersten Datenerhebung auch mündlich mitgeteilt. Das Ausfüllen aller Fragebögen insgesamt dauerte ca. 20-25 Minuten. Zum einen erfolgte die Rückgabe der Fragebögen direkt vor Ort an mich, zum anderen war den Fragebögen ein frankierter Rückumschlag angeheftet. Ein Muster der gesamten Unterlagen und Fragebögen für die Untersuchung befindet sich im Anhang B. Bei Interesse seitens der Teilnehmerin, wurde eine Zusammenfassung der Hauptergebnisse in Aussicht gestellt.

Als Erhebungsinstrumente dienten verschiedene Fragebögen. Sie wurden immer in der folgenden Reihenfolge vorgelegt:

B Erster Kursabend

- State-Trait-Angstinventar (STAI) Form X 1 (State-Angst Zeitpunkt 1: X1,1)

- STAI Form X 2 (Trait-Angst Zeitpunkt 1: X2,1)

- Soziodemographische Fragen (Datenblatt)

B Letzter Kursabend

STAI X 1 (Zeitpunkt 2: X1,2)

B Geburtsbeginn

STAI X 1 (Zeitpunkt 3: X1,3) 
B Vier Wochen nach Geburt

STAI X 1 (Zeitpunkt 4: X1,4)

- STAIX 2 (Zeitpunkt 2: X2,2)

Zusätzlich wurden bei allen Frauen Daten zur Anamnese, zum Schwangerschaftsund Geburtsverlauf aus der Patientenakte erhoben.

\subsection{Verwendete Verfahren und Variablenbildung}

\subsubsection{Soziodemographische und schwangerschaftsspezifische Fragen}

Erfasst wurden soziodemographische Daten und Angaben zur persönlichen Situation wie Alter, Familienstand, Schulabschluss, momentane Berufstätigkeit, Anzahl der Schwangerschaften (Gravidität) und Anzahl der Geburten (Parität). Anhand der Gravidität und Parität (sowie Anamnese), konnten Fehlgeburten und die Anzahl bereits geborener Kinder erschlossen werden.

Die Fragen befinden sich im Anhang B unter der Überschrift „Datenblatt“.

\section{Variablenbildung}

Mit den soziodemographischen und schwangerschaftsspezifischen Daten wurden keine Hypothesen geprüft. Berechnet wurden Einzelvergleiche zwischen den Gruppen auf der Ebene der Items. Zudem wurden diese Daten in die explorativen Analysen miteinbezogen.

\subsubsection{Fragebogen zur Erfassung der Angst}

Zur Messung der Angst wurde die auf dem "State-Trait Anxiety Inventory" von Spielberger, Gorsuch \& Lushene (1970) basierende deutsche Adaption, das State-Trait-Angstinvetar (STAI), verwendet (Laux, Glanzmann, Schaffner \& Spielberger, 1981). Die zwei Skalen des STAI mit jeweils 20 Items dienen der Erfassung von Angst als Zustand (State-Angst), d.h. wie die Person sich jetzt in diesem Moment fühlt, und Angst als Eigenschaft (Trait-Angst), d.h. wie die Person 
sich im allgemeinen fühlt. Die Beantwortung erfolgt auf einer vierstufigen Skala mit Häufigkeitsangabe: (1) überhaupt nicht, (2) ein wenig, (3) ziemlich, (4) sehr.

Ziel der Fragebogenkonstruktion von Spielberger et al. (1970) war die differenzierte Erfassung von Angst als Zustand und Angst als Eigenschaft wie sie von Cattell \& Scheier (1961) unterschieden wurde.

Die Versuchspersonen wurden durch die Standardinstruktion angeleitet.

Die Fragebögen befinden sich im Anhang B unter der Überschrift „Fragebogen zur Selbstbeschreibung" (STAI-G Form X 1 und X 2).

Ausschlaggebend für die Wahl dieses Instruments war, dass sowohl die Zustandsangst (State) als auch Angst als Persönlichkeitsmerkmal (Trait) erfasst werden kann. Aufgrund der Messung bei Geburtsbeginn kann somit die Angst vor der Geburt erfasst werden, wenn man davon ausgeht, dass die Situationsveränderung (bevorstehende Geburt) ausschlaggebend für die momentane Zustandsbeurteilung ist. Durch die Erfassung der Trait-Angst prä- und postpartal, kann eine eventuelle Variation der Angst als Eigenschaft festgestellt werden (Einfluss der Geburt).

Die Entscheidung, Angst (State und Trait) erst im dritten Trimenon zu erheben, beruhte auf hauptsächlich praktischen Gründen (z.B. Verfügbarkeit der Frauen). Der Erhebungszeitraum bis zur Geburt deckt sich ungefähr mit der letzten Schwangerschaftsphase gemäss dem Phasenmodell nach Gloger-Tippelt (1988). Nach diesem Modell verläuft Angst diskontinuierlich. In dieser Phase sind Ängste wieder stärker.

\section{Variablenbildung}

Zur Ermittlung der Rohwerte der einzelnen Untersuchungsteilnehmerinnen wurden für beide Skalen getrennt die Summenwerte der jeweils 20 Feststellungen errechnet. Beide Skalen können die Werte von minimal 20 (Nichtvorhandensein) bis maximal 80 (maximale Intensität) erreichen. 
Folgende Werte beziehen sich auf die Eichstichprobe ( $\mathrm{n}=1278$ Frauen) von 1977 (Laux, L. et al.1981). Der Mittelwert beträgt 37,01, die Standardabweichung 9,95, Cronbach's Alpha 0,91 und die Schiefe 0,61 für die Trait-Angst. Für die StateAngst ergeben sich folgende Werte, $M=38,08, S D=10,29$, Cronbach's Alpha 0,91 und Schiefe 0,81.

\subsubsection{Erfassung von Komplikationen}

Die Erfassung von Schwangerschafts- und Geburtskomplikationen erfolgte über die Einsicht in die Patientenakten. Es wurden solche Komplikationen erfasst, die im Theorieteil ausgeführt worden sind.

\section{Variablenbildung}

Die Daten der vorliegenden Arbeit wurden dichotomisiert, d.h. es wurde erfasst, ob es Komplikationen gab oder nicht.

In einem zweiten Schritt wurden drei Kategorien von Komplikationen den Geburtsverlauf betreffend gebildet:

1. Komplikationen, die den zeitlichen Verlauf einer Geburt betreffen (z.B. Wehenschwäche, Geburtsstillstand, protrahierter Geburtverlauf)

2. Komplikationen in der Plazentarperiode (Plazentalösungsstörungen, Blutungen)

3. Sonstige (Blutung in der Eröffnungsperiode, pathologisches Cardiotokogramm und Uterusruptur)

Ausserdem wurden Mehrfachkomplikationen (mehr als eine Komplikation) beim Geburtsverlauf (ja/nein) erfasst.

\subsection{Datenanalyseverfahren}

Alle statistischen Verfahren wurden mit Hilfe des Statistikprogramms SPSS ${ }^{\circledR} 11,0$ durchgeführt. Für alle Analysen wurde ein Signifikanzniveau von $\alpha=5 \%$ festgesetzt. Die Prüfung auf Signifikanz erfolgte grundsätzlich zweiseitig. 
Aufgrund der Stichprobengrösse von $n=74$ wurde von der Gültigkeit des zentralen Grenzwerttheorems ausgegangen: „Die Verteilung von Mittelwerten aus Stichproben des Umfanges n, die sämtlich derselben Grundgesamtheit entnommen wurden, geht mit wachsendem Stichprobenumfang in eine Normalverteilung über" (Bortz, 1999, S. 93). Deshalb wurden die Daten nicht auf Normalverteilung geprüft, die als Voraussetzung für die Durchführung der angewandten Tests gilt.

Bei den durchgeführten $\mathrm{t}$-Tests für unabhängige Stichproben bestand Varianzgleichheit, was der Levene-Test zeigte.

Die Voraussetzung für die Durchführung von Varianzanalysen mit Messwiederholung, Gleichheit der Kovarianzmatrizen, wurde durch den Box-Test überprüft, bei mehr als zwei Messzeitpunkten wurde zusätzlich der Mauchly-Test zur Überprüfung der Sphärizität verwendet. Sofern bei den einzelnen Analysen in Kapitel 5 nichts anderes genannt wurde, waren die oben genannten Voraussetzungen erfüllt. Bei Nichterfüllung wurde dies speziell erwähnt, wobei der geplante Test trotzdem durchgeführt wurde, da die Verfahren ziemlich robust gegenüber Verletzungen der Verteilungsannahme sind (Havlicek \& Peterson, 1974; zit. nach Bortz, 1999).

Die Voraussetzung der Kolinearität für die Durchführung von linearen und binär logistischen Regressionen wurde bei allen durchgeführten Analysen dieser Art geprüft. Die Kolinearitätsdiagnose erfolgte über den VIF, der nicht grösser als drei sein sollte (Chatterjee \& Hadi, 1988). Es wurden nur bei erfüllter Voraussetzung Regressionen berechnet.

\subsubsection{Berechnung von Gruppenunterschieden}

Mit den soziodemographischen und schwangerschaftsspezifischen Daten wurden Gruppenvergleiche zwischen den Haus- und Klinikgeburtsfrauen berechnet. Verwendet wurden hierfür t-Tests für unabhängige Stichproben und $\chi_{-}$-Tests.

Zur Berechnung des Zusammenhangs zwischen Angst und Komplikationen (Hypothese 1 b) sowie Angst und Entbindungsform (Hypothese 3) wurden die 
vorliegenden Daten zweifaktoriellen Varianzanalysen mit Messwiederholungen unterzogen, um Mittelwertsunterschiede zwischen den Gruppen zu berechnen.

Zur Überprüfung, ob Unterschiede bezüglich der Komplikationsrate (Hypothese 2) sowie Komplikationskategorien und Entbindungsform bestehen, wurde der $\chi_{-}$-Test für unabhängige Stichproben gewählt. Um Unterschiede zwischen den beiden untersuchten Gruppen bezüglich Mehrfachkomplikationen festzustellen, wurde der Fisher's Exact Test angewandt.

So konnte festgestellt werden, ob sich die Stichproben in den erhobenen Massen unterschieden.

\subsubsection{Weitere durchgeführte Untersuchungen (explorativer Art)}

Die State- und Trait-Angst (unabhängig von den Gruppen) und der Einfluss der Parität wurden mittels zweifaktorieller Varianzanalysen mit Messwiederholung (und Kontrastberechnung) untersucht. So konnten signifikante Unterschiede bezüglich der Angst als Gesamtmittelwert über alle Messzeitpunkte und zwischen den einzelnen Zeitpunkten ermittelt werden sowie Unterschiede zwischen Erst- und Mehrgebärenden und Interaktionen.

Interaktionen wurden festgestellt, in dem man mittels mehrfaktoriellen Varianzanalysen mit Messwiederholung, Mittelwertsunterschiede zwischen den Gruppen berechnete.

Um Vorhersagen von Zusammenhängen zu ermitteln, wurden binär logistische Regressionsanalysen und lineare Regressionsanalysen verwendet.

Im Anhang A befinden sich weitere Analysen. Aufgrund ihrer (meist) nicht signifikanten Ergebnisse sowie ökonomischer Gründe wurden sie im Kapitel 4 nicht erwähnt. Es wurden keine weiteren Verfahren verwendet, ausser für das Zusammenhangsmass zwischen den State- und Trait-Messungen. Hierzu wurden Korrelationskoeffizienten nach Pearson berechnet. 
Details zu den verwendeten Verfahren sind in entsprechenden Statistikbüchern zu finden (z.B. Bortz, 1999). 


\section{Ergebnisse}

\subsection{Beschreibung der Stichprobe}

\subsubsection{Soziodemographische und schwangerschaftsspezifische Daten}

Vor der Überprüfung der Hypothesen wurden die soziodemographischen Daten der Haus- und Klinikgeburtsfrauen miteinander verglichen. Dies war in erster Linie notwendig, um die Homogenität der beiden Gruppen zu prüfen. Homogenität ist die Voraussetzung, damit die Gruppen miteinander verglichen werden können. Ferner kann man bei auftretenden Unterschieden zwischen den beiden Gruppen überprüfen, ob die gefundenen Unterschiede charakteristisch für die Gruppe der Hausgeburtsfrauen bzw. der Klinikgeburtsfrauen sind, d.h. ob sie ein spezifisches Merkmal für die jeweilige Gruppe darstellen. Schwangerschaftsspezifische Daten wurden zwischen den beiden Gruppen verglichen, um vor der Überprüfung der Hypothesen mögliche Drittvariablen zu identifizieren.

Die Tabellen 4, 5, 6 und 7 geben einen Überblick über die soziodemographischen und schwangerschaftsspezifischen Daten der beiden Gruppen.

Tabelle 4: Vergleich von Alter und Kinder zwischen den Gruppen (t-Tests)

\begin{tabular}{lcccc}
\hline & $\begin{array}{c}\text { Hausgeburtsfrauen } \\
\ldots \ldots \ldots(\mathrm{n}=37) \\
\mathrm{m}(\mathrm{sd})\end{array}$ & $\begin{array}{c}\text { Klinikgeburtsfrauen } \\
(\mathrm{n}=37) \\
\mathrm{m}(\mathrm{sd})\end{array}$ & $\mathrm{df}$ & t-Wert \\
\hline Alter in Jahren & $31,73(4,84)$ & $30,81(4,08)$ & 72 & $-0,883+$ \\
Anzahl der Kinder & $1,03(0,897)$ & $0,81(1,151)$ & 72 & $-0,901+$ \\
\hline$+p>0,05$ & & & &
\end{tabular}

Das Alter der Hausgeburtsfrauen betrug im Durchschnitt 31,73 Jahre (sd=4,84) und das Alter der Klinikgeburtsfrauen 30,81 Jahre $(s d=4,08)$. Der durchgeführte t- 
Test für unabhängige Stichproben ergab keinen signifikanten Unterschied (siehe Tabelle 4).

Die Hausgeburtsfrauen hatten im Durchschnitt bereits 1,03 Kinder $(s d=0,897)$ und die Klinikgeburtsfrauen 0,81 Kinder $(s d=1,51)$. Der durchgeführte t-Test für unabhängige Srichproben ergab keinen signifikanten Unterschied für die Anzahl bereits geborener Kinder wie aus Tabelle 4 ersichtlich.

Tabelle 5: Vergleich des Familienstands zwischen den Gruppen ( $\chi_{-}$-Test)

\begin{tabular}{lcc}
\hline & $\begin{array}{c}\text { Hausgeburtsfrauen } \\
(\mathrm{n}=36)\end{array}$ & $\begin{array}{c}\text { Klinikgeburtsfrauen } \\
(\mathrm{n}=34)\end{array}$ \\
\hline ledig & $12(32,4 \%)$ & $4(10,8 \%)$ \\
verheiratet/geschieden & $22(59,5 \%)$ & $28(75,7 \%)$ \\
\hline
\end{tabular}

$\chi_{-}(\mathrm{LR})=4,797^{*} ; \mathrm{df}=1$

${ }^{*} \mathrm{p}<0,05$

Bezüglich des Familienstandes erbrachte der durchgeführte einen signifikanten Unterschied zwischen den beiden Gruppen. In der Hausgeburtgruppe waren signifikant mehr ledige Frauen. Da bei der Analyse mit den Kategorien „ledig“, „verheiratet" und "geschieden“ zwei Zellen eine erwartete Häufigkeit kleiner 5 hatten, wurden Kategorien zusammengefasst, damit die Voraussetzungen für den $\chi \_$-Test erfüllt waren (bei nicht dichotomen Variablen müssen die Erwartungswerte der Zellen grösser als 5 sein). Die genauen Ergebnisse sind Tabelle $5 \mathrm{zu}$ entnehmen. 
Tabelle 6: Vergleich von Bildungsniveau zwischen den Gruppen ( $\left.\chi_{-}-T e s t\right)$

\begin{tabular}{lll}
\hline & $\begin{array}{l}\text { Hausgeburtsfrauen } \\
(\mathrm{n}=37)\end{array}$ & $\begin{array}{l}\text { Klinikgeburtsfrauen } \\
(\mathrm{n}=37)\end{array}$ \\
\hline Hauptschulabschluss/Mittlere Reife & $13(35,1 \%)$ & $26(70,3 \%)$ \\
Fachhochschul-/Hochschulreife & $10(27,0 \%)$ & $8(21,6 \%)$ \\
Fachhochschul-/Hochschulabschluss & $14(37,8 \%)$ & $3(8,1 \%)$ \\
\hline
\end{tabular}

$\chi_{-}(\mathrm{LR})=12,363^{*}, \mathrm{df}=2$

${ }^{*} \mathrm{p}<0,05$

Zum Bildungsniveau der Frauen wurde ein $\chi_{-}$-Test zwischen den beiden Gruppen über die drei Niveaus durchgeführt. Zwischen den drei Niveaus wurden keine Einzelvergleiche berechnet. Auch hier wurden Kategorien zusammengefasst (Gründe siehe oben). Das Bildungsniveau der Hausgeburtsfrauen lag signifikant höher als bei den Klinikgeburtsfrauen. Die genauen Ergebnisse zeigt Tabelle 6.

Tabelle 7: Vergleich von Berufstätigkeit zwischen den Gruppen ( $\left.\chi_{-}-T e s t\right)$

\begin{tabular}{cc}
\hline Hausgeburtsfrauen & Klinikgeburtsfrauen \\
$(\mathrm{n}=37)$ & $(\mathrm{n}=37)$ \\
\hline
\end{tabular}

Berufstätigkeit während Schwangerschaft

$\chi_{-}(\mathrm{LR})=0,922+; \mathrm{df}=1$

$+p>0,05$

In bezug auf die Berufstätigkeit während der Schwangerschaft zeigten sich keine signifikanten Gruppenunterschiede. Berechnet wurden zum Gruppenvergleich ein $\chi \_$-Test. Die genauen Ergebnisse sind in Tabelle 7 dargestellt. 
Tabelle 8: Vergleich von Fehlgeburten zwischen den Gruppen ( $\chi_{-}$-Test)

\begin{tabular}{lcc}
\hline & $\begin{array}{c}\text { Hausgeburtsfrauen } \\
(\mathrm{n}=37)\end{array}$ & $\begin{array}{c}\text { Klinikgeburtsfrauen } \\
(\mathrm{n}=37)\end{array}$ \\
\hline Vorausgegangene Fehlgeburten & $8(21,6 \%)$ & $4(10,8 \%)$ \\
\hline$\chi_{-}(\mathrm{LR})=1,617+; \mathrm{df}=1$ & & \\
$+\mathrm{p}>0,05$ & &
\end{tabular}

Es zeigten sich keine signifikanten Gruppenunterschiede bezüglich vorausgegangener Fehlgeburten. Zum Gruppenvergleich wurde ein $\chi_{-}$-Test berechnet. Die genauen Ergebnisse sind in Tabelle 8 aufgeführt.

\subsection{Angst und Komplikationen}

\subsubsection{Zusammenhang von Angst und Schwangerschafts- komplikationen}

Die Hypothese 1 a konnte aufgrund der vorliegenden Daten nicht überprüft werden, da nur eine Studienteilnehmerin eine Schwangerschaftskomplikation aufwies.

\subsubsection{Zusammenhang von Angst und Geburtskomplikationen}

Hypothese $1 \mathrm{~b}$ diente zur Überprüfung, ob es einen Zusammenhang zwischen Angst und Geburtskomplikationen gibt. Die durchgeführte zweifaktorielle Varianzanalyse mit Messwiederholung erbrachte keinen signifikanten Zusammenhang zwischen Geburtskomplikationen und Angstwerten (Mittelwert über alle Messzeitpunkte). Dies galt sowohl für die State-Angst als auch TraitAngst. Somit konnte Hypothese $1 \mathrm{~b}$ nicht bestätigt werden. Demnach hatten 
Frauen mit Geburtskomplikationen keine signifikant höheren Angstwerte. Die genauen Ergebnisse sind in Tabelle 9 dargestellt. Der Box-Test bei der State-Angst-Analyse war signifikant, weshalb die Gleichheit der Kovarianzmatrizen nicht gegeben war $(F(10,9269)=2,934 ; p<0,05)$.

Tabelle 9: Ergebnisse der Varianzanalysen von State-/Traitangst und Geburtskomplikationen

\begin{tabular}{l|c|c|c|c|c} 
Effekt & $\begin{array}{c}\text { Wilks } \\
\text { Lambda }\end{array}$ & $\mathrm{F}$ & $\mathrm{df}_{1}$ & $\mathrm{df}_{2}$ & Signifikanz \\
\hline $\begin{array}{l}\text { State-Angst } \\
\text { Komplikation }\end{array}$ & 0,989 & 0,241 & 3 & 65 & 0,867 \\
\hline $\begin{array}{l}\text { Trait-Angst } \\
\text { Komplikation }\end{array}$ & -- & 0,618 & 1 & 69 & 0,435 \\
\hline
\end{tabular}

In einem zweiten Schritt wurde untersucht, ob sich die Ergebnisse ändern, wenn die Komplikationen kategorial in die Analysen eingehen.

Einen signifikanten Zusammenhang ergab die zweifaktorielle Varianzanalyse mit Messwiederholung zwischen der State-Angst und der Kategorie 3 (sonstige Komplikationen). Für die Trait-Angst konnte dieser Zusammenhang nicht gezeigt werden (Gleichheit der Kovarianzmatrizen nicht gegeben: Box-Test $F(3,1310)=2,636 ; p<0,05)$.

Frauen mit Komplikationen der Kategorie 3 hatten signifikant höhere StateAngstwerte (Gesamtmittelwert über die vier Messungen) als die ohne. Zu den einzelnen Messzeitpunkten bestanden keine signifikanten Unterschiede zwischen den Frauen mit und ohne Komplikationen der Kategorie 3, welches die dazu berechneten t-Tests für unabhängige Stichproben zeigten. Das Ergebnis war schon ohne Bonferoni-Korrektur nicht signifikant. In Tabelle 10 und 11 sowie Abbildung 2 werden die genauen Ergebnisse der Varianzanalyse und t-Tests gezeigt. Die Analysen mit den anderen Kategorien befinden sich im Anhang A. 
Tabelle 10: State- bzw. Trait-Angst und Komplikationen der Kategorie 3 (Varianzanalyse)

\begin{tabular}{l|c|c|c|c|c} 
Effekt & Wilks Lambda & $\mathrm{F}$ & $\mathrm{df}_{1}$ & $\mathrm{df}_{2}$ & Signifikanz \\
\hline State-Angst*Komplikationen & 0,870 & 3,248 & 3 & 65 & 0,027 \\
\hline Trait-AngstKomplikationen & -- & 0,612 & 1 & 69 & 0,437 \\
\hline
\end{tabular}

Tabelle 11: Vergleich der State-Angst zu den verschiedenen Messzeitpunkten zwischen den Gruppen mit und ohne Komplikationen der Kategorie 3 (t-Test)

\begin{tabular}{l|c|c|c} 
& $\mathrm{t}$-Wert & df & Signifikanz \\
\hline STAI X1,1 & 1,233 & 72 & 0,225 \\
\hline STAI X1,2 & $-1,455$ & 69 & 0,150 \\
\hline STAI X1,3 & $-1,297$ & 70 & 0,199 \\
\hline STAI X1,4 & 0,495 & 72 & 0,622 \\
\hline
\end{tabular}

Abbildung 2: Verlauf der State-Angst und Komplikationen (Kategorie 3)

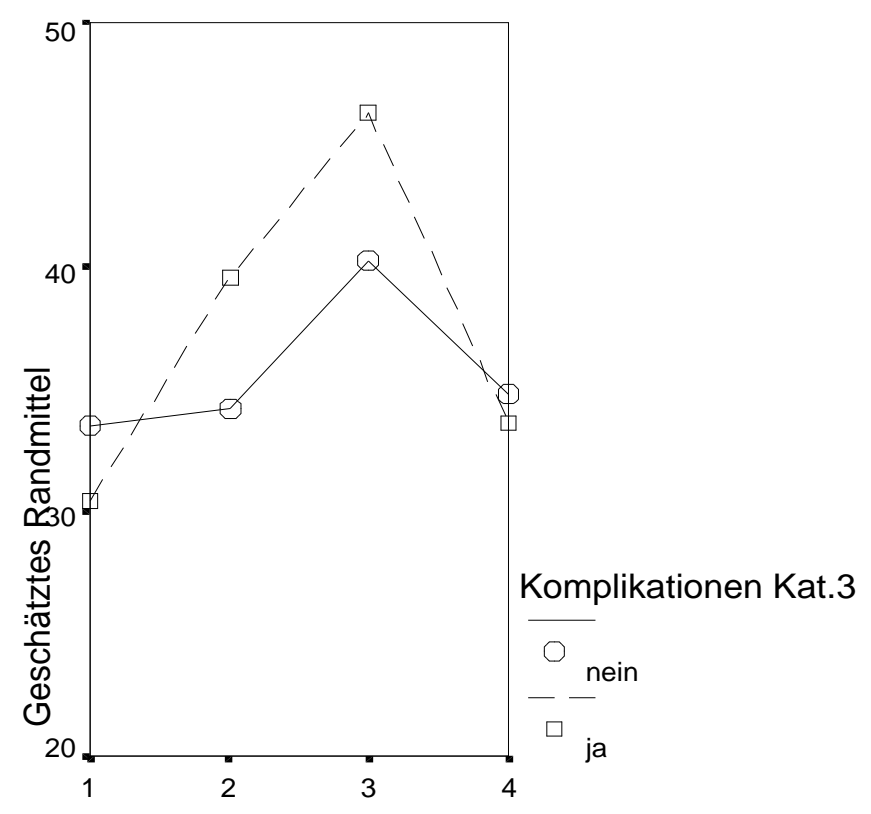

Messzeitpunkte 


\subsection{Zusammenhang zwischen Komplikationen und Entbindungsform}

Hypothese 2 diente der Prüfung des Zusammenhangs zwischen Angst und geplanter Haus-/Klinikgeburt. Hypothese $2(\mathrm{H} 0)$ postulierte, dass sich geplante Haus- und Klinikgeburten bezüglich ihrer Komplikationsrate nicht unterscheiden. Zwischen den beiden Gruppen ergaben sich keine signifikanten Unterschiede bezüglich der Anzahl an Komplikationen, wie der dazu durchgeführte $\chi_{-}$-Test zeigte. Damit bewährte sich die Hypothese $2(\mathrm{H} 0)$, das heisst, eine geplante Hausgeburt wies keine höhere Komplikationsrate als eine geplante Klinikgeburt auf, aber auch keine niedrigere Rate (Alternativhypothese $\mathrm{H} 1$ verworfen). Die genauen Ergebnisse sind in Tabelle 12 dargestellt.

Tabelle 12: Komplikationen und Entbindungsform ( $\chi_{-}-$Test $)$

\begin{tabular}{l|c|c} 
& \multicolumn{2}{|c}{$\begin{array}{c}\text { Komplikationen } \\
\text { Anzahl (\%) }\end{array}$} \\
\hline Hausgeburt & nein & ja \\
\hline Klinikgeburt & $27(73,0 \%)$ & $10(27,0 \%)$ \\
\hline
\end{tabular}

$\chi_{-}(\mathrm{LR})=2,149+; \mathrm{df}=1$

$+p>0,05$

In einem zweiten Schritt wurde untersucht, ob sich Unterschiede zwischen den Gruppen bezüglich der Komplikationsrate ergeben, wenn die Komplikationen kategorial in die Analyse einflossen. Der dazu berechnete $\chi_{-}$-Test zeigte einen signifikanten Gruppenunterschied: die Kategorie 2 (Komplikationen in der Plazentarperiode) trat signifikant häufiger in der Gruppe der Klinikgeburtsfrauen auf, d.h. bei Frauen, die geplant in der Klinik geboren hatten, traten häufiger Komplikationen in der Plazentarperiode auf als bei Frauen, die eine Hausgeburt geplant hatten. Die genauen Ergebnisse sind in Tabelle 13 und Abbildung 3 dargestellt. Im Anhang A sind die Analysen der weiteren Kategorien zu finden. 
Tabelle 13: Komplikationen (Kategorie 2) und Entbindungsform ( $\chi_{-}$-Test)

\begin{tabular}{l|c|c} 
& \multicolumn{2}{|c}{ Komplikationen in der Plazentarperiode Anzahl (\%) } \\
\hline Klinikgeburt & nein & ja \\
\hline Hausgeburt & $26(41,3 \%)$ & $11(100 \%)$ \\
\hline
\end{tabular}

$\chi_{-}(\mathrm{LR})=17,18^{*} ; \mathrm{df}=1$

${ }^{*} p<0,001$

Abbildung 3: Komplikationen (Kategorie 2) und Entbindungsform

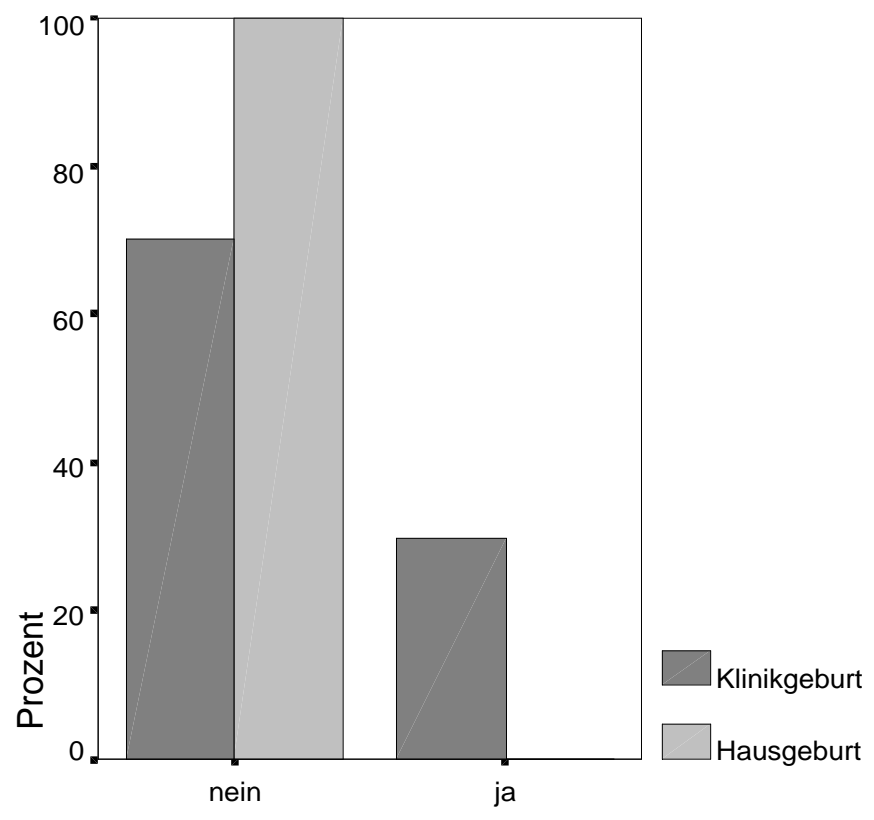

Komplikationen Kat. 2

Des weiteren wurden Mehrfachnennungen der Komplikationskategorien in die Analysen miteinbezogen. Dabei berechnete der Fisher's Exact Test einen signifikanten Zusammenhang zwischen geplanter Klinikgeburt und Mehrfachkomplikationen. Folglich hatten Frauen, die geplant in der Klinik geboren hatten, häufiger mehr als nur eine Komplikation als Frauen, die eine Hausgeburt 
geplant hatten. Die Ergebnisse sind in Tabelle 14 und Abbildung 4 detailliert dargestellt.

Tabelle 14: Mehrfachkomplikationen und Entbindungsform (Fisher's Exact Test)

\begin{tabular}{l|c|c} 
& \multicolumn{2}{|c}{$\begin{array}{c}\text { Mehrfachkomplikationen } \\
\text { Anzahl (\%) }\end{array}$} \\
\hline & ja & nein \\
\hline Hausgeburt & $1(9,1 \%)$ & $9(60 \%)$ \\
\hline Klinikgeburt & $10(90,9 \%)$ & $6(40 \%)$ \\
\hline
\end{tabular}

$\chi_{-}(\mathrm{LR})=7,754^{*} ; \mathrm{df}=1$

${ }^{*} \mathrm{p}<0,05$

Abbildung 4: Mehrfachkomplikationen und Entbindungsform

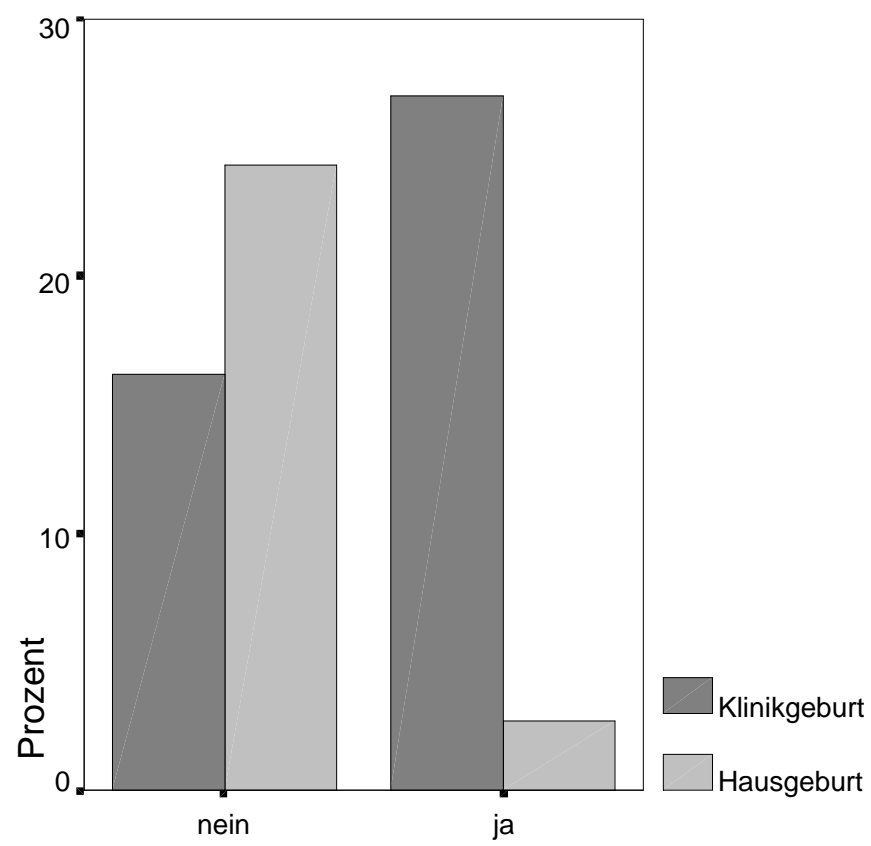

Mehrfachkomplikationen

\subsection{Zusammenhang zwischen Angst und Entbindungsform}

In Hypothese 3 ging man davon aus, dass Frauen, die eine Hausgeburt planen, während der Schwangerschaft, vor und nach der Geburt, weniger ängstlich sind als jene, die eine Klinikgeburt planen. Die untersuchten Gruppen unterschieden sich 
nicht in bezug auf die Angstwerte (Mittelwerte). Dies galt sowohl für die StateAngst als auch für die Trait-Angst, wie die durchgeführten zweifaktoriellen Varianzanalysen mit Messwiederholungen zeigten. Der Unterschied bezüglich der State-Angst zwischen den Gruppen lag nur knapp über dem 5\%-Signifikanzniveau $(0,06)$. Somit bestand der Trend, dass Hausgeburtsfrauen weniger ängstlich (State-Angst) waren als Klinikgeburtsfrauen. Die Hypothese 3 konnte jedoch nicht bestätigt werden. Demnach waren Hausgeburtsfrauen während der Schwangerschaft, vor und nach der Geburt nicht weniger ängstlich als Klinikgeburtsfrauen. Es konnte kein Zusammenhang zwischen Angst und geplanter Haus- bzw. Klinikgeburt gezeigt werden. Die Gleichheit der Kovarianzmatrizen war nicht gegeben, was der Box-Test zeigte $(F(10,21078)=2,854 ; p<0,05)$. Tabelle 15 und Abbildung 5 zeigen die genauen Ergebnisse.

Tabelle 15: State- und Traitangst und Entbindungsform (Varianzanalyse)

\begin{tabular}{l|c|c|c|c|c}
\multicolumn{1}{c|}{ Effekt } & Wilks Lambda & $\mathrm{F}$ & $\mathrm{df}_{1}$ & $\mathrm{df}_{2}$ & Signifikanz \\
\hline $\begin{array}{l}\text { State-Angst } \\
\text { Entbindungsform }\end{array}$ & 0,894 & 2,581 & 3 & 65 & 0,061 \\
\hline $\begin{array}{l}\text { Trait-Angst } \\
\text { Entbindungsform }\end{array}$ & -- & 0,989 & 1 & 69 & 0,324 \\
\hline
\end{tabular}




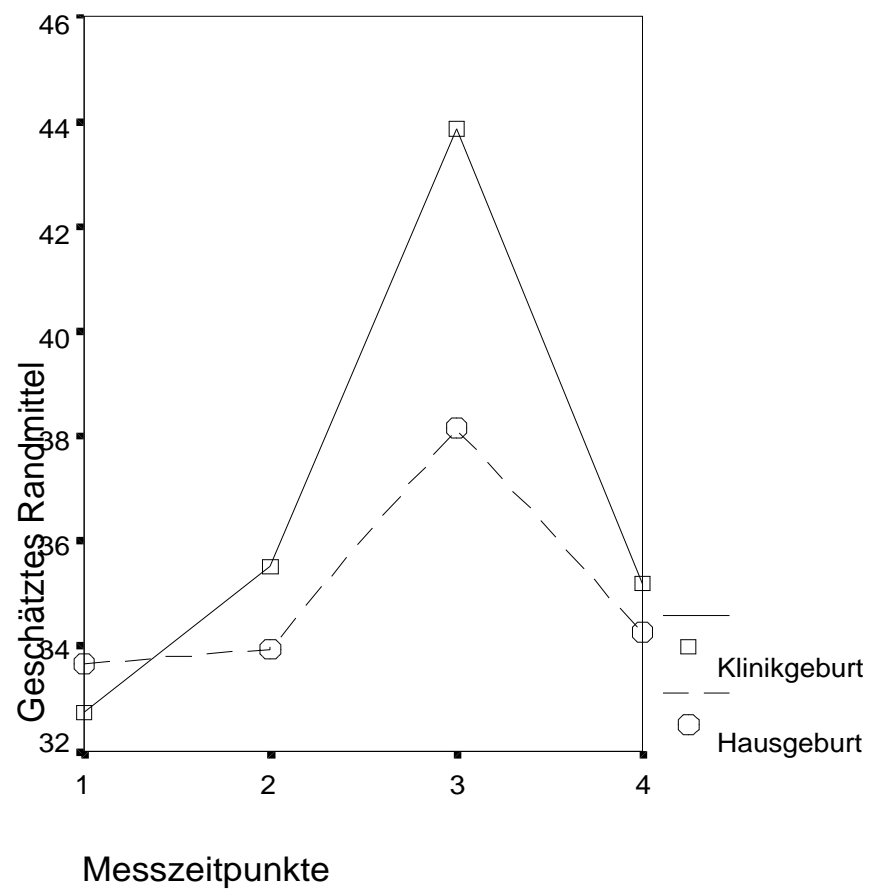

\subsection{Weitere durchgeführte Untersuchungen}

In diesem Abschnitt werden weitere, explorative Analysen beschrieben. Es werden (überwiegend) nur Untersuchungsergebnisse genannt, die signifikant waren. Alle weiteren durchgeführten Analysen sind im Anhang A zu finden.

\subsubsection{State- und Trait-Angst (unabhängig von Entbindungsform)}

\subsubsection{State-Angst (abhängig und unabhängig von Parität)}

Der berechnete Mittelwertsunterschied der State-Angst über die vier Messzeitpunkte war signifikant, unabhängig von der Gruppenzugehörigkeit (Hausbzw. Klinikgeburt). Zwischen dem Angstwert des dritten Messzeitpunkts (bei Geburtsbeginn) und allen drei anderen (zu Beginn des Geburtsvorbereitungskurses, Ende Geburtsvorbereitungskurs und vier Wochen nach Geburt) ergaben sich signifikante Unterschiede (durch zusätzliche Kontrastberechnungen). Die durchgeführte zweifaktorielle Varianzanalyse mit Messwiederholung ergab keinen weiteren signifikanten Haupteffekt. Der Mittelwertsunterschied (der vier Messzeitpunkte) bezüglich der State-Angst zwischen Erstgebärenden und Mehrgebärenden war demnach nicht signifikant. Auch die Interaktion zwischen Zeit (Messzeitpunkte) und Parität (Erst- und 
Mehrgebärende) war nicht signifikant. Siehe dazu Tabelle 16 sowie 17 und Abbildung 6.

Tabelle 16: Haupteffekte und Interaktion (State-Angst unabhängig bzw. abhängig von Parität, Varianzanalyse)

\begin{tabular}{l|c|c|c|c|c} 
Effekt & Wilks Lambda & $\mathrm{F}$ & $\mathrm{df}_{1}$ & $\mathrm{df}_{2}$ & Signifikanz \\
\hline Zeit & 0,637 & 12,531 & 3 & 65 & $\mathrm{p}<0,001$ \\
\hline Parität & -- & 0,577 & 1 & 67 & 0,45 \\
\hline Zeit*Parität & 0,936 & 1,493 & 3 & 65 & 0,225 \\
\hline
\end{tabular}

Tabelle 17: Unterschiede zwischen den Messzeitpunkten der State-Angst (Kontrastberechnung)

\begin{tabular}{l|c|c|c} 
Zeitpunkt (Stufe) & df & $F$ & Signifikanz \\
\hline Stufe 1 gegen Stufe 3 & 1 & 38,161 & $\mathrm{p}<0,001$ \\
\hline Stufe 2 gegen Stufe 3 & 1 & 23,397 & $\mathrm{p}<0,001$ \\
\hline Stufe 4 gegen Stufe 3 & 1 & 17,317 & $\mathrm{p}<0,001$ \\
\hline
\end{tabular}

Abbildung 6: State-Angst (Mittelwerte) der verschiedenen Messzeitpunkte

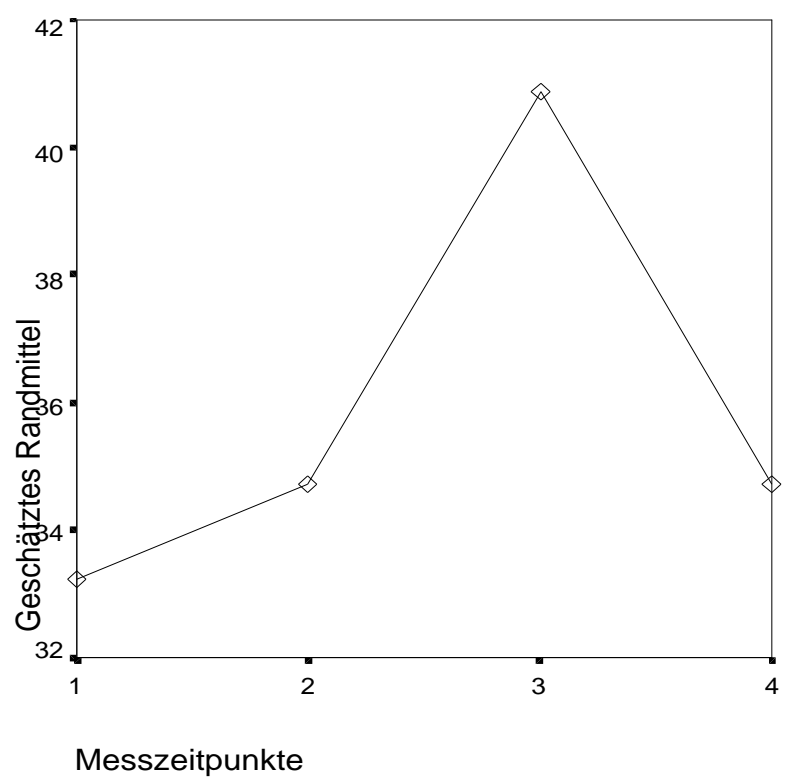




\subsubsection{Trait-Angst (abhängig und unabhängig von Parität)}

Bei der Trait-Angst wurde der Datenlage nach kein signifikanter Mittelwertsunterschied (der beiden Messungen) gefunden. Der Haupteffekt Parität war ebenfalls nicht signifikant: Erstgebärende und Mehrgebärende unterschieden sich nicht signifikant in bezug auf die Trait-Angst (Mittelwerte der zwei Messungen). Jedoch zeigte die durchgeführte zweifaktorielle Varianzanalyse mit Messwiederholung einen signifikanten Interaktionseffekt zwischen Zeit (Messzeitpunkte) und Parität. Demnach ergab sich eine Unterschiedlichkeit des Verlaufs der Trait-Angst. Bei den Erstgebärenden wurde die Angst nach der Geburt grösser, bei den Mehrgebärenden nahm sie ab (Abbildung 7).

Die genauen Ergebnisse der zweikatoriellen Varianzanalyse mit Messwiederholung sind der Tabelle 18 zu entnehmen.

Tabelle 18: Haupteffekte und Interaktion (Trait-Angst abhängig und unabhängig von Parität, Varianzanalyse)

\begin{tabular}{l|c|c|c|c} 
Effekt & $\mathrm{F}$ & $\mathrm{df}_{1}$ & $\mathrm{df}_{2}$ & Signifikanz \\
\hline Zeit & 0,119 & 1 & 69 & 0,731 \\
\hline Parität & 0,766 & 1 & 69 & 0,385 \\
\hline Zeit*Parität & 5,015 & 1 & 69 & 0,028 \\
\hline
\end{tabular}




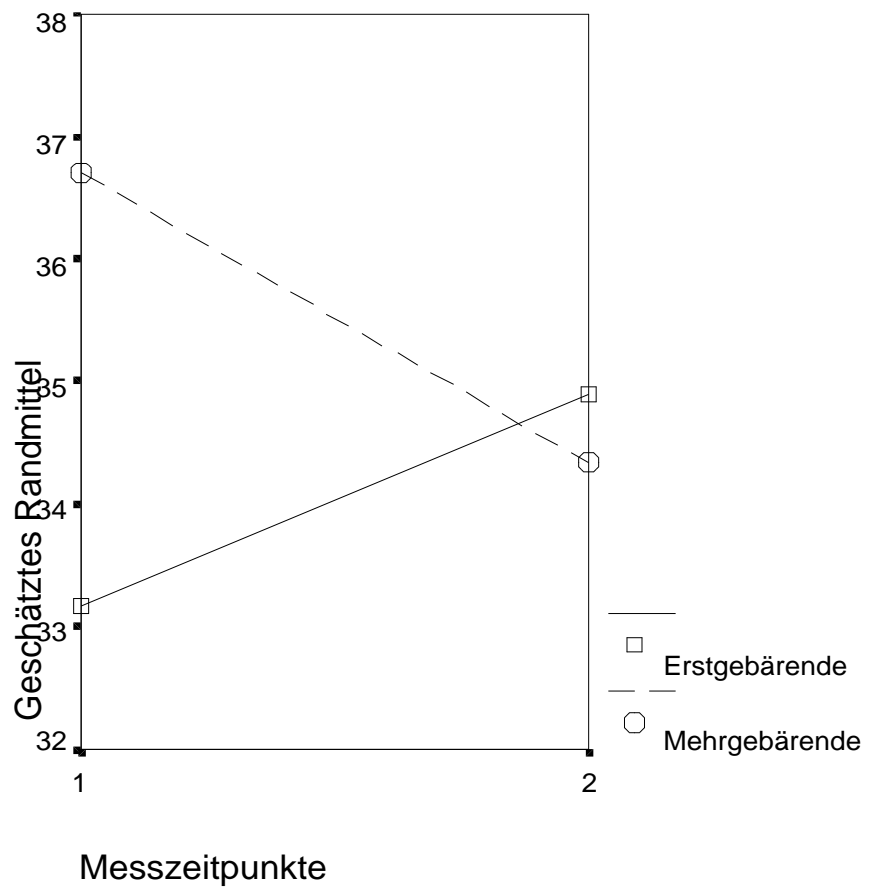

\subsubsection{Interaktionen}

Um Interaktionen zwischen State-Angst (Mittelwert der vier Messzeitpunkte), Bildung, Entbindungsform und Komplikationen festzustellen, wurde eine vierfaktorielle Varianzanalyse mit Messwiederholung durchgeführt. Aufgrund der (teilweise) geringen Anzahl an Fällen pro Bildungsniveau, wurde Bildung in zwei Gruppen zusammengefasst: Hauptschulabschluss und Mittlere Reife (niedriges Niveau) sowie Fachhoch-/Hochschulreife und Fachhoch-/Hochschulabschluss (hohes Niveau). Es ergaben sich weder signifikante Zweifach-, Dreifach- noch Vierfach-Interaktionen, aber einen weiteren signifikanten Haupteffekt. Ausser der Zeit (siehe unter Abschnitt 4.5.1.1) war auch Bildung signifikant. Frauen mit hohem Bildungsniveau hatten generell höhere State-Angstwerte als Frauen mit niedrigem Bildungsniveau. Tabelle 19 sowie Abbildung 8 und 9 zeigen die genauen Ergebnisse.

Tabelle 19: Prüfung auf Interaktionen und Haupteffekte (Varianzanalyse)

\begin{tabular}{l|l|l|l|l|l|l} 
Effekte & Wilks & $\mathrm{F}$ & $\mathrm{df}_{1}$ & $\mathrm{df}_{2}$ & Signifikanz \\
\hline
\end{tabular}




\begin{tabular}{|c|c|c|c|c|c|}
\hline & Lambda & & & & \\
\hline Zeit*Bildung & 0,984 & 0,321 & 3 & 59 & 0,810 \\
\hline ZeitEntbindungsform & 0,898 & 2,237 & 3 & 59 & 0,093 \\
\hline Zeit*Komplikationen & 0,981 & 0,377 & 3 & 59 & 0,770 \\
\hline Zeit*Bildung*Entbindungsform & 0,914 & 1,848 & 3 & 59 & 0,148 \\
\hline Zeit*Bildung*Komplikationen & 0,910 & 1,944 & 3 & 59 & 0,132 \\
\hline ZeitEntbindungsformKomplikationen & 0,985 & 0,309 & 3 & 59 & 0,819 \\
\hline ZeitBildung ${ }^{\star}$ EntbindungsformKomplikationen & 0,976 & 0,489 & 3 & 59 & 0,691 \\
\hline Bildung & -- & 7,267 & 1 & 61 & 0,009 \\
\hline Komplikationen & -- & 0,921 & 1 & 61 & 0,341 \\
\hline Entbindungsform & -- & 2,514 & 1 & 61 & 0,147 \\
\hline
\end{tabular}

Abbildung 8: State-Angst in Abhängigkeit von Bildung, Komplikationen und Klinikgeburt 


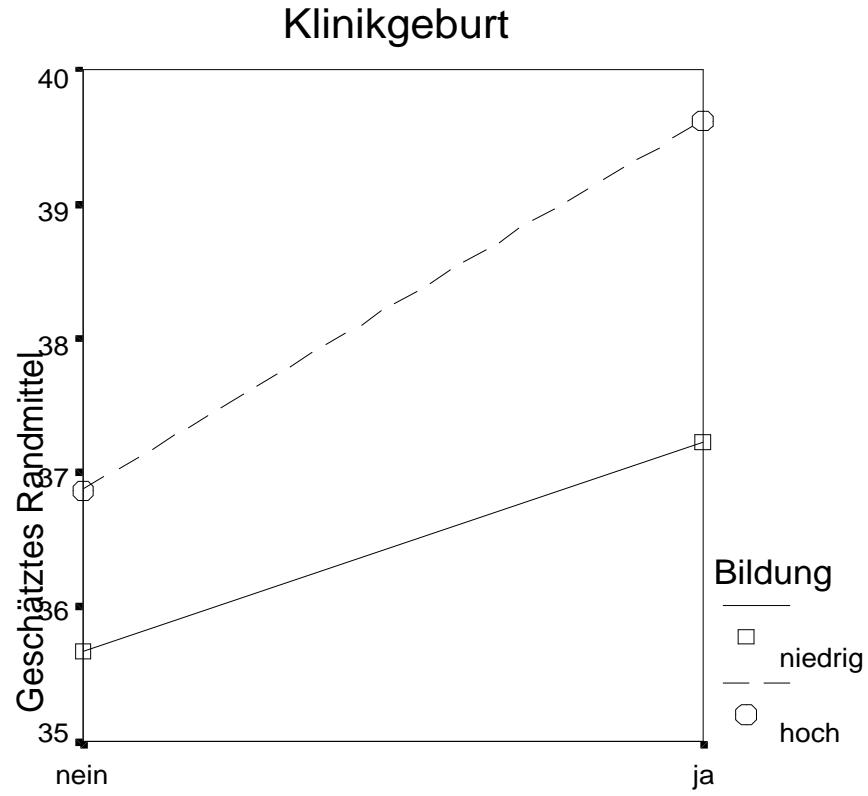

Komplikationen

Abbildung 9: State-Angst in Abhängigkeit von Bildung, Komplikationen und Hausgeburt

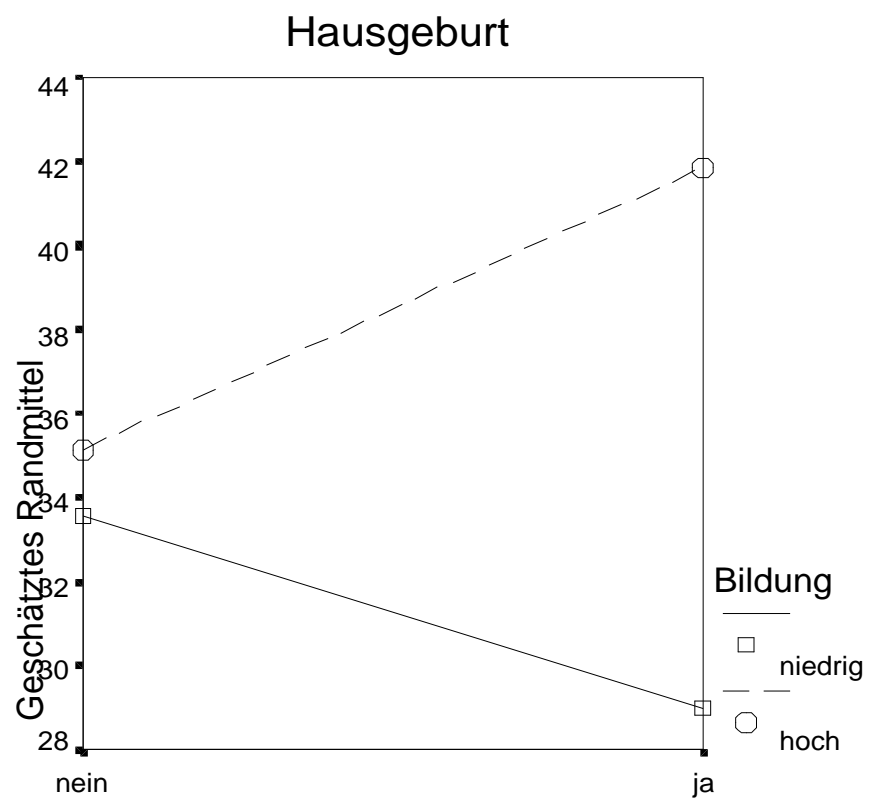

Komplikationen

Um Interaktionen zwischen Trait-Angst (Mittelwert der beiden Messungen), Bildung und Komplikationen zu untersuchen, wurde eine dreifaktorielle Varianzanalyse mit 
Messwiederholung berechnet. Bildung wurde aus oben genannten in niedriges und hohes Niveau kategorisiert (siehe oben). Es stellte sich eine signifikante DreifachInteraktion zwischen Trait-Angst, Bildung und Komplikationen heraus. Es zeigte sich, dass die Trait-Angst nach der Geburt bei allen abnahm, nur bei Frauen mit Komplikationen und niedrigem Bildungsniveau nahm sie zu, wie aus Abbildung 10 und $11 \mathrm{zu}$ sehen ist. Diese Interaktion blieben stabil, auch wenn alle übrigen Zwifach- und Dreifach-Interaktionen und die Vierfach-Interaktion (waren nicht signifikant, siehe Anhang A) berechnet wurden. Darüber hinaus gab es noch einen signifikanten Haupteffekt bezüglich Bildung. Frauen mit höherem Bildungsniveau wiesen global höhere Trait-Angstwerte auf als jene mit niedrigem Bildungsniveau. Die genauen Ergebnisse sind Tabelle 20 zu entnehmen.

Tabelle 20: Prüfung auf Interaktionen und Haupteffekte (Varianzanalyse)

\begin{tabular}{l|c|c|c|c} 
Effekte & $\mathrm{F}$ & $\mathrm{df}_{1}$ & $\mathrm{df}_{2}$ & Signifikanz \\
\hline Zeit*Bildung & 1,605 & 1 & 66 & 0,210 \\
\hline Zeit*${ }^{\star}$ Entbindungsform & 0,449 & 1 & 66 & 0,505 \\
\hline Zeit*Komplikationen & 0,138 & 1 & 66 & 0,712 \\
\hline Zeit*Bildung*Komplikationen & 5,091 & 1 & 66 & 0,027 \\
\hline Bildung & 4,202 & 1 & 66 & 0,044 \\
\hline Komplikationen & 2,092 & 1 & 66 & 0,153 \\
\hline Entbindungsform & 0,538 & 1 & 66 & 0,462 \\
\hline
\end{tabular}




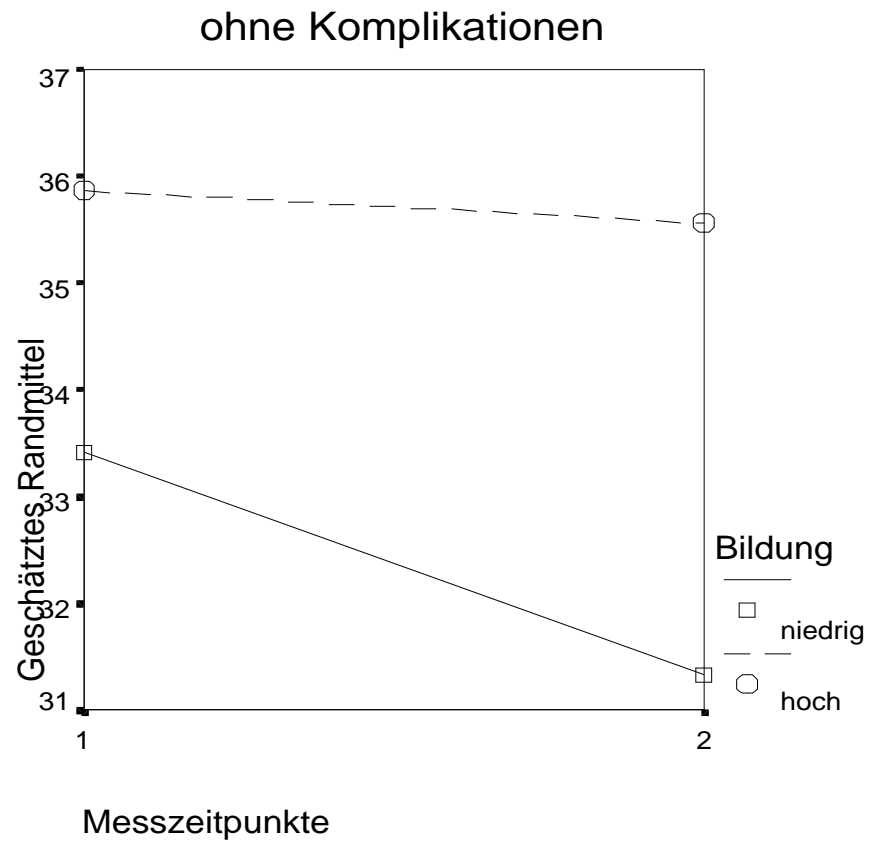

Abbildung 11: Trait-Angst in Abhängigkeit von Bildung und mit Komplikationen

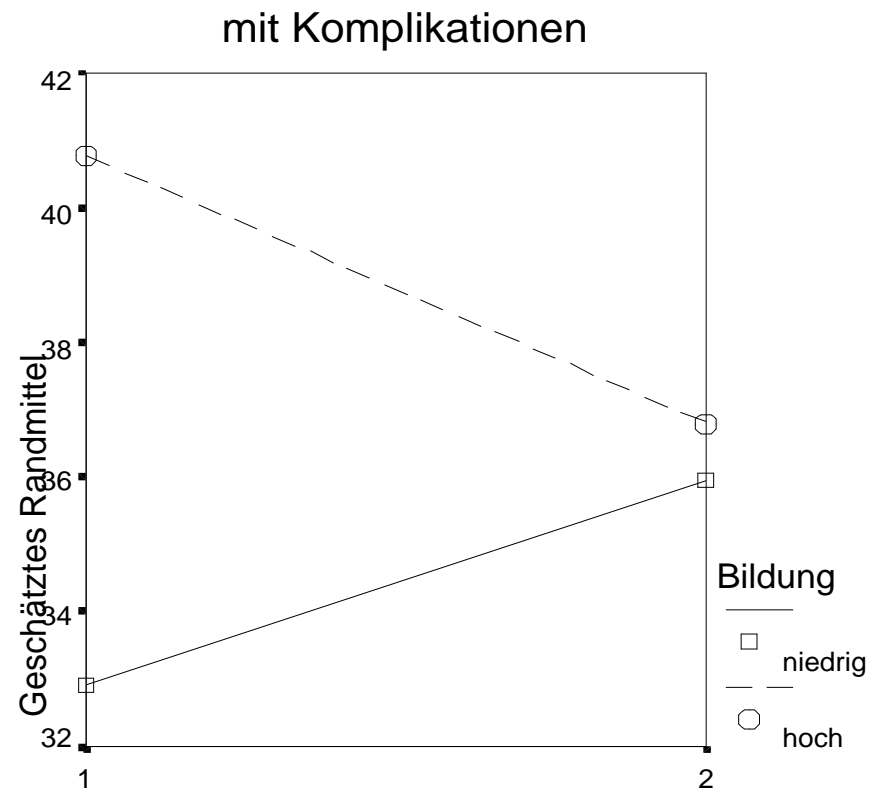

Messzeitpunkte 


\subsubsection{Bestimmung von Prädiktoren}

Um Vorhersagen über Zusammenhänge treffen zu können, wurden binär logistische und lineare Regressionen berechnet.

\section{Vorhersage der Entbindungsform}

Unter Konstanthaltung von den State- und Trait-Werten der ersten Messung, Alter, Fehlgeburt, Parität, Berufstätigkeit und Familienstand eignete sich das Bildungsniveau (Regressionskoeffizienten signifikant positiv: 1,877; $d f=1 ; p<0,05$ ) und der Familienstand (Regressionskoeffizient signifikant positiv:1,902; $\mathrm{df}=1$; $p<0,05)$ als Prädiktor für die Entbindungsform.

Man konnte vorhersagen, dass sich die Wahrscheinlichkeit, für eine Hausgeburt beim Übergang von niedrigem (Hauptschulabschluss und Mittlere Reife) zu hohem Bildungsniveau (Fachhochschul-/Hochschulreife und Fachhochschul- bzw. Hochschulabschluss) erhöhte. Durch die Prädiktorvariable Familienstand konnte vorhergesagt werden, dass sich beim Übergang von Familienstand "nicht ledig“ (entspricht verheiratet und geschieden) zu "ledig“ die Wahrscheinlichkeit für eine Hausgeburt erhöhte. Die in die Regression eingegebenen Variablen erklärten $36,9 \%$ der Varianz (Nagelkerkes $R_{-}=0,369$ Entropiereduktion). Die genauen Ergebnisse sind in Tabelle 21 dargestellt. 
Tabelle 21: Binär logistische Regression zur Vorhersage der Entbindungsform

\begin{tabular}{l|c|c|c|c} 
& Regressionskoeffizient B & Wald $\chi_{-}$ & df & Signifikanz \\
\hline STAI X1,1 & 0,021 & 0,094 & 1 & 0,759 \\
\hline STAI X2,1 & $-0,013$ & 0,044 & 1 & 0,834 \\
\hline STAI X1,2 & $-0,037$ & 1,030 & 1 & 0,310 \\
\hline Alter & 0,007 & 0,006 & 1 & 0,938 \\
\hline Fehlgeburt & 0,939 & 0,878 & 1 & 0,349 \\
\hline Parität & 0,081 & 0,044 & 1 & 0,834 \\
\hline Berufstätigkeit & $-0,832$ & & & \\
\hline $\begin{array}{l}\text { Bildung } \\
\text { (niedrig 0/hoch 1) }\end{array}$ & 1,877 & 6,462 & 1 & 0,011 \\
\hline Familienstand \\
(nicht ledig 0/ledig 1)
\end{tabular}

Vorhersage von Komplikationen

Unter Konstanthaltung von vorgeburtlichen State- und Traitwerten, Alter, Bildung, Entbindungsform und Familienstand, wurde die Parität (Regressionskoeffizient signifikant negativ: $-1,619 / p<0,05)$ als Prädiktor für Komplikationen ermittelt.

Es konnte vorhergesagt werden, dass sich mit zunehmender Parität die Wahrscheinlichkeit für einen Verlauf ohne Komplikationen erhöhte. Es konnte mit den in die Regression eingegebenen Variablen $41,7 \%$ der Varianz aufgeklärt werden (Nagelkerkes $R_{-}=0,417$ Entropiereduktion). Die genauen Ergebnisse sind in Tabelle 22 dargestellt. 
Tabelle 22: Binär logistische Regression zur Vorhersage von Komplikationen

\begin{tabular}{|c|c|c|c|c|}
\hline & Regressionskoeffizient B & Wald Chi_ & df & Signifikanz \\
\hline STAI X1,1 & 0,033 & 0,184 & 1 & 0,668 \\
\hline STAI X2,1 & 0,103 & 1,788 & 1 & 0,181 \\
\hline STAI X1,2 & $<0,001$ & $<0,001$ & 1 & 0,996 \\
\hline STAI X1,3 & 0,014 & 0,118 & 1 & 0,732 \\
\hline Alter & 0,102 & 0,822 & 1 & 0,364 \\
\hline Fehlgeburt & 0,575 & 0,192 & 1 & 0,662 \\
\hline Parität & $-1,689$ & 6,203 & 1 & 0,013 \\
\hline Entbindungsform &,- 083 & 0,012 & 1 & 0,913 \\
\hline Berufstätigkeit & 0,438 & 0,251 & 1 & 0,616 \\
\hline $\begin{array}{l}\text { Bildung } \\
\text { (niedrig 0/hoch 1) }\end{array}$ & $-1,686$ & 3,263 & 1 & 0,071 \\
\hline $\begin{array}{l}\text { Familienstand } \\
\text { (nicht ledig 0/ledig 1) }\end{array}$ & $-1,089$ & 1,073 & 1 & 0,300 \\
\hline Konstante & $-6,840$ & 2,683 & 1 & 0,104 \\
\hline
\end{tabular}

Dass ein Zusammenhang zwischen Parität und Komplikationen besteht, konnte auch durch einen $\chi \_$-Test gezeigt werden. Es bestand ein signifikanter Unterschied in bezug auf Komplikationen und der Parität. Das heisst, dass eine Frau, die noch kein Kind geboren hatte, signifikant häufiger eine Geburtskomplikation hatte, als eine Mehrgebärende. Die genauen Ergebnisse sind Tabelle 23 und Abbildung $12 \mathrm{zu}$ entnehmen. 
Tabelle 23: Parität und Komplikationen ( $\chi_{-}$-Test)

\begin{tabular}{l|c|c} 
Komplikationen & Erstgebärende & Mehrgebärende \\
\hline ohne & $16(33,3 \%)$ & $32(66,7 \%)$ \\
\hline mit & $15(57,7 \%)$ & $11(42,3 \%)$ \\
\hline$\chi_{L}(\mathrm{LR})=4,1 \% ; 0=1$
\end{tabular}

$\chi_{-}(\mathrm{LR})=4,1^{*} ; \mathrm{df}=1$

${ }^{*} p<0,05$

Abbildung 12: Parität und Komplikationen ( $\chi_{-}-$Test)

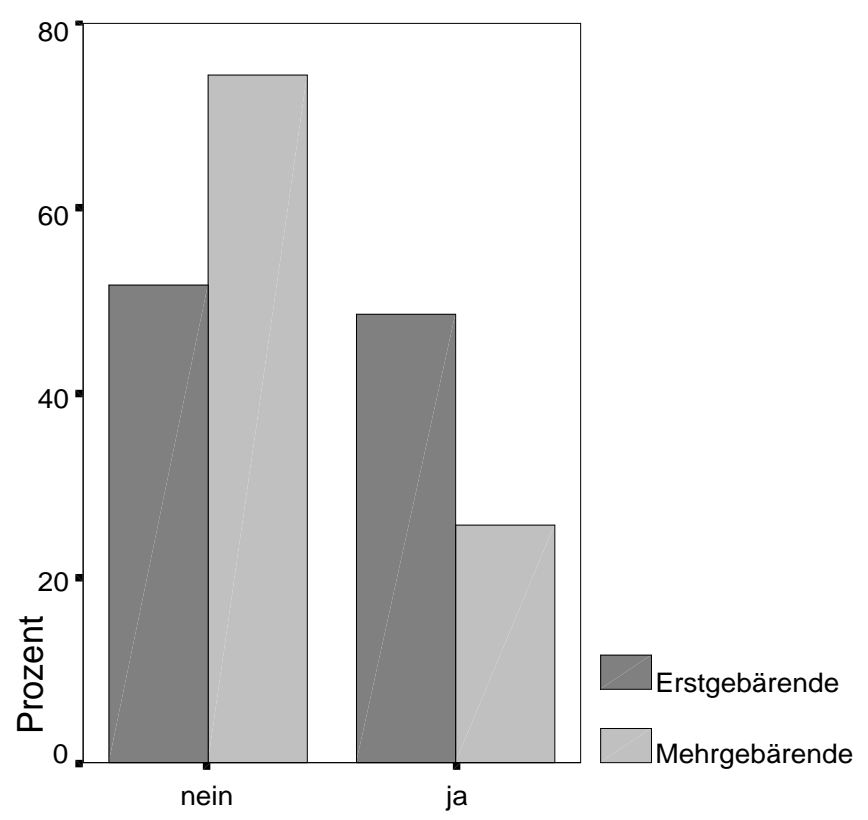

Komplikationen

Vorhersage der Angst bei Geburtsbeginn(STAI X1,3)

Unter Konstanthaltung von STAI X1,1, Alter, Fehlgeburt, Parität, Komplikationen, Berufstätigkeit und Bildung ermittelte die lineare Regression den STAIX2,1 und die Entbindungsform als Prädiktorvariablen.

Mit dem STAI X2,1 liess sich der STAI X1,3 vorhersagen (Koeffizient B signifikant positiv: 0,504<0,05). Durch die Entbindungsform wurde die Vorhersage möglich, 
dass sich beim Übergang von Klinikgeburt zu Hausgeburt, der STAI X1,3 um 6 Punkte reduzierte (Koeffizient $B$ signifikant negativ: $-6,147 ; p<0,05$ ).

Ursprünglich ging in dieses Modell auch der STAI X1,2 mit ein. Jedoch musste er wegen Kolinearitätsproblemen herausgenommen werden (VIF $>3$ ). Die in der Regression verbliebenen Variablen konnten $23,6 \%$ der Varianz erklären (korrigiertes signifikantes $R_{-}=0,236$ Entropiereduktion, $F(9 / 57)=3,271 ; p<0,05$ ). Die genauen Ergebnisse sind Tabelle 24 zu entnehmen.

Tabelle 24: Lineare Regression zur Vorhersage von STAI X 1,3

\begin{tabular}{|c|c|c|c|}
\hline & $\begin{array}{c}\text { Nicht standardisierte } \\
\text { Koeffizienten B }\end{array}$ & $\mathrm{T}$ & Signifikanz \\
\hline STAI X1,1 & 0,117 & 0,417 & 0,678 \\
\hline STAI X2,1 & 0,504 & 2,075 & 0,043 \\
\hline Alter & $-0,195$ & $-0,523$ & 0,603 \\
\hline Fehlgeburt & 1,237 & 0,339 & 0,736 \\
\hline Parität & 1,206 & 0,733 & 0,467 \\
\hline Beruftätigkeit & 0,345 & 0,110 & 0,913 \\
\hline $\begin{array}{l}\text { Bildung } \\
\text { (niedrig 0/hoch 1) }\end{array}$ & 0,544 & 0,179 & 0,671 \\
\hline $\begin{array}{l}\text { Familienstand } \\
\text { (nicht ledig 0/ledig 1) }\end{array}$ & $-4,628$ & $-1,359$ & 0,179 \\
\hline $\begin{array}{l}\text { Entbindungsform } \\
\text { (Klinik 0/Haus 1) }\end{array}$ & $-6,147$ & $-2,325$ & 0,024 \\
\hline Konstante & 28,147 & 2,267 & 0,027 \\
\hline
\end{tabular}




\subsection{Zusammenfassung der Ergebnisse}

\subsubsection{Hypothesen}

Da die Stichprobe nur eine Frau mit Schwangerschaftskomplikation enthielt, konnte der Zusammenhang zwischen Angst und Schwangerschaftskomplikationen (Hypothese 1 a) nicht überprüft werden.

Hypothese $1 \mathrm{~b}$ wurde von der Datenlage nicht bestätigt. Es bestand folglich kein Zusammenhang zwischen Geburtskomplikationen und Angst. Allerdings fand sich ein Zusammenhang zwischen Komplikationen der Kategorie 3 (sonstige Komplikationen) und Angst. Diese Frauen hatten signifikant höhere StateAngstwerte.

Hypothese $2 \mathrm{a}(\mathrm{H}$ 0) postulierte, dass sich die Komplikationsrate von Hausgeburten und Klinikgeburten nicht unterscheidet. Diese Hypothese bewährte sich der Datenlage nach. Die Hypothese 2 b ( H 1, Alternativhypothese) musste somit verworfen werden. Jedoch konnte ein signifikanter Zusammenhang zwischen Komplikationen in der Nachgeburtsperiode sowie Mehrfachkomplikationen und Entbindungsform festgestellt werden. Diese Komplikationen traten signifikant häufiger in der Klinikgeburtsgruppe auf.

Hypothese 3, welche Unterschiede zwischen Angst und Entbindungsform untersuchte, wurde von der Datenlage nicht bestätigt. Demnach unterschieden sich Hausgeburts- und Klinikgeburtsfrauen nicht bezüglich Angst (State- und TraitAngst).

\subsubsection{Explorative Analysen}

\subsubsection{State- und Trait-Angst (unabhängig von der Entbindungsform)}

Zwischen dem dritten Messzeitpunkt der State-Angst und allen drei anderen Messzeitpunkten bestanden signifikante Unterschiede. Bei der Trait-Angst ergab sich kein signifikanter Unterschied. Es zeigte sich aber eine signifikante Interaktion zwischen Zeit und Parität. 


\subsubsection{Interaktionen}

Es wurde eine signifikante Dreifach-Interaktion zwischen Trait-Angst, Bildung und Komplikationen festgestellt. Ausserdem gab es signifikante Haupteffekte bezüglich Bildung. Sowohl die State-Angst als auch die Trait-Angst war bei Frauen mit höherer Bildung grösser.

\subsubsection{Bestimmung von Prädiktoren}

Unter Konstanthaltung der anderen in das Regressionsmodell eingegebenen Variablen, konnten Prädiktorvariablen für die Entbindungsform, Komplikationen und dem STAI X1,3 ermittelt werden. Die Vorhersage der Entbindungsform war durch Bildung und Familienstand möglich. Komplikationen konnten mit der Parität vorhergesagt werden. Die Vorhersage des STAI X 1,3 war durch den STAI X2,1 und die Entbindungsform möglich. 


\section{Diskussion}

Aus der Verhaltensmedizin ist der Einfluss von Angst schon seit vielen Jahren sehr gut bekannt. Angst führt unter anderem zu muskulärer Anspannung und kann zur Entstehung diverser Krankheiten beitragen (z.B. kardiovaskuläre Erkrankungen, Migräne, usw.; Melamed \& Siegel, 1980; Sartory \& Diener, 2001). Im psychosomatischen Bereich ist der Einfluss von Angst auf Schmerzen sehr gut erforscht (Flor \& Hermann, 1999). Jedoch wurde dem Einfluss von Angst auf die Geburt bisher eher wenig Beachtung geschenkt.

Die Geburt stellt einen psychobiologischen Vorgang und eine psychosoziale Situation dar. Noch bis vor 50-60 Jahren erblickten die meisten Kinder zu Hause im Kreise der Familie das Licht der Welt. Gründe für die Hausgeburt waren deutlich niedrigere Kosten, praktische Gründe wie die Nähe zur Familie (Versorgung der anderen Kinder, Haushalt usw.), und oftmals wäre der Transport in die nächstgelegene Klinik mit den damaligen Möglichkeiten nur schwer zu bewerkstelligen gewesen. Die Geburt wurde als ein natürlicher Vorgang betrachtet und erfolgte zu Hause. Die Klinikgeburt stellte eine Ausnahme dar (DaviesOsterkamp \& Beckmann, 1982).

Mit dem Fortschreiten der Wissenschaft und Technik und den verbesserten Sozialleistungen hat sich dieses Bild deutlich gewandelt (European Workgroup of Independent Midwives, 2000). In Deutschland kommen heute ca. 99\% aller Kinder in Kliniken auf die Welt. Obwohl man in anderen Ländern wie z.B. Holland die Vorteile der Hausgeburt immer noch zu schätzen weiss (Wiegers et al., 1996), die dortige Hausgeburtsrate beträgt ca. 35\% (Treffers et al., 1986), ist sie in Deutschland eine kaum wahrgenommene Alternative. 
Die vorliegende Diplomarbeit hat sich der Untersuchung von Angst im Zusammenhang von Schwangerschaft und Geburt sowie dem Aspekt der Entbindungsform zugewandt.

In diesem Kapitel werden zunächst die wichtigsten Ergebnisse zusammengefasst und mit empirischen Forschungsergebnissen diskutiert. Anschliessend wird auf die Stärken und Schwächen der vorliegenden Untersuchung eingegangen und ein Ausblick für zukünftige Forschung gegeben.

\subsection{Diskussion der Ergebnisse}

\subsubsection{Stichprobe}

Die soziodemographischen Daten zeigten signifikante Unterschiede zwischen den Gruppen. Hausgeburtsfrauen hatten ein signifikant höheres Bildungsniveau. In der Literatur sind vergleichbare Befunde kaum zu finden, da fast alle Stichproben nach dem "Matching-Prinzip" gewonnen wurden. Aus Holland gibt es eine vergleichbare Studie. Kleiverda, Steen, Andersen, Treffers \& Everaerd (1990) kamen in ihrer prospektiven Längsschnittstudie zu dem Ergebnis, dass Frauen, die eine Hausgeburt planten, ein hochsignifikant höheres Bildungsniveau aufwiesen als Frauen, die eine Klinikgeburt planten.

Im Folgenden werden denkbare Erklärungen präsentiert, die für ein höheres Bildungsniveau bei Hausgeburtsfrauen sprechen. Eine Frau, die über einen höheren Bildungsstand verfügt, ist wahrscheinlich zu einer objektiveren, kritischeren Auseinandersetzung mit strittigen Themen fähig. Sie lässt sich nicht so leicht von einem Vorhaben abbringen, auch wenn es von anderen teilweise kritisch beurteilt wird. Sie setzt bewusst ihre Fähigkeiten ein, sich aussagekräftige Informationen einzuholen (z.B. Internetrecherche) und bildet sich eine eigene Meinung. Eine gebildete Frau kann aufgrund ihrer Informationslage und gesunden Menschenverstands, Risiken möglicherweise besser und selbständiger einschätzen. Eventuell ist eine solche Frau auch eher bereit, mehr Selbstverantwortung für sich und das Kind zu übernehmen. Solche Frauen entscheiden sich vermutlich häufiger für eine Hausgeburt. 
Auf Nachfrage bei Hebammen, die seit Jahren in der Hausgeburtshilfe tätig sind, wurde der höhere Bildungsstand bestätigt.

Ein weiterer signifikanter Unterschied bestand in bezug auf den Familienstand. Unter den Hausgeburtsfrauen gab es signifikant mehr ledige Frauen als unter den Klinikgeburtsfrauen.

Befunde aus der Literatur sind sehr spärlich. Gründe dafür sind unter anderem, dass die meisten Stichproben mit dem „Matching-Verfahren“ gebildet wurden (siehe oben). Kleiverda et al. (1989) konnten keinen Zusammenhang zwischen Familienstand und Entbindungsform feststellen.

Denkbare Gründe für die Ergebnisse vorliegender Untersuchung werden im Folgenden dargestellt. Möglicherweise sind Frauen, die sich für eine Hausgeburt entscheiden, weniger konservativ. Die Hausgeburt stellt schliesslich eine Aussnahme dar - für gewöhnlich gebären Frauen in einer Klinik. So könnte man weiter vermuten, dass diese weniger konservative Einstellung die Wahrscheinlichkeit erhöht, trotz Kind(ern) nicht zu heiraten. In Deutschland überwiegt der Anteil an verheirateten Eltern, weshalb man daraus schliessen kann, dass es üblich ist, zu heiraten. Eine Studie des Bundesministers für Familie, Senioren, Frauen und Jugend (1998) zeigte, dass in den alten Bundesländer 1995 89\% aller unter Dreijährigen in Ehepaar-Haushalten lebten (alle minderjährigen Kinder zu 85\%). Kleiverda et al. (1989) zeigten in ihrer Studie, dass eine weniger konservative Einstellung gegenüber der weiblichen sozialen Rolle ein Prädiktor für eine Hausgeburt war. Dieser Befund spricht dafür, dass Hausgeburtsfrauen weniger konservativ sind.

Wünschenswert ist für die weitere Klärung, dass man bei der Erfassung der ausserklinischen Geburten in Deutschland bzw. bei zukünftigen Untersuchungen, das Bildungsniveau und den Familienstand mit erhebt (sofern kein "Matching“ erfolgt). 


\subsubsection{Angst und Komplikationen}

Die Hypothese 1 b, welche den Zusammenhang zwischen Angst und Komplikationen untersuchte, wurde von der Datenlage nicht bestätigt. Frauen mit und ohne Geburtskomplikationen unterschieden sich bezüglich State- und Trait-Angst nicht.

Es sind in der Literatur vergleichbare Befunde zu finden, wie z.B. die Untersuchung von Molfese et al. (1987). Andererseits gibt es empirische Beweise für einen Zusammenhang. Rizzardo et al. (1988) fanden bei Frauen mit Geburtskomplikationen signifikant höhere State-Angstwerte als bei Frauen ohne Geburtskomplikationen. Allerdings bestand der signifikante Unterschied nur im dritten Schwangerschaftsmonat. Bei der Erhebung im sechsten und neunten Schwangerschaftsmonat sowie nach der Geburt gab es keine signifikanten Gruppenunterschiede. Diese Befunde stimmen auch mit den Ergebnissen von Gorsuch \& Key (1974) überein, die nur für den dritten, vierten und sechsten Schwangerschaftsmonat signifikante Unterschiede bestätigen konnten (bis zum Zeitpunkt der Geburt keine signifikanten Unterschiede mehr vorhanden).

Bezüglich der Trait-Angst gibt es übereinstimmende Befunde. Rizzardo et al. (1988) konnten keine Unterschiede zwischen Frauen mit und ohne Komplikationen finden. Allerdings ist nur das Ergebnis der postpartalen Erhebung ohne Einschränkung vergleichbar. Der präpartale Erhebungszeitpunkt ist bei Rizzardo et al. (1988) erheblich früher (dritter Schwangerschaftsmonat) als in vorliegender Diplomarbeit, was einen Vergleich nur bedingt möglich macht.

Insofern stehen die Ergebnisse der vorliegenden Diplomarbeit im Einklang mit der bestehenden Literatur bzw. widersprechen ihr nicht.

Die Hypothese $1 \mathrm{~b}$ basiert auch auf meinen Erfahrungen als Hebamme und denen von Kolleginnen mit langjähriger Berufserfahrung. Der Eindruck, das ängstlichere Frauen prädisponiert für Komplikationen sind, konnte empirisch nicht bestätigt werden. Möglich ist, dass Frauen mit Komplikationen von Hebammen anders 
wahrgenommen werden, als sie sich selbst einschätzen. Hebammen können bei diesen Frauen sogar das entsprechende Verhalten provozieren. Hier ist ein Erwartungseffekt denkbar (Huber, 1995). Hebammen könnten vermuten, dass eine Frau mit Geburtskomplikation, auch ängstlicher ist.

Als Gründe für die nicht signifikanten Ergebnisse kommen unter anderem folgende in Frage:

Wie aus der Literatur bekannt ist, gibt es weitere Variablen, die mit Geburtskomplikationen in Zusammenhang stehen. So wird zum Beispiel von McDonald \& Christakos (1963), McDonald \& Parham (1964) und Windsor-Oettel (1992) ein niedriges Selbstwertgefühl mit Komplikationen in Verbindung gebracht. Lukesch und Lukesch (1976) stellten fest, dass Frauen mit verzögerten Geburtverläufen aufgrund starker Verkrampfungen eine erhöhte Ablehnung der Schwangerschaft und eine negativere Haltung gegenüber der Sexualität aufwiesen. Die Ergebnisse von Molfese et al. (1987) und Rauchfuss (1996) unterstreichen die synergetische Wirkung von Variablen wie Angst, Schwangerschaftseinstellungen, "Locus of Control“, Partnerschaft, Stress und wahrgenommene soziale Unterstützung.

Betrachtet man den Zusammenhang von Angst und Komplikationskategorien, stellt sich eine signifikante Verbindung zwischen State-Angst und der Kategorie 3 "Sonstige Komplikationen“ heraus. In der vorhandenen Literatur findet man wenige derartigen Untersuchungen, wobei dies für die zukünftige Forschung ein interessanter Ansatzpunkt wäre. So könnte gezeigt werden, dass spezifische Komplikationen mit Angst zusammenhängen. Perkin et al. (1993) analysierten den Zusammenhang von speziellen Komplikationen und Angst. Sie konnten aber keine Beziehung feststellen. Beck et al. (1980) und Falorni et al. (1979) stellten eine Beziehung zwischen protrahiertem Geburtsverlauf und hoher Ängstlichkeit fest. Dieses Ergebnis konnte in vorliegender Arbeit nicht bestätigt werden. Der protrahierte Geburtsverlauf fällt in Komplikationskategorie 1, für die kein signifikanter Zusammenhang mit State- sowie Trait-Angst festgestellt wurde. 
Für weitere Untersuchungen ist es sinnvoll, Angst schon ab dem ersten Trimenon der Schwangerschaft zu untersuchen und oben genannte weitere Einflussfaktoren mit in die Untersuchung einzubeziehen.

\subsubsection{Komplikationen und Entbindungsform}

Die Hypothese $2(\mathrm{H} 0)$ postulierte, dass sich geplante Hausgeburten und geplante Klinikgeburten bezüglich der Komplikationsrate nicht unterscheiden. Aufgrund der Datenlage bewährte sich diese Hypothese. Ein Zusammenhang zwischen Angst und geplanter Haus- bzw. Klinikgeburt konnte nicht gezeigt werden.

Dieser Befund deckt sich mit aus der Literatur bekannten Befunden. Die Weltgesundheitsorganisation (WHO, 1987, zit. nach Zimmermann, 1998) nimmt in einer offiziellen Verlautbarung zum Thema Geburtshilfe Stellung und postuliert, dass es keinen wissenschaftlichen Anhaltspunkt gibt, der belegt, dass eine geplante Hausgeburt für eine Frau, die einen normalen Schwangerschaftsverlauf hatte, mehr Gefahren mit sich bringt als eine Geburt in der Klinik. Viele internationale Studien konnten diese These belegen. Studien aus z.B. der Schweiz (Ackermann-Liebrich et al., 1997) und England (Macfarlane et al., 2000) liefern empirische Beweise für die Gleichheit bezüglich der Sicherheit beider Entbindungsformen.

Für Deutschland liegen nur wenige empirische Befunde vor. Ein möglicher Grund dafür könnte die schlechte Lobby und das Desinteresse an Hausgeburten sein. Die meisten Ärzte in Deutschland lehnen die Hausgeburtshilfe inklusive weiterer Formen der ausserklinischen Geburtshilfe strikt ab. So auch die Deutsche Gesellschaft für Gynäkologie und Geburtshilfe (Linder, 1994). Aus eigener Erfahrung als Hebamme kann ich diese Haltung bestätigen. Es gibt Ärzte (und auch Hebammen), die Frauen, die an einer Hausgeburt interessiert sind, mit der Aussage „Wollen Sie Ihr Kind umbringen?" konfrontieren. Diese Unterstellung ist jedoch auf wissenschaftlicher Grundlage nicht tragbar.

Diesem Missstand entgegenzuwirken hat sich die Gesellschaft für Qualität in der ausserklinischen Geburtshilfe e.V. zur Aufgabe gemacht. Wie im klinischen Bereich 
findet seit 1999 auch eine Erfassung der Geburten im ausserklinischen Bereich statt (initiiert durch die Gesellschaft für Qualität in der ausserklinischen Geburtshilfe e.V.). Der Erfassungsgrad liegt mittlerweile bei $72 \%$. Die bisherigen Qualitätsberichte (1999, 2000 und 2001) oben genannter Gesellschaft stehen im Einklang mit den internationalen Befunden. In diesem Sinne können die diesbezüglichen Ergebnisse der vorliegenden Diplomarbeit als Unterstützung deutscher empirischer Befunde angesehen werden.

Die Alternativhypothese $\left(\begin{array}{lll}H & 1\end{array}\right)$ wurde aufgrund des vorliegenden Datenmaterials verworfen. Demzufolge ging eine geplante Hausgeburt nicht mit weniger Komplikationen einher als eine geplante Klinikgeburt.

Literaturbefunde, die nahe legen, dass Hausgeburten sicherer sind (z.B. Kraker zit. nach Zimmermann, 1998), konnten mit vorliegenden Daten nicht unterstützt werden. Anzumerken ist, dass lediglich wenige Forschungsarbeiten zu dieser Fragestellung existieren. In diesem Zusammenhang wäre es interessant zu wissen, ob die Untersuchungen, die belegen, dass geplante Hausgeburten kein höheres Risiko für Mutter und Kind darstellen, ihre Ergebnisse auf zweiseitige Signifikanz oder nur einseitig geprüft haben, was in den Studien nicht transparent ist. In diesen Untersuchungen geht es immer darum, die Gleichheit beider Entbindungsformen zu zeigen. Anzunehmen ist aber (aus rein methodischen Gründen), dass die Signifikanzprüfung zweiseitig erfolgte. Damit würden die überwiegenden empirischen Evidenzen dafür sprechen, dass die geplante Hausgeburt nicht sicherer ist als die Klinikgeburt, was dem Ergebnis vorliegender Untersuchung entspricht.

Die Hypothese $2(\mathrm{H} 1)$ basiert auch auf meinen Erfahrungen als Hebamme, wobei meine Erfahrungen in der ausserklinischen Geburtshilfe sehr viel geringer sind als die klinischen Erfahrungen. Aufgrund dieser Erfahrungsdiskrepanz habe ich einen Vergleich zweier Gruppen angestellt, obwohl die Gruppe „ausserklinische Geburten" möglicherweise nicht repräsentativ war. Zudem fehlte mir eventuell die nötige Objektivität für den Vergleich. Da ich die ausserklinische Geburtshilfe 
befürworte, insbesondere die Hausgeburtshilfe, wäre es denkbar, dass ich Geburten mit Komplikationen in der Klinik überbewertet habe. Möglicherweise habe ich ausser Acht gelassen, dass Frauen mit Geburtskomplikationen teilweise nicht für eine Hausgeburt in Frage gekommen wären, da sie mit Risikofaktoren belastet waren, die als Kontraindikation für eine Hausgeburt gegolten hätten.

Die zusätzlichen Analysen des Zusammenhangs von Komplikationskategorien sowie Mehrfachkomplikationen und Entbindungsform erbrachten signifikante Gruppenunterschiede. Komplikationen der Kategorie 2, Komplikationen in der Nachgeburtsperiode, und Mehrfachkomplikationen (mehr als eine Komplikation), traten signifikant häufiger in der Gruppe der Klinikgeburtsfrauen auf.

Ein vergleichbarer Befund konnte bei Wiegers et al. (1996) gefunden werden. Sie konnten zeigen, dass Komplikationen in der Nachgeburtsperiode signifikant häufiger in der Klinik auftraten. Allerdings galt dies nur für Mehrgebärende. Zwischen erstgebärenden Hausgeburtsfrauen und Klinikgeburtsfrauen konnte dieser Unterschied nicht gezeigt werden. Vorliegende Studie konnte diesen Zusammenhang aber sowohl für Erstgebärende als auch Mehrgebärende zeigen (Analysen siehe in Anhang A). Nach Wiegers et al. (1996) wiesen mehrgebärende Klinikgeburtsfrauen auch eine signifikant höhere Rate an protrahierten Geburtsverläufen auf. In der vorliegenden Diplomarbeit konnte weder für Erstgebärende noch für Mehrgebärende ein derartiger Zusammenhang festgestellt werden (der protrahierte Geburtsverlauf zählt zur Komplikationskategorie 1, Analysen siehe in Anhang A).

Für weitere Forschungsarbeiten sind dies interessante Zusatzfragestellungen. Bestehende Literatur könnte man dahingehend metaanalysisch untersuchen.

\subsubsection{Angst und Entbindungsform}

Die Hypothese 3, welche Unterschiede zwischen Hausgeburts- und Klinikgeburtsfrauen bezüglich Angst untersuchte, wurde von der Datenlage nicht bestätigt. Es konnte nicht gezeigt werden, dass Frauen, die eine Hausgeburt planten, während der Schwangerschaft, vor und nach der Geburt, ein niedrigeres Angstniveau aufwiesen als jene, die eine Klinikgeburt planten. 
In der Literatur sind vergleichbare Befunde zu finden. Die Untersuchung von Windsor-Oettel (1992) ergab ebenfalls einen nichtsignifikanten Unterschied, der allerdings nur sehr knapp nicht signifikant war $(p=0,0558$; in der vorliegenden Diplomarbeit $p=0,061)$. Diese Ergebnisse rufen nach weiteren empirischen Untersuchungen. Sinnvoll sind Studien, die Angst auch schon mit Beginn der Schwangerschaft erfassen. Denkbar ist nämlich, dass die Unterschiede bezüglich Angst in einem früheren Stadium der Schwangerschaft grösser sind. Dies würde in die Richtung der Untersuchungen gehen, die sich mit dem Zusammenhang von Angst und dem Auftreten von Komplikationen befassen und nur in den ersten sechs Monaten der Schwangerschaft einen Zusammenhang zwischen Komplikationen und Angst zeigen konnten (Gorsuch \& Key, 1974; Rizzardo et al. 1988). Eine weitere sinnvolle Variante ist die spezifische Erfassung von Geburtsangst, mit einem dafür geeigneten Messinstrument wie die Geburts-AngstSkala von Lukesch (1983). Ob sich die Ergebnisse dann ändern, ist eine spannende Fragestellung.

Hypothese 3 entstand aufgrund meines beruflichen Hintergrunds als Hebamme. Meine langjährigen Erfahrungen und die vieler Kolleginnen, legen die Vermutung nahe, dass sich Hausgeburtsfrauen in bezug auf Angst von Klinikgeburtsfrauen unterscheiden. Möglich wäre, dass Hausgeburtsfrauen vom Hebammen anders wahrgenommen werden, als sie sich selbst einschätzen oder, dass Hebammen sogar ein derartiges Verhalten hervorrufen. Hier kann ein Erwartungseffekt eine Rolle spielen (Huber, 1995). Möglicherweise haben Hebammen die Vermutung, dass eine Hausgeburtsfrau auch weniger ängstlicher ist.

\subsubsection{Weitere durchgeführte Untersuchungen}

5.1.5.1 State- und Trait-Angst unabhängig von der Gruppenzugehörigkeit nach Entbindungsform (abhängig und unabhängig von der Parität)

Die isolierte Analyse der State-Angst zeigte ein signifikantes Ergebnis. Signifikante Veränderungen der State-Angst konnten zwischen allen Messzeitpunkten zum dritten Messzeitpunkt „Geburtsbeginn“ gezeigt werden. 
Dass die Angst mit Beginn der Geburt erheblich steigt, ist auch aus der Literatur bekannt. Ashbury (1980) stellte in ihrer Untersuchung fest, dass State-Angst das höchste Niveau mit Wehenbeginn erreicht. Allerdings ist die untersuchte Stichprobe nicht repräsentativ, weshalb ein Vergleich nur mit Vorsicht angestellt werden kann. Nur sehr junge Erstgebärende wurden untersucht.

Der hohe Angstwert bei Geburtsbeginn lässt sich gut mit dem Angst-SpannungsSchmerz-Kreislauf, basierend auf den Beobachtungen von Dick-Read (Lukesch, 1981; wie im Theorieteil ausgeführt), erklären. Nach diesem Modell lösen Wehen Angst aus, die zu Spannung führt, diese löst wiederum Schmerz aus und verstärkt die Angst, usw. Da zu den anderen Messzeitpunkten kein Wehenschmerz besteht, ist nachvollziehbar, dass beim Geburtsbeginn mit Wehen die Angst höher ist.

Erhöhte Angst bei Geburtsbeginn deckt sich auch mit meinen eigenen Erfahrungen als Hebamme. Frauen haben in dieser Phase häufig kalt-schweissige Hände, teilweise einhergehend mit erhöhter Pulsfrequenz und erhöhtem Blutdruck.

Für den signifikanten Unterschied zwischen der Messung bei Geburtsbeginn und postpartal gibt es eingeschränkt vergleichbare Literatur.

Ashbury (1980) stellte fest, dass sich die unter der Geburt gemessene State-Angst signifikant von präpartaler (im dritten Trimenon) und postpartaler Messung unterscheidet (methodische Probleme siehe oben). Windsor-Oettel (1992) kam zu dem gleichen signifikanten Ergebnis, wobei die Messung vor der Geburt, zeitlich nicht konkret festgelegt wurde. Die Messung erfolgte mit der G-A-S. Da die Messung bei Geburtsbeginn in der vorliegenden Diplomarbeit die Absicht hatte, Geburtsangst zu erfassen, scheint das unterschiedliche Messinstrument weniger ins Gewicht zu fallen. Trotzdem bleiben die Untersuchungen nur bedingt vergleichbar. Dass zwischen den anderen Zeitpunkten keine signifikanten Unterschiede bestehen, ist mit der vorhandenen Literatur nur eingeschränkt übereinstimmend. Für die Stabilität zwischen der ersten und zweiten Messung, liegen von Rizzardo et al. (1988) vergleichbare Ergebnisse vor. Hingegen konnten oben genannte Forscher die Stabilität von State-Angst zwischen sechstem bzw. neunten Schwangerschaftsmonat (entspricht der ersten bzw. zweiten Messung 
vorliegender Untersuchung) und der postnatalen Messung, nur bei Frauen mit komplikationslosen Geburten feststellen.

$\mathrm{Zu}$ entgegengesetzten Befunden kam Ashbury (1980), die einen signifikanten Unterschied auch zwischen präpartaler (im dritten Trimenon) und postpartaler State-Angst bei der Untersuchung an ausschliesslich sehr jungen Erstgebärenden zeigen konnte. Aussagekräftiger sind die Ergebnisse von Lunenfeld, Rosenthal, Larholt \& Insler (1984). Bei der Untersuchung einer repräsentativen Stichprobe wurden signifikante Unterschiede zwischen präpartaler und postpartaler bei der State-Angst gefunden. Die Messung vor der Geburt entspricht der ersten in vorliegender Diplomarbeit, die postpartale Messung erfolgte aber innerhalb der ersten 48 Stunden nach der Geburt. Somit sind die Untersuchungen nicht ohne Vorbehalt zu vergleichen.

In bezug auf die Trait-Angst konnte der Datenlage nach kein signifikanter Unterschied gefunden werden. Somit blieb die Trait-Angst zwischen dem prä- und postpartalen Erhebungszeitpunkt stabil, unabhängig von der Gruppenzugehörigkeit. Jedoch ist $z u$ beachten, dass sich eine signifikante Interaktion zwischen Parität und Zeit herausstellte. Bei den Erstgebärenden nahm die Trait-Angst nach der Geburt zu, bei den Mehrgebärenden wurde sie geringer. Deshalb kann man nur bedingt daraus schliessen, dass die Geburt eines Kindes keinen Einfluss auf Angst als Eigenschaft hat. Bei dieser Frage ist es wichtig zu wissen, ob es sich um eine Erst- oder Mehrgebärende handelt.

Die Stabilität der Trait-Angst wurde auch in anderen Studien gefunden. Rizzardo et al. (1988) konnten in ihrer Untersuchung die Stabilität der Trait-Angst zeigen. Allerdings wird in dieser Studie die Variation zwischen drittem Schwangerschaftsmonat (in der vorliegenden Diplomarbeit ca. siebter Schwangerschaftsmonat) und nach der Geburt untersucht.

Forschungsarbeiten, die Interaktionen zwischen Parität und Zeit untersuchen, liegen derzeit nicht vor. Denkbar wäre, dass Mehrgebärende genau wissen, was auf sie zu kommt (unvorstellbare Schmerzen, an die eigenen Grenzen kommen, 
usw.). Sie sind sehr erleichtert, wenn die Geburt überstanden ist. Deshalb könnte die Angst nach der Geburt abnehmen. Bei Frauen, die bereits geboren haben, ist ein wichtiger Faktor zu beachten. Die Erfahrung, welche bei der vorausgegangenen Geburt gemacht wurde, hat einen gravierenden Einfluss auf die vor der Entbindung gemessene Angst. War die Geburt für die Frauen eine gute Erfahrung, lagen die Angstwerte signifikant tiefer als bei jenen, die die Niederkunft als etwas Negatives empfanden (Lunenfeld et al., 1984).

Erstgebärende können sich hingegen in der Regel nicht vorstellen, was Geburtsschmerzen sind und wie viel Kraft es braucht eine Geburt durchzustehen. Als Hebamme hört man von Erstgebärenden immer wieder, dass sie sich den Wehenschmerz nicht im entferntesten so heftig vorgestellt haben. Was die Umstellung nach der Geburt des Kindes für die Frau bedeutet, wird wohl auch sehr häufig von Erstgebärenden unterschätzt, was meine eigenen Erfahrungen zeigen. So liesse sich erklären, dass bei Erstgebärenden die Angst nach der Geburt eher ansteigt.

In vorliegender Diplomarbeit konnte gezeigt werden, dass sich Erst- und Mehrgebärende in bezug auf Trait- und auch State-Angst nicht signifikant unterschieden haben. Erstgebärende waren folglich nicht ängstlicher. Aus der Literatur ist hingegen bekannt, dass Erstgebärende ängstlicher sind.

Erickson (1975) kam zu dem Ergebnis, dass Erstgebärende mehr Angst haben als Mehrgebärende. Erhoben wurde von ihr allerdings nicht Trait- oder State-Angst, sondern Angst um sich selbst und um das Kind. Beide Angstformen waren bei Erstgebärenden signifikant höher als bei Mehrgebärenden. Somit ist ein Vergleich der Ergebnisse nur eingeschränkt möglich, zumal Untersuchungen aus den letzten Jahren nicht vor liegen.

Mögliche Erklärungen gibt es für beide Befunde. Einerseits kann man vermuten, dass das Wissen und die Erfahrung bezüglich Geburt (z.B. „es schon mal geschafft zu haben“; Wirkung von Schmerzmitteln unter der Geburt bereits einmal erfahren; unbeschreibliche Glücksgefühle, wenn das Baby in den eigenen Armen liegt usw.) Ängste nimmt. Andererseits könnte das Wissen um die z.B. so starken Schmerzen und Hilflosigkeit, Ängste erhöhen. Allerdings muss man bei solchen Vermutungen 
bedenken, dass das Erlebnis der vorausgegangenen Geburt eine wichtige Rolle bei der Modulation von Angst spielt (Lunenfeld et al., 1984). Bei den Erstgebärenden könnte auf der einen Seite Unwissenheit Unbefangenheit erzeugen, auf der anderen Seite allerdings auch zu Besorgnis führen. Aus der Literatur ist bekannt, dass eher letzteres zutreffend ist. Unwissenheit führt zu Ängsten. Lunenfeld et al. (1984) zeigten, dass Frauen, die sich über den Geburtsprozess schlecht informiert fühlten, vor der Geburt signifikant ängstlicher waren als jene, die sich gut informiert fühlten. Dies galt sowohl für Erstgebärende als auch für Mehrgebärende. Dieser Befund macht deutlich, wie wichtig geburtsvorbereitende Arbeit ist. Es kann also nicht eindeutig geklärt werden, ob sich Erstgebärende und Mehrgebärende bezüglich (vor der Geburt gemessene) Angst unterscheiden.

Zusammenfassend lässt sich sagen, dass sich die Frage, ob sich Angst nach einer Geburt verändert, nur beantwortet werden kann, wenn man viele Aspekte berücksichtigt. Sowohl die Parität als auch die Vorerfahrungen sowie das Wissen in bezug auf den Geburtsprozess spielen eine Rolle. Bei der Betrachtung von Unterschieden zwischen Erstgebärenden und Mehrgebärenden bezüglich Angst, müssen die Vorerfahrungen und der empfundene Informationsgrad unbedingt einbezogen werden.

Zur weiteren Klärung sind unbedingt Untersuchungen nötig, die sich mit diesem Zusammenhang auseinandersetzen und oben genannte Faktoren berücksichtigen.

\subsubsection{Interaktionen}

Der Datenlage nach zeigte sich eine signifikante Dreifach-Interaktion zwischen Trait-Angst, Bildung und Komplikationen. Die Angst nahm bei allen Frauen nach der Geburt ab, ausser bei den Frauen mit niedrigem Bildungsniveau und Komplikationen. Bei diesen Frauen wurde die Angst mehr. Es sieht so aus als wüssten die höher gebildeten Frauen genau, dass auch wenn die Betreuung der Geburt durch kompetentes Fachpersonal und mit den heutigen Möglichkeiten der Medizin erfolgt, Komplikationen auftreten können. Wenn die Geburt überstanden ist, fallen diese Sorgen weg, und die Angst wird weniger. Wenn Komplikationen auftreten, ist dies nicht ganz unerwartet und wirkt nicht nach. Frauen mit niedrigem Bildungsniveau scheinen an die Allmächtigkeit der heutigen Medizin zu 
glauben: wenn man sich in die Hände kompetenter Fachpersonen gibt, wird auch nichts passieren. Wenn trotzdem eine Komplikation auftritt, wird dieser Glaube zerstört und die Folge davon kann mehr Angst sein. Diese Frauen haben nun weniger Vertrauen in die Medizin. Es ist denkbar, dass sie sich Sorgen machen, wenn das Kind zum Beispiel erkrankt und die Medizin nicht im gewünschten Masse helfen kann.

Vergleichbare Befunde sind in der Literatur nicht zu finden, da bisher keine Interaktionen untersucht wurden.

Des weiteren zeigte sich ein signifikanter Haupteffekt bezüglich Bildung. Erstaunlicherweise hatten Frauen mit hohem Bildungsniveau sowohl höhere Stateals auch Trait-Angstwerte.

Aus der Literatur sind hingegen nur gegenteilige Ergebnisse bekannt. Helmbrecht (1974) stellte fest, dass die Häufigkeit von Schwangerschaftsängsten in Abhängigkeit von niedriger Schulbildung steigt. Lubin, Gardener \& Roth (1975) zeigten, dass die in der Schwangerschaft festgestellte Angst in Abhängigkeit von der Dauer des Schulbesuchs signifikant sinkt. Weniger gebildete Frauen erreichten höhere Angstwerte als Frauen mit höherer Bildung. Angst wurde mit verschiedenen schwangerschaftsunspezifischen Messinstrumenten (z.B. IPAT Anxiety Scale Questionnaire) erhoben, weshalb ein Vergleich mit vorliegendenen Ergebnissen besser möglich ist als mit dem Ergebnis von Helmbrecht (1975), der auschliesslich Schwangerschaftsängste erhoben hat.

Auch die Befunde von Rauchfuss (1996) legen nahe, dass Ängste mit dem Bildungsniveau zusammenhängen. Je höher die Bildung war, umso geringer war die Geburtsangst bei Frauen. Allerdings basiert dieser Befund auf Geburtsangst und nicht allgemeiner Angst wie in vorliegender Untersuchung, was einen Vergleich nur bedingt zulässt. Rauchfuss (1996) unterstreicht diesen Befund mit Ergebnissen von Forschern, die diese Beziehung ebenfalls zeigen konnten (z.B. Nowak, 1987; Köck et al., 1988; zit. nach Rauchfuss, 1996)

Denkbar ist, dass die unterschiedlichen Messinstrumente für die entegegengesetzten Befunde mitverantwortlich sind. Studien, die ebenfalls den STAI verwendeten, wurden nicht gefunden. 


\subsubsection{Prädiktoren}

\section{Vorhersage der Entbindungsform}

Das Bildungsniveau stellte sich als einziger Prädiktor für die Entbindungsform heraus (unter Konstanthaltung der anderen in die Regressionsanalyse eingegebenen Variablen). Ein hohes Bildungsniveau (Hochschulreife und Hochschulabschluss) der Frau, im Gegensatz zu niedrigerem Bildungsniveau (Mittlere Reife und Hauptschulabschluss), machte die Wahl für eine Hausgeburt wahrscheinlicher.

Dieser Befund entspricht dem der Studie von Kleiverda et al. (1989). Sie fanden noch weitere Prädiktoren für eine Hausgeburt: weniger konservative Einstellung gegenüber der weiblichen sozialen Rolle, relativ niedriges psychologisches Wohlbefinden und wenig Ängste, die die Geburt betreffen.

Aufgrund der Datenlage wurde die Familienstandskategorie „ledig“ als weiterer Prädiktor ermittelt (die anderen Variablen in der Regression wurden konstant gehalten). Wenn eine Frau unverheiratet war, im Gegensatz zu ein verheirateten oder geschiedenen Frau, war die Wahrscheinlichkeit für eine Hausgeburt grösser. Ähnliche Studien wurden nicht gefunden. Keiner der Forscher, die sich mit der Bestimmung von Prädiktorvariablen beschäftigten, analysierten den Einfluss des Familienstands. Somit könnte vorliegender Befund als erster Hinweis für diesen Zusammenhang gelten.

\section{Vorhersage von Komplikationen}

Durch die Parität liessen sich Komplikationen vorhersagen (wenn die anderen Variablen in der Regression konstant gehalten wurden). Je höher die Parität war, desto unwahrscheinlicher war das Auftreten von Komplikationen.

Ein vergleichbarer Befund ist bei Perkin et al. (1993) zu finden. Parität trug signifikant zur Varianzaufklärung bei. Die Parität war am stärksten mit allen in der Studie untersuchten Komplikationen verbunden. Alter war bei der Vorhersage von vaginal-operativen und operativen Entbindungen ein weiterer Prädiktor. In vorliegender Untersuchung war das Alter hingegen kein Prädiktor. Andere Variablen wie z.B. Angst, Depression und Körpergrösse spielten keine Rolle bei der 
Vorhersage von Komplikationen. Für Angst konnte dieses Ergebnis aufgrund der Datenlage dieser Diplomarbeit bestätigt werden. Ähnliche Befunde sind bei Beck et al. (1980) zu finden. Weder die Werte der vorgeburtlichen State-Angst noch die der Trait-Angst konnten das Auftreten von Komplikationen vorhersagen. In diesem Zusammenhang ergab sich auch ein signifikanter Unterschied zwischen Parität und Komplikationen. Frauen, die noch kein Kind geboren hatten, wiesen eine signifikant höhere Komplikationsrate auf als Mehrgebärende. Dieser Befund stimmt mit neuesten Forschungsergebnissen überein. Bai, Wong, Baumann \& Moshin (2002) kamen bei ihrer Studie zu dem Ergebnis (Alter, sozioökonomischer Status usw. wurden kontrolliert), dass Frauen, die noch kein Kind geboren hatten, ein signifikant höheres Risiko für das Auftreten von Komplikationen hatten als Frauen, die bereits ein oder mehr Kinder (1-3) geboren hatten. Wenn die Parität höher als drei war, wurde das Risiko für eine Komplikation wieder grösser.

\section{Vorhersage der State-Angst bei Geburtsbeginn}

Mit dem ersten Trait-Angstwert liess sich der State-Wert bei Geburtsbeginn voraussagen. Ausserdem stellte sich aufgrund der Datenlage heraus, dass die Entbindungsform ein Prädiktor für den State-Wert bei Geburtsbeginn ist. Bei Hausgeburtsfrauen, im Gegensatz zu Klinikgeburtsfrauen, lag der Wert um 6 Punkte tiefer. (Beides mal unter Konstanthaltung der anderen Variablen in der Regression).

Forschungsarbeiten, die sich mit der Untersuchung von Vorhersagen für die Angst bei Geburtsbeginn befassen, wurden nicht gefunden. So kann vorliegender Befund als erster Hinweis für einen Zusammenhang dieser Variablen gesehen werden.

Da sich nur wenige bzw. keine Studien der Bestimmung von Prädiktorvariablen für die Entbindungsform, Komplikationen und die Angst bei Geburtsbeginn widmen, bedarf es zur Klärung weitere Studien zu diesen Themen. 


\subsection{Stärken und Schwächen der Untersuchung}

Als positiv zu betrachten ist die Homogenität der Stichprobe, da so ein Vergleich beider Gruppen möglich war. Bezüglich Bildungsniveau und Familienstand unterschieden sich die Gruppen. Der Unterschied bezüglich des Bildungsstands scheint jedoch charakteristisch für die Gruppen zu sein.

Für die Erhebung der Daten konnte keine optimale Lösung gefunden werden, da es aus organisatorischen Gründen nicht möglich war, beide Gruppen in der selben Stadt zu befragen. Dass die Erhebung in mehreren Städten statt fand, begründet sich mit mehreren Tatsachen. In erster Linie war der vorhandene Kontakt zu Hebammen, Frauen und Krankenhaus ausschlaggebend für die Wahl der Städte. Es wäre aber auch nicht möglich gewesen, bei ca. 1\% Hausgeburten in ganz Deutschland, alle nötigen Hausgeburten an nur einem der Orte zu erheben. Da die Hausgeburtsfrauen und Klinikgeburtsfrauen (zumindest nicht zu gleichen Teilen) in verschiedenen Städten rekrutiert wurden, könnten Unterschiede zwischen den beiden Gruppen auch auf regionale Unterschiede zwischen den Städten beruhen (Walter, 1981). Beide Gruppen setzen sich jedoch aus städtischen sowie ländlichen Regionen zusammen, wodurch der diesbezügliche systematische Fehler so gering wie möglich gehalten wurde.

Als positiv ist zu werten, dass die Teilnehmerinnen der Studie nicht ausschliesslich in einer Klinik bzw. bei einer Hebamme rekrutiert worden sind. Diese Streuung verringerte den Klinik- bzw. Hebammeneffekt und führte zu einer besseren Repräsentativität der Stichprobe. Da der zeitliche und finanzielle Aufwand zu gross gewesen wäre, wurde auf die Vorteile einer Zufallsstichprobe verzichtet und die Nachteile einer zufälligen Stichprobe (z.B. Selektion, systematischer Fehler höher) in Kauf genommen. Aufgrund des quasi-experimentellen Designs, ist dieser Kritikpunkt aber nicht über zu bewerten.

In den 19 Monaten der Erhebung konnten 37 Hausgeburtsfrauen befragt werden. Für diesen Stichprobenumfang ist die niedrige Rate an Hausgeburten verantwortlich und, dass manche Frauen vor der zweiten Datenerhebung bereits geboren hatten. Somit konnten sie nicht mit in die Stichprobe aufgenommen 
werden. Mit einer noch grösseren Stichprobe könnten manche Ergebnisse signifikant werden, solange die Tendenz gleich bliebe.

Für weitere Untersuchungen ist es ratsam, eine grössere Stadt auszuwählen. Diese sollte eine ausreichend grosse Hausgeburtsrate aufweisen, damit beide Gruppen in einer Stadt rekrutiert werden können und eine Zufallsstichprobe gewonnen werden kann. Die Ergebnisse der Untersuchung mit einer solchen Stichprobe hätte eine bessere Aussagekraft bzw. Übertragbarkeit.

Von Vorteil ist das prospektive Längsschnittdesign. So konnten Veränderungen über die Zeit erfasst werden, die Messungen sind zudem reliabler als retrospektive Messungen.

Eine weitere Stärke dieser Studie ist die Erfassung von sowohl State-Angst als auch Trait-Angst. So ist eine differenzierte Betrachtung möglich. Die Erfassung von Angst erfolgte mit dem STAI, welches ein reliables und valides Instrument ist.

\subsection{Ausblick}

Die Ergebnisse bestätigen den Zusammenhang von Angst und Komplikationen nicht, mit der Ausnahme der Beziehung von Angst und speziellen Komplikationen. Die Sicherheit von Hausgeburten kann durch vorliegenden Befunde bestätigt werden. Es konnte kein Hinweis gefunden werden, dass sich Hausgeburtsfrauen und Klinikgeburtsfrauen bezüglich Angst unterschieden. Die Untersuchung konnte die Wichtigkeit angstreduzierender Arbeit unterstreichen.

Bereits im Rahmen der Diskussion der Ergebnisse sowie bei der Darstellung der Stärken und Schwächen dieser Untersuchung wurden einige Hinweise für zukünftige Studien gegeben und begründet. In diesem Abschnitt werden die wichtigsten noch einmal zusammengefasst und weitere Anregungen gegeben. 
Bei weiteren Untersuchungen ist es sinnvoll, eine noch grössere Stichprobe zu ziehen sowie eine Zufallsstichprobe auszuwählen. Dadurch würde sich die Repräsentativität der Stichprobe erhöhen. Dies könnte zur weiteren Aufklärung im Bereich „Angst im Zusammenhang von Schwangerschaft und Geburt", mit der Zusatzfrage „in Abhängigkeit von der Entbindungsform“ beitragen.

Bei der Forschung zum oben genannten Thema ist es sicherlich interessant, den Verlauf von Angst schon ab der Frühschwangerschaft bzw. vor der Konzeption zu beobachten, bevor (eventuell) die definitive Entscheidung für den Geburtsort gefallen ist. Mit einem prospektiven Längsschnittdesign könnten Veränderungen beobachtet werden, welche sich im Verlauf der ganzen Schwangerschaft und Geburt ergeben.

Sinnvoll ist ausserdem, den aus der Literatur (teilweise) bekannten Einfluss weiterer Faktoren wie z.B. Selbstwert, Qualität der Partnerschaft, Stress, usw. auf Komplikationen und Entbindungsform zu untersuchen, bzw. deren synergetische Wirkung.

Eine interessante Fragestellung für weitere Forschung ist, die Erhebung der Angst mit einem anderen Instrument durchzuführen. Speziell ein Instrument zur Messung von Geburtsangst (z.B. mit der G-A-S) erscheint als ein sehr interessantes Vorgehen. So können Unterschiede, bezüglich Messinstrument bzw. spezifisches Mass, erfasst werden.

Zudem ist sicherlich eine Untersuchung von Interesse, welche den Verlauf von Schwangerschaft und Geburt in Abhängigkeit von der Betreuungsperson (Hebamme oder Arzt, Hebamme gemeinsam mit Arzt) beobachtet. Es ist gut vorstellbar, dass die kontinuierliche Betreuung durch eine Hebamme einen angstreduzierenden Einfluss hat, welcher möglicherweise das Entstehen von Komplikationen moderiert. Hebammen können intensiver auf schwangerschaftsspezifische Probleme, Fragen und Bedürfnisse eingehen, als dies im Rahmen einer Vorsorgeuntersuchung in einer Arztpraxis (häufig hektischer 
Betrieb, mangelnde Zeit, usw.) möglich ist. Die Hebamme plant in der Regel eine Stunde für eine solche Untersuchung.

In einer Arztpraxis ist dies in der Regel so nicht möglich (Kosten!). Dadurch könnte zusätzlich die Akzeptanz von hebammenbetreuten Schwangerschaften und Geburten erhöht werden. 


\section{Zusammenfassung}

Die bisherige Forschung zum Zusammenhang zwischen Angst und Schwangerschafts- bzw. Geburtskomplikationen kam zu keinem einheitlichen Ergebnis.

Das Ziel der vorliegenden Arbeit war, den Zusammenhang zwischen Angst und Komplikationen zu präzisieren, indem Angst (präpartal und postpartal) und Komplikationen in einer prospektiven Längsschnittstudie untersucht wurden.

Ausserdem wurde untersucht, ob sich Haus- und Klinikgeburtsfrauen bezüglich oben genannter Variablen unterscheiden.

Die Stichprobe bestand aus jeweils 37 Frauen, die eine Haus- bzw. Klinikgeburt geplant hatten. Angst wurde gemäss dem State-Trait-Angstmodell verstanden (Spielberger et al. 1970) und wurde mit dem State-Trait-Angstinventar (deutsche Adaptation von Laux et al., 1981) erfasst. Die Erhebungen fanden präpartal in der letzten Schwangerschaftsphase, nach dem Modell von Gloger-Tippelt (1988), statt und nochmals einmalig postpartal. Es wurden jene Komplikationen erfasst, die in einschlägiger Literatur genannt werden (z.B. Pschyrembel \& Dudenhausen, 1991). Des weiteren wurden soziodemographische und schwangerschaftsspezifische Daten erhoben.

Es zeigten sich signifikante Unterschiede bei den soziodemographischen Daten zwischen den Hausgeburtsfrauen und Klinikgeburtsfrauen. Hausgeburtsfrauen hatten ein signifikant höheres Bildungsniveau und waren signifikant häufiger ledig. Hypothese $1 \mathrm{a}$, mit der man untersuchen wollte, ob sich Frauen mit und ohne Schwangerschaftskomplikationen in bezug auf Angst unterscheiden, konnte aufgrund der Datenlage nicht untersucht werden.

Frauen mit und ohne Geburtskomplikationen unterschieden sich bezüglich der gemessenen Angst nicht signifikant, weshalb die Hypothese $1 \mathrm{~b}$ abgelehnt wurde. Hypothese 2 ( $\mathrm{H} 0)$ bewährte sich, es wurde kein signifikanter Unterschied zwischen den Gruppen bei der Komplikationsrate gefunden. Allerdings wurden signifikante 
Unterschiede festgestellt, wenn die Komplikationen kategorial in die Analysen eingingen. Komplikationen in der Nachgeburtsperiode und Mehrfachkomplikationen traten signifikant häufiger in der Klinikgeburtsgruppe auf.

Hypothese 3 konnte nicht bestätigt werden, da sich kein signifikanter Unterschied zwischen den Hausgeburts- und Klinikgeburtsfrauen in bezug auf Angst zeigte. Im Verlauf der State-Angst ergaben sich signifikante Unterschiede zwischen den Messungen. Bezüglich des Bildungsstands ergaben sich signifikante Unterschiede bei den State- und Trait-Angstwerten.

Des weiteren konnten Prädiktoren für Komplikationen, Entbindungsform und Angst bei Geburtsbeginn ermittelt werden.

Der signifikante Unterschied bezüglich des Bildungsniveaus zwischen den Gruppen war mit einem aus der Literatur bekannten Befund übereinstimmend und scheint ein typisches Merkmal der untersuchten Gruppen zu sein. Für den Unterschied bezüglich des Familienstands konnte nur ein gegenteiliger Befund in der Literatur gefunden werden.

Für die Ergebnisse der Hypothesenprüfungen fanden sich vergleichbare Befunde aus der Literatur.

Für die Unterschiede im Verlauf der State-Angst waren (teilweise) übereinstimmende Ergebnisse aus der Forschung zu finden. Die Unterschiede zwischen den Bildungsniveaus widersprachen den Befunden aus der Literatur.

Einige der gefundenen Prädiktorvariablen stehen im Einklang mit Befunden aus der Literatur, andere konnten nicht verglichen werden, da derartige Untersuchungen fehlen.

Für weitere Untersuchungen wäre es interessant, eine grössere Stichprobe bereits in der Frühschwangerschaft oder sogar vor der Konzeption zu untersuchen. Zusätzlich sollten noch weitere Variablen wie z.B. Selbstwert oder Qualität der Partnerschaft miteinbezogen werden. 


\section{Literaturverzeichnis}

Ackermann-Liebrich, U., Voegeli, T., Gunter-Witt, K., Kunz, I., Zullig, M., Schindler, C. \& Maurer, M. (1996). Home versus hospital deliveries: follow up study of matched pairs for procedures and outcome. British Medical Journal, 313 (7068), 1313-1318.

Asendorpf, J.B. (1996). Psychologie der Persönlichkeit: Grundlagen. Berlin: Springer.

Ashbury, J. (1980). The crisis of childbirth: can information and childbirth education help? Journal of Psychosomatic Research, 24, 9-13.

Badinter, E. (1980). Die Mutterliebe. Geschichte eines Gefühls vom 17. Jahrhundert bis heute. München, Zürich: R. Piper \& Co.

Baltes, P.B. (1990). Entwicklungspsychologie der Lebensspanne: Theoretische Leitsätze. Psychologische Rundschau, 41, 1-24.

Bai, J., Wong, F.W., Baumann, A. \& Moshin, M. (2002). Parity and pregnancy outcomes. American Journal of Obstetrics \& Gynecology, 186 (2), 274-278.

Barlow, D.H. (1988). Anxiety and its disorders. New York: Guilford Press.

Bayer, R. \& Hoff, F. (1959). Modellversuche zur Darstellung der durch Angst und Schmerz ausgelösten Erregungsübertragung auf den nicht graviden, graviden und gebärenden menschlichen Uterus. Zeitschrift für Geburtshilfe und Gynäkologie, 153, 105-112.

Beck, N.C., Siegel, L.J., Davidson, N.P., Kormeier, S., Breitenstein, A. \& Hall, D.G. (1980). The prediction of pregnancy outcome: maternal preparation, anxiety and attitudinal sets. Journal of Psychosomatic Research, 24, 343-351.

Beckmann, D. (1979). Geschlechtsrollen und Paardynamik. In: H. Pross (Hrsg.), Familie wohin? Leistungen, Leistungsdefizite und Leistungswandlungen der Familie in hochindustrialisierten Gesellschaften, 169-195. Hamburg: Rowohlt.

Berninghausen, J. (1980). Der Traum vom Kind-Geburt eines Klischees. Mutterschaft: Ideologie, Wunsch und Wirklichkeit. Frankfurt am Main: Ullstein. 
Bortz, J. (1999). Statistik für Sozialwissenschaftler. Berlin: Springer.

Brähler, C. (1990). Familie, Kinderwunsch, Unfruchtbarkeit. Opladen:

Westdeutscher Verlag.

Bund Deutscher Hebammen e.V., Bund freiberuflicher Hebammen Deutschland e.V.

\& Netzwerk zur Förderung der Idee der Geburtshäuser in Deutschland e.V.

(2000). Hebammengeleitete Geburtshilfe. Empfehlungen und

Auswahlkriterien des Geburtsortes.

Bundesausschuss der Ärzte und Krankenkassen (1997). Mutterpass.

Bundesminister für Familie, Senioren, Frauen und Jugend (1998). Die Pluralität von

Lebensformen und Lebenslagen. Kinder und ihre Kindheit in Deutschland.

Eine Politik für Kinder im Kontext von Familienpolitik, 127-142. Stuttgart:

Kohlhammer.

Cattel, R.B. \& Scheier, I.H. (1961). The meaning and measurement of neuroticism and anxiety. New York: Ronald Press.

Chatterjee; S. \& Hadi, A.S. (1988). Sensitivity Analysis in Linear Regression. New York: John Wiley \& Sons.

Crandon, A.J. (1979). Maternal anxiety and obstetric complications. Journal of Psychosomatic Research, 23, 109-111.

Davids, A. \& DeVault, S. (1962). Maternal anxiety during pregnancy and childbirth abnormalities. Psychosomatic Medicine, 24, 464-470.

Davids, A., DeVault, S. \& Talmadge, M. (1961). Anxiety, pregnancy, and childbirth abnormalities. Journal of Consulting Psychology, 25, 74-77.

Davies-Osterkamp, S. \& Beckmann, D. (1982). In D. Beckmann,

S. Davies-Osterkamp \& J.-W. Scheer (Hrsg.), Medizinische Psychologie:

Forschung für Klinik und Praxis, 493-513. Berlin: Springer.

Dick-Read, G. (1972). Mutterwerden ohne Schmerz. Hamburg: Hoffmann \& Campe.

Dyer, E.D. (1963). Parenthood as crisis: A re-study. Marriage and Family Living, 25, 196-201.

Edward, K.R. \& Jones, M.R. (1970). Personality changes related to pregnancy and obstetric complications. Proceedings of the 78th Annual Convention of the American Psychological Association, 5, 341-342. 
Erickson, M.T. (1975). The influence of health factors on psychological variables predicting complications of pregnancy, labor and delivery. Journal of Psychosomatic Research, 20, 21-24.

Erikson, E.H. (1966). Wachstum und Krisen der gesunden Persönlichkeit. In E.H. Erikson (Hrsg.), Identität und Lebenszyklus, 55-122. Frankfurt: Suhrkamp. EWIM (European Workgroup of Independent Midwives). Texte zur Maieutik: Betreuung während einer normalen Geburt. Unna: Phoenix Shanti.

Falorni, M.L., Fornasarig, A. \& Stefanile, C. (1979). Research about anxiety effects on the pregnant woman and her newborn child. In L. Carenza \& L. Zichella (Hrsg.), Emotions and reproducting. Proceedings of the Serono Symposium, 1147-1153. London: Academic Press.

Filipp, S.H. (1981). Kritische Lebensereignisse. München: Urban \& Schwarzenberg. Flor, H. \& Hermann, C. (1999). Schmerz. In: H. Flor, N. Birbaumer \& K. Hahlweg (Hrsg.), Enzyklopädie der Psychologie: Grundlagen der Verhaltensmedizin, 249-305. Göttingen: Hogrefe.

Gesellschaft für Qualität in der ausserklinischen Geburtshilfe e.V. (2001).

Qualitätsberichte 1999-2001: Ausserklinische Geburtshilfe in Deutschland. Bonn: Rigotti-Klarhorst.

Gloger-Tippelt, G. (1985). Der Übergang zur Elternschaft. Eine entwicklungspsychologische Analyse. Zeitschrift für Entwicklungspsychologie und Pädagogische Psychologie, 17, 53-92.

Gloger-Tippelt, G. (1988). Schwangerschaft und erste Geburt: Psychologische Veränderungen der Eltern. Stuttgart: Kohlhammer.

Gorsuch, R.L. \& Key, M.K. (1974). Abnormalities of Pregnancy as a Function of Anxiety and Life Stress. Psychosomatic Medicine, 36 (4), 352-362.

Gulbransen, G., Hilton, J., McKay, L. \& Cox, A. (1997). Home births in New Zealand: 1973-93 incidence and mortality. New Zealand Medical Journal, 110, 87-89.

Guttman, D. (1975). Parenthood: A key to the comparative study of the life cycle. In N. Datan \& L.H. Ginsberg (Hrsg.), Life-span development psychology, 167-184. New York: Academic Press. 
Hauffe, U. (1987). Ansprüche an geburtsvorbereitende Arbeit. In P.G. FedorFreybergh (Hrsg.), Pränatale und perinatale Psychologie und Medizin: Begegnung mit dem Ungeborenen, 313-320. Älvsjö: Saphir.

Helmbrecht, H. (1975). Beziehungen zwischen Vorstellungen und Verhaltensweisen in der Schwangerschaft einerseits und sozialen Merkmale andererseits.

Unveröffentlichte Dissertation. Erlangen-Nürnberg:

Friedrich-Alexander-Universität.

Hill, R. (1949). Families under Stress. New York: Harper.

Hobbs, D.F. (1965). Parenthood as crisis: A third study. Journal of Marriage and the Family, 27, 367-372.

Huber, O. (1995). Das psychologische Experiment: Eine Einführung. Bern: Hans Huber.

Hüter, K.A. (1966). Die medikamentöse und psychosomatische Geburtsleitung. Vergleichende biometrische Untersuchungen. Basel: Karger.

Istvan, J: (1986). Stress, Anxiety, and Birth Outcome: A Critical Review of the Evidence. Psychological Bulletin, 100 (3), 331-348.

Janssen, P.A., Lee, S.K., Ryan, E.M., Etches, D.J., Farquharson, D.F., Peacock, D. \& Klein, M.C. (2002). Outcome of planned home births. Canadian Medical Association Journal, 166 (3), 315-322.

Kapp, F.T., Hornstein, S. \& Graham, V.T. (1963). Some psychologic factors in prolonged labor due to inefficient uterine action. Comprehensive Psychiatry, 4, 9-18.

Kelly, J.V. (1962). Effect of fear upon uterine mobility. American Journal of Obstetrics and Gynecology, 83, 576-581.

Kelm-Kahl, I. (1990). Hausgeburt. Reinbek bei Hamburg: Rowohlt Taschenbuch. Kleiverda, G., Steen, A.M., Andersen, I., Treffers, P.E. \& Everaerd, W. (1990). Place of delivery in The Netherlands: maternal motives and background variables related to preference for home or hospital confinement. European Journal of Obstetrics \& Gynaecology and Reproductive Biology, 36, 1-9.

Krohne, H.W. (1996). Angst und Angstbewältigung. Stuttgart: Kohlhammer. Künzel, W. (1995). Epidemiologie der Frühgeburt. Der Gynäkologe, 28 (3), 130135. 
Larbig, W. \& Birbaumer, N. (1986). Psychophysiologie der Angst. In V. Faust

(Hrsg.), Compendium Psychiatricum. Angst-Furcht-Panik, 16-26.

Stuttgart: Hippokrates.

Laux, L., Glanzmann, P., Schaffner, P. \& Spielberger C.D. (1981). Das State-TraitAngstinventar. Theoretische Grundlagen und Handanweisung. Weinheim: Beltz Testgesellschaft.

LeMasters, E.E. (1957). Parenthood as crisis. Marriage an Family Living, 19, 352355.

Levitt, E.E. (1971). Die Psychologie der Angst. Stuttgart: Kohlhammer.

Linder, R. (1994). Haus- und Praxisgeburten: Dokumentation der 1. Tagung für Haus- und Praxisgeburtshilfe. Frankfurt: Mabuse.

Lubin, B., Gardener, S.H. \& Roth, A. (1975). Mood and somatic symptoms during pregnancy. Psychosomatic Medicine, 37 (2), 136-146.

Lukesch, H. \& Lukesch, M. (1976). S-S-G. Ein Fragebogen zur Messung von Einstellungen zu Schwangerschaft, Sexualität und Geburt. Göttingen: Hogrefe.

Lukesch, H. (1981). Schwangerschafts- und Geburtsängste. Stuttgart: Enke.

Lukesch, H. (1982). Schwangerschaftseinstellung und Geburtsverlauf: Die Bedeutung psychischer Faktoren für Schwangerschaftsverlauf, Geburt und Kindesentwicklung. In S. Schindler (Hrsg.), Geburt: Eintritt in eine neue Welt, 65-74. Göttingen: Hogrefe.

Lukesch, H. (1983). Geburts-Angst-Skala. Handanweisung. Göttingen: Hogrefe. Lunenfeld, E., Rosenthal, J., Larholt, K.M. \& Insler, V. (1984). Childbirth experience - psychological, cultural and medical associations. Journal of Psychosomatic Obstetrics and Gynaecology, 3, 165-171.

Macfarlane, A. (1972). Die Geburt. Stuttgart: Klett-Cotta.

Macfarlane, A., McCandlish, R. \& Campbell, R. (2000). Choosing between home and hospital delivery. British Medical Journal, 320 (7237), 798-799.

Martius, G. (1990). Hebammenlehrbuch. Stuttgart: Georg Thieme.

McDonald, R.L. \& Christakos, H.C. (1963). Relationship of emotional adjustment during pregnancy to obstetric complications. American Journal of Obstetric Gynecology, 86, 341-348. 
McDonald, R.L., Gynther, M.D. \& Christakos, A.C. (1963). Relation between maternal anxiety and obstetric complications. Psychosomatic Medicine, 25, 357-363.

McDonald, R.L. \& Parham, K.J. (1964). Relations of emotional changes during pregnancy to obstetric complications in unmarried primigravidae. American Journal of Obstetric Gynecology, 90, 195-201.

McDonald, R.L. (1965). Personality Characteristics in Patients with Three Obstetric Complications. Psychosomatic Medicine, 27 (4), 383-390.

McDonald, R.L. (1968). The role of emotional factors in obstetric complications: A review. Psychosomatic Medicine, 30, 222-237.

Melamed, B.G. \& Siegel, L.J. (1980). Lehrbuch der Verhaltensmedizin. Stuttgart: Kohlhammer

Molfese, V.J.; Bricker, M.C., Manion, L.G., Beadnell, B., Yaple, K. \& Moires, K.A. (1987). Anxiety, depression and stress in pregnancy: a multivariate model of intra-partum risks and pregnancy outcomes. Journal of Psychosomatic Obstetrics and Gynaecology, 7, 77-92.

Molinski, H. (1975). Geburtshilfliche Symptomatik als Folge gestörten Geburtsverhaltens. Zeitschrift für Geburtshilfe und Perinatologie, 179, 383387.

Morcos, F.H. \& Funke-Ferber, J. (1979). Anxiety an depression in the mother and father and their relationship to physical complications of pregnancy and labour. In L. Carenza \& L. Zichella (Hrsg.), Emotion and reproduction, Proceedings of the Serono Symposium, 757-765. London: Academic Press.

Neumann, H. (1987). Ziel und Sinn einer Geburtsvorbereitung. In P.G. FedorFreybergh (Hrsg.), Pränatale und perinatale Psychologie und Medizin: Begegnung mit dem Ungeborenen, 375-378. Älvsjö: Saphir.

Norbeck, J.S. \& Tilden, V.P. (1983). Life stress, social support, and emotional disequilibrium in complications of pregnancy: A prospective, multivariate design. Journal of Health and Social Behavior, 24, 30-46.

Pinel, J.P.J. (2002). Biopsychology. Boston: Allyn \& Bacon. 
Rauchfuss, M. (1996). Psychosomatische Aspekte von

Schwangerschaftskomplikationen. In M. Rauchfuss, A. Kuhlmey \& P.

Rosemeier (Hrsg.), Frauen in Gesundheit und Krankheit: Die neue frauenheilkundliche Perspektive, 65-96. Berlin: Trafo.

Richter, D. \& Stauber, M. (1990). Gynäkologie und Geburtshilfe. In T. von Uexküll (Hrsg.), Psychosomatische Medizin, 941-974. München: Urban \& Schwarzenberg.

Rizzardo, R., Magni, G., Cremonese, C., Talamo Rossi, R. \& Cosentino, M. (1988).

Variations in Anxiety Levels during Pregnancy and Psychosocial Factors in

Relation to Obstetric Complications. Journal of Psychotherapy \&

Psychosomatics , 49, 10-16.

Perkin, M.R., Bland, J.M., Peacock, J.L. \& Anderson, H.R. (1993). The effect of anxiety and depression during pregnancy on obstetric complications. British Journal of Obstetrics \& Gynaecology, 100 (7), 629-634.

Ryding, E.L., Wijma, B., Wijma, K. \& Rydhstrom, H. (1998). Fear of childbirth during pregnancy may increase the risk of emergency cesarean section. Acta Obstetrica et Gynecologica Scandinavica, 77, 542-547.

Pschyrembel, W. (1990). Pschyrembel Klinisches Wörterbuch. Berlin: de Gruyter.

Pschyrembel, W. \& Dudenhausen, J.W. (1991). Praktische Geburtshilfe mit geburtshilflichen Operationen. Berlin: de Gruyter.

Sartory, G. (1997). Angst-Störungen: Theorien, Befunde, Diagnostik und

Behandlung. Darmstadt: Wissenschaftliche Buchgesellschaft.

Sartory, G. \& Diener, H.-C. (2001). Kopfschmerz und Migräne. In H. Flor, N.

Birbaumer \& K. Hahlweg (Hrsg.), Enzyklopädie der Psychologie:

Anwendungen der Verhaltensmedizin, 1-34. Göttingen: Hogrefe.

Spielberger, C.D., Gorsuch, R.L. \& Lushene, R.E. (1970). Manual for the State-Trait Anxiety Inventory. Palo Alto CA: Consulting Psychologists Press.

Spielberger, C.D. (1972). Anxiety as an emotional state. In C.D. Spielberger (Hrsg.), Anxiety: Current trends in theory and research. Band 1. New York: Academic Press.

Stadelmann, I. (1994). Die Hebammen-Sprechstunde. Kempten: Ingeborg Stadelmann Eigenverlag. 
Stähler, E. \& Stähler, H. (1983). In S. Schindler \& H. Zimprich (Hrsg.), Ökologie der Perinatalzeit, 89-95. Stuttgart: Hippokrates.

Treffers, P.E. \& Laan, R. (1986). Regional perinatal mortality and regional hospitalization at delivery in The Netherlands, 93, 690-693. European Journal of Obstetrics, Gynaecology \& Reproductive Biology.

Treffers, P.E. (1990). Home births and minimal medical interventions. Journal of the American Medical Association, 264, 2203, 2207-2208.

Walter, H. (1981). Region und Sozialisation. Stuttgart: Frommann-Holzboog.

Wiegers, T.A., Keirse, M.J., van der Zee, J. \& Berghs, G.A. (1996). Outcome of planned home and planned hospital births in low risk pregnancies:

prospective study in midwifery practices in The Netherlands. British Medical Journal, 313 (7068), 1309-1313.

Windsor-Oettel, V. (1992). Angst und Selbstwert von Frauen vor und nach der Entbindung in Abhängigkeit von der Entbindungsform. Frankfurt am Main: Lang.

Wimmer-Puchinger, B. (1992). Schwangerschaft als Krise: Psychosoziale Bedingungen von Schwangerschaftskomplikationen. Berlin: Springer.

Zimmermann, D. (1998). Geburtshäuser: Ganzheitliche Geburt als Alternative. München: C.H. Beck. 


\section{Anhang}




\section{Anhang A: Explorative Analysen}

Tabelle 25: Korrelationsmatrix

\begin{tabular}{l|c|c|c|c|c|c} 
& STAI X1,1 & STAI X1,2 & STAI X1,3 & STAI X1,4 & STAI X2,1 & STAI X2,2 \\
\hline STAI X1,1 & 1 &, $536^{* *}$ &, $382^{* *}$ &, 222 &, $727^{* *}$ &, $301^{*}$ \\
\hline STAI X1,2 &, $536^{* *}$ & 1 &, $463^{* *}$ &, 216 &, $51^{* *}$ &, $286^{*}$ \\
\hline STAI X1,3 &, $382^{\star *}$ &, $463^{\star *}$ & 1 &, 190 &, $446^{* *}$ &, $315^{\star *}$ \\
\hline STAI X1,4 &, 222 &, 216 &, 190 & 1 &, $258^{\star}$ &, $716^{* *}$ \\
\hline STAI X2,1 &, $727^{* *}$ &, $51^{* *}$ &, $446^{* *}$ &, $258^{*}$ & 1 &, $532^{* *}$ \\
\hline STAI X2,2 &, $301^{*}$ &, $286^{*}$ &, $315^{* *}$ &, $716^{* *}$ &, $532^{* *}$ & 1 \\
\hline
\end{tabular}

${ }^{*}$ Die Korrelation nach Pearson ist auf dem Niveau von 0,01 (2-seitig) signifikant.

* Die Korrelation nach Pearson ist auf dem Niveau von 0,05 (2-seitig) signifikant.

Tabelle 26: Bildung und Komplikation ( $\chi_{-}-$Test)

\begin{tabular}{l|c|c|c} 
Komplikationen & $\begin{array}{c}\text { Hauptschulabschluss/ } \\
\text { Mittlere Reife }\end{array}$ & $\begin{array}{c}\text { Fachhoch-/ } \\
\text { Hochschulreife }\end{array}$ & $\begin{array}{c}\text { Fachhoch-/ } \\
\text { Hochschulabschluss }\end{array}$ \\
\hline nein & $23(47,9 \%)$ & $13(72,2 \%)$ & $12(70,6 \%)$ \\
\hline ja & $16(41,0 \%)$ & $5(27,8 \%)$ & $5(39,4 \%)$ \\
\hline
\end{tabular}

$\chi_{-}(\mathrm{LR})=1,276+; \mathrm{df}=2$

$+p>0,05$

Tabelle 27: Alter und Kompliaktionen ( $\chi_{-}$-Test)

\begin{tabular}{l|c|c} 
Komplikationen & 20-30 Jahre & über 30 Jahre \\
\hline nein & $19(36,6 \%)$ & $29(60,4)$ \\
\hline ja & $13(50,0 \%)$ & $13(50,0 \%)$ \\
\hline
\end{tabular}

$\chi_{-}(\mathrm{LR})=0,743+; \mathrm{df}=1$;

$+\mathrm{p}>0,05$ 
Tabelle 28: Alter und State-/Trait-Angst (zweifaktorielle Varianzanalyse mit Messwiederholung)

\begin{tabular}{l|c|c|c|c|c} 
Effekt & Wilks Lambda & $\mathrm{F}$ & $\mathrm{df}_{1}$ & $\mathrm{df}_{2}$ & Signifikanz \\
\hline State-Angst ${ }^{*}$ Alter & 0,988 & 0,274 & 3 & 65 & 0,844 \\
\hline Trait-Angst ${ }^{*}$ Alter & & 0,412 & 1 & 69 & 0,523 \\
\hline
\end{tabular}

Tabelle 29: Familienstand und Komplikationen ( $\chi_{-}$-Test)

\begin{tabular}{l|c|c} 
Komplikationen & verheiratet/geschieden & ledig \\
\hline nein & $34(75,6 \%)$ & $11(24,4)$ \\
\hline ja & $20(80,0 \%)$ & $5(20,0 \%)$ \\
\hline
\end{tabular}

$\chi_{-}(\mathrm{LR})=0,183+; \mathrm{df}=1$

$+p>0,05$

Tabelle 30: Familienstand und State-/Trait-Angst (zweifaktorielle Varianzanalyse mit Messwiederholung)

\begin{tabular}{l|c|c|c|c|c} 
Effekt & Wilks Lambda & $\mathrm{F}$ & $\mathrm{df}_{1}$ & $\mathrm{df}_{2}$ & Signifikanz \\
\hline State-Angst ${ }^{*}$ Familienstand & 0,937 & 1,370 & 3 & 61 & 0,262 \\
\hline Trait-Angst ${ }^{\star}$ Familienstand & -- & 2,232 & 1 & 65 & 0,140 \\
\hline
\end{tabular}

Box-Test bei Trait-Analyse signifikant: $F(3,11862)=3,233 ; p<0,05$

Tabelle 31: Fehlgeburten und State-/Trait-Angst (zweifaktorielle Varianzanalyse mit Messwiederholung)

\begin{tabular}{l|c|c|c|c|c} 
Effekt & Wilks Lambda & $\mathrm{F}$ & $\mathrm{df}_{1}$ & $\mathrm{df}_{2}$ & Signifikanz \\
\hline State-Angst ${ }^{\star}$ Fehlgeburten & 0,948 & 1,194 & 3 & 65 & 0,319 \\
\hline Trait-Angst ${ }^{\star}$ Fehlgeburten & -- & 3,071 & 1 & 69 & 0,084 \\
\hline
\end{tabular}


Tabelle 32: State-/Trait-Angst und Komplikationskategorie 1 (zweifaktorielle Varianzanalyse mit Messwiederholung)

\begin{tabular}{l|c|c|c|c|c} 
Effekt & Wilks Lambda & $\mathrm{F}$ & $\mathrm{df}_{1}$ & $\mathrm{df}_{2}$ & Signifikanz \\
\hline State-Angst ${ }^{\star}$ Kompl.kat. 1 & 0,986 & 0,316 & 3 & 65 & 0,814 \\
\hline Trait-Angst*Kompl.kat. 1 & -- & 0,262 & 1 & 69 & 0,619 \\
\hline
\end{tabular}

Box-Test bei State Analyse signifikant: $F(10,4754)=2,657 ; p<0,05$

Tabelle 33: State-/Trait-Angst und Komplikationskategorie 2 (zweifaktorielle Varianzanalyse mit Messwiederholung)

\begin{tabular}{l|c|c|c|c|c} 
Effekt & Wilks Lambda & $\mathrm{F}$ & $\mathrm{df}_{1}$ & $\mathrm{df}_{2}$ & Signifikanz \\
\hline State-Angst ${ }^{\star}$ Kompl.kat. 2 & 0,989 & 0,233 & 3 & 65 & 0,873 \\
\hline Trait-Angst ${ }^{\star}$ Kompl.kat. 2 & -- & $<0,001$ & 1 & 69 & 0,988 \\
\hline
\end{tabular}

Tabelle 34: State-/Trait-Angst und Mehrfachkomplikationen (zweifaktorielle Varianzanalyse mit Messwiederholung)

\begin{tabular}{l|c|c|c|c} 
Effekt & $\mathrm{F}$ & $\mathrm{df}_{1}$ & $\mathrm{df}_{2}$ & Signifikanz \\
\hline State-Angst ${ }^{\star}$ Mehrfachkompl. & 0,339 & 3 & 63 & 0,797 \\
\hline Trait-Angst ${ }^{*}$ Mehrfachkompl. & 0,099 & 1 & 23 & 0,756 \\
\hline
\end{tabular}

Mauchly-Test nicht signifikant: Mauchly $\mathrm{W}=0,862 ; \mathrm{df}=5 ; \mathrm{p}>0,05$.

Tabelle 35: Komplikationen Kategorie 1 und Entbindungsform ( $\chi_{-}$-Test)

\begin{tabular}{l|c|c} 
Komplikationen (Kategorie 1) & Klinikgeburt & Hausgeburt \\
\hline nein & $26(70.3 \%)$ & $28(75,7 \%)$ \\
\hline ja & $11(29,70 \%)$ & $9(24,3 \%)$ \\
\hline$\chi_{-}(\mathrm{LR})=0,274+; \mathrm{df}=1$ & \\
$+p>0,05$
\end{tabular}


Tabelle 36: Komplikationen Kategorie 3 und Entbindungsform (Fisher's Exact Test)

\begin{tabular}{l|c|c} 
Komplikationen (Kategorie 3) & Klinikgeburt & Hausgeburt \\
\hline nein & $32(85,5 \%)$ & $35(94,6 \%)$ \\
\hline ja & $5(13,5 \%)$ & $2(5,4 \%)$ \\
\hline
\end{tabular}

$\chi_{-}(\mathrm{LR})=1,463+; \mathrm{df}=1$

$+p>0,05$

Tabelle 37: Komplikationen Kategorie 1 und Entbindungsform bei Erstgebärenden (Fisher's Exact Test)

\begin{tabular}{l|c|c} 
Komplikationen (Kategorie 1) & Klinikgeburt & Hausgeburt \\
\hline nein & $13(65,0 \%)$ & $6(54,5 \%)$ \\
\hline ja & $7(35,0 \%)$ & $5(45,5 \%)$ \\
\hline$\chi_{-}(\mathrm{LR})=0,325+; \mathrm{df}=1$ & \\
$+p>0,05$
\end{tabular}

Tabelle 38: Komplikationen Kategorie 1 und Entbindungsform bei Mehrgebärenden (Fisher's Exact Test)

\begin{tabular}{l|c|c} 
Komplikationen (Kategorie 1) & Klinikgeburt & Hausgeburt \\
\hline nein & $13(76,5 \%)$ & $22(84,6 \%)$ \\
\hline ja & $4(23,5 \%)$ & $4(15,4 \%)$ \\
\hline$\chi_{-}(\mathrm{LR})=0,443+; \mathrm{df}=1$ & \\
$+p>0,05$
\end{tabular}

Tabelle 39: Komplikationen Kategorie 2 und Entbindungsform bei Erstgebärenden (Fisher's Exact Test)

\begin{tabular}{l|c|c} 
Komplikationen (Kategorie 2) & Klinikgeburt & Hausgeburt \\
\hline nein & $13(65,0 \%)$ & $11(100 \%)$ \\
\hline
\end{tabular}




\begin{tabular}{l|c|c}
\hline ja & $7(35,0 \%)$ & 0 \\
\hline$\chi \quad(L R)=7,220^{*} ; \mathrm{df}=1$
\end{tabular}

${ }^{*} \mathrm{p}<0,05$

Tabelle 40: Komplikationen Kategorie 2 und Entbindungsform bei Mehrgebärenden

(Fisher's Exact Test)

\begin{tabular}{l|c|c} 
Komplikationen (Kategorie 2) & Klinikgeburt & Hausgeburt \\
\hline nein & $13(76,5 \%)$ & $26(100 \%)$ \\
\hline ja & $4(23,5 \%)$ & 0 \\
\hline
\end{tabular}

$\chi_{-}(\mathrm{LR})=8,065^{\star} ; \mathrm{df}=1$

${ }^{*} p<0,05$

Tabelle 41: Prüfung auf Interaktionen (vierfaktorielle Varianzanalyse mit Messwiederholung)

\begin{tabular}{l|c|c|c|c} 
Effekt & $\mathrm{F}$ & $\mathrm{df}_{1}$ & $\mathrm{df}_{2}$ & Signifikanz \\
\hline Zeit $^{\star}$ Bildung ${ }^{\star}$ Entbindungsform & 0,527 & 1 & 63 & 0,471 \\
\hline Zeit $^{\star}$ Komplik. ${ }^{\star}$ Entbindungsform & 0,146 & 1 & 63 & 0,704 \\
\hline Zeit $^{\star}$ Bildung ${ }^{\star}$ Kompl.. ${ }^{\star}$ Entb.form & 1,096 & 1 & 63 & 0,299 \\
\hline
\end{tabular}


Anhang B: Verwendeter Fragebogen 